\title{
2011 CAEP/ACMU Scientific Abstracts June 5-9, 2011 St. John's, NL
}

\begin{abstract}
Disclaimer: The large number of abstracts submitted and the short time interval between submission and publication does not permit communication with authors, abstract revision or CJEM editorial review. The following abstracts are presented, unedited, as they were submitted to the CAEP Research Committee. Abstract authors are from the department or division of emergency medicine of their respective universities unless otherwise specified.
\end{abstract}

Avertissement : Le grand nombre de résumé soumis et le court délai entre leur réception et la date de publications on empêché la communication avec les auteurs, la révision des résumés, ou l'évaluation par le comité du réduction du JCMU. Les résumés qui suivent sont présentés non édités, tel qu'ils ont été soumis au Comité de Recherche de I'ACMU. Les auteurs des résumés sont rattachés au département de médecine d'urgence de leur université respective, sauf indication contraire.

\section{Oral presentations}

\section{Vaillancourt, Christian}

cvaillancourt@ohri.ca

FACTORS influencing the intentions of nurses and respiratory therapists to use automated external defibrillators during in-hospital cardiac arrests: an institutional survey

Christian Vaillancourt, Ann Kasaboski, Jessica Tweedle, Jan L. Jensen, Manya Charette, Jeremy Grimshaw, Jamie Brehaut, Martin H. Osmond, George A. Wells, Ian G. Stiell; Department of Emergency Medicine, University of Ottawa, Ottawa, ON

Introduction: Most in-hospital defibrillators are equipped with an automated external defibrillator (AED) mode, yet nurses and respiratory therapists (RT) trained in their use are seldom authorized to use them. We sought to determine factors associated with the successful implementation of a in-hospital AED program for use by nurses and RT during in-hospital cardiac arrest. Methods: We conducted this paper-based survey among nurses and RTs purposefully recruited from various clinical programs at three teaching hospital campuses using multiple distribution attempts and a modified Dillman method. We developed the interview guide based on the constructs of the Theory of Planned Behavior (TPB), which elicits salient attitudes, social influences, and control beliefs potentially influencing AED use. The survey instrument was developed after completion of 24 iterative interviews, verified for internal validity, and piloted. Recruitment took place in June 2010 until reaching $100 \%$ of the required sample size. We measured internal consistency using Cronbach alpha and the effect of TPB constructs and specific beliefs with regression analyses. Results: Demographics of the 200 respondents: $78.8 \%$ nurses, mean age 41, $85.1 \%$ women, $52.6 \%$ college, $32.5 \%$ graduated $>20$ years ago, $66.3 \%$ full time, $33.7 \%$ allowed to use an AED, $6.7 \%$ used an AED. Median intention to use an AED during in-hospital cardiac arrest was 4.3 of 5 (5 highest) and predicted by the following TPB constructs: attitude (AdjOR 1.9; $p<.0001$ ), social influence (AdjOR 1.1; $p<.0001$ ), control beliefs (AdjOR 1.4; $p=<.0001$ ), and specific beliefs: using an
AED early could improve survival (OR $1.3 ; p<.0001)$, physicians would support my use of an AED (OR $1.1 ; p<.0001$ ), and I trust an AED to work reliably (OR $1.2 ; p<.0001)$. Conclusions: Educational interventions designed to promote the potential survival benefit of using an AED, that recruit the support of physicians, and that reassure about AED reliability are most likely to increase the use of AEDs and survival for in-hospital cardiac arrest patients. Keywords: automated external defibrillators, cardiac arrest, nursing

\section{Rowe, Brian $\mathrm{H}$}

brian.rowe@ualberta.ca

THE EFFECTIVENESS of ketorolac in the treatment of acute migraine headaches in the emergency department: a systematic review

Taggart E, Doran S, Kokotilo A, Campbell S, Villa-Roel C, Rowe BH; Department of Emergency Medicine and John Scott Library, University of Alberta, Edmonton, AB

Introduction: Acute migraine headaches are common emergency department (ED) presentations, and despite evidence for various treatments, there is conflicting evidence regarding the role of ketorolac (KET). This systematic review examined the effectiveness of parenteral KET in acute migraine. Methods: Searches of MEDLINE, EMBASE, Cochrane, CINAHL, and grey literature sources were conducted. Included studies were randomized controlled trials in which KET alone or in combination with abortive therapy was compared to placebo or other standard therapy in adult patients with acute migraine. Relevance, inclusion, and study quality were assessed independently by two reviewers, and agreement was measured using

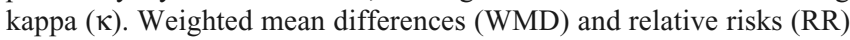
are reported with $95 \%$ confidence intervals (CIs). Results: Overall, the computerized search identified 314 citations and 1,414 grey literature citations. From a list of 10 potentially relevant studies (MEDLINE $\kappa=0.88$; EMBASE $\kappa=0.91), 8$ trials were included, involving over 321 (141 KET) patients. The median quality scores were 3 (IQR $2,4)$, and two used concealed allocation. There were no baseline differences in 10-point pain scores (WMD $=0.07$; 95\% CI $-0.39,0.54$ ). 
KET and meperidine resulted in similar pain scores at 60 minutes (WMD $=0.31 ;-0.68,1.29)$; however, KET was more effective than intranasal sumatriptan $(\mathrm{WMD}=-4.07 ; 95 \% \mathrm{CI}-6.02$ to -2.12 ). While there was no difference in pain relief at 60 minutes between KET and phenothiazine agents (WMD $=0.82 ; 95 \% \mathrm{CI}-1.33$ to 2.98 ), heterogeneity was high $(\mathrm{I} 2=70 \%)$. Side-effect profiles were similar between KET and comparison groups. Conclusions: Overall, KET is an effective second-line agent for the relief of acute migraine headache in the ED. KET is less addictive and sedating than meperidine and is more effective than sumatriptan; however, it is not as effective as metoclopramide/phenothiazine agents. Keywords: migraine, systematic review, therapy

\section{Scheuermeyer, Frank}

frankscheuermeyer@yahoo.ca

DEVELOPMENT and validation of a prediction rule for early discharge of low-risk emergency department patients with potential ischemic chest pain

Scheuermeyer F, Wong H, Yu E, Boychuk B, Innes G, Grafstein E, Gin K, Christenson J; St Paul's Hospital and the University of British Columbia, Vancouver, BC

Background: Current guidelines suggest that patients presenting to emergency departments (ED) with low-risk but potentially ischemic chest pain cannot be safely discharged without extensive investigations. We sought to derive and validate a clinical prediction rule that would miss less than $2 \%$ of patients with acute coronary syndrome (ACS) and allow $30 \%$ of patients without ACS to be discharged within 2 hours without further investigations or follow-up.

Methods: This prospective ED cohort study enrolled 1,669 consenting eligible subjects. Investigators determined 30-day outcomes according to predetermined explicit diagnostic criteria. A recursive partitioning model incorporated reliable and predictive cardiac risk factors, pain characteristics, ECG findings, and cardiac biomarker results.

Results: In the derivation cohort, 165 of 763 patients $(21.6 \%)$ had a 30 -day ACS diagnosis. The derived prediction rule was $100.0 \%$ sensitive and $18.4 \%$ specific. In the validation cohort, 119 of 906 patients (13.1\%) had ACS, and the prediction rule was $99.2 \%$ sensitive $(95 \%$ CI $95.4-100.0 \%$ ) and $23.4 \%$ specific (95\% CI 20.6-26.5\%). Based on the rule, patients have a very low risk of ACS if arrival and 2-hour troponin levels are normal, the initial ECG is nonischemic, there is no prior history of ACS or nitrate use, age is less than 50, and defined pain characteristics are met.

Conclusions: The Vancouver Chest Pain Rule defines a cohort of chest pain patients who can be safely discharged from the ED within 2 hours. Use of this rule would allow high diagnostic sensitivity while preserving health care resources. Additional validation is required. Keywords: chest pain, clinical decision rules, risk assessment

Perry, Jeffrey J

jperry@ohri.ca

COMPUTED tomography identifies patients at high risk for stroke after transient ischemic attack: a prospective, multicentre cohort study

Jason K. Wasserman, Jeffrey J. Perry, Dar Dowlatshahi, Grant Stotts, Marco L.A. Sivilotti, Andrew Worster, Marcel Émond, Jane Sutherland, Ian G. Stiell, Mukul Sharma; Department of Neurosciences, University of Ottawa, Ottawa, ON

Introduction: Ischemia on $\mathrm{CT}$ is associated with stroke following transient ischemic attack (TIA); it is unclear if specific ischemic changes predict when a patient is at highest risk for subsequent stroke. Our objective was to determine if acute ischemia, chronic ischemia, or microangiopathy, on CT, adds to the predictive power for stroke following TIA. Methods: We prospectively enrolled ED patients with
TIA and CT imaging. Primary outcome was stroke within 90 days. CT finding were based on blinded radiologist's report. Analysis used univariate and multivariate logistic regression. Results: A total of 2,028 patients were enrolled. The overall 90 -day stroke rate was $3.4 \% ; 1.5 \%$ at $\leq 2$ days and $1.9 \%>2$ days. A total of 814 patients had ischemia on CT. The increased stroke risk at 90 days was $10.6 \%$ for acute ischemia alone $(p=.002), 17.4 \%$ for acute + chronic ischemia $(p=.007), 17.6 \%$ for acute ischemia + microangiopathy $(p=.019)$, and $25.0 \%$ for acute + chronic ischemia + microangiopathy $(p=.029)$. Logistic regression analysis found acute ischemia alone, acute + chronic ischemia, acute ischemia + microangiopathy, or acute + chronic ischemia + microangiopathy had $3-(\mathrm{OR}=2.61 ; 95 \% \mathrm{CI} 1.22-5.57 ; p=.013), 5-(\mathrm{OR}=$ $5.35 ; 95 \%$ CI $1.71-16.70 ; p=.004), 5-(\mathrm{OR}=4.90 ; 95 \%$ CI $1.33-$ $18.07 ; p=.017)$, and 8 -fold (OR $=8.04 ; 95 \%$ CI $1.52-42.63 ; p=.014)$ greater risk of stroke, respectively. In the subgroup with stroke $>2$ days, only acute ischemia (OR $=2.70 ; 95 \%$ CI $1.01-7.18 ; p=.047)$ and acute \pm chronic ischemia (OR $=1.98 ; 95 \%$ CI 1.04-3.78; $p=.039$ ) had increased stroke risk. Conclusion: In ED TIA patients, acute ischemia alone or acute ischemia with chronic ischemia and/or microangiopathy was associated with high 90-day risk of stroke. Patients with acute or chronic ischemia are at highest risk for stroke after 2 days. Keywords: computed tomography, prognosis, transient ischemic attack

\section{Stiell, Ian G}

iharris@ohri.ca

A PREDICTION rule for emergency department patients with chronic obstructive pulmonary disease

Ian G. Stiell, Catherine M. Clement, Brian H. Rowe, Robert J. Brison, Eddy Lang, Bjug Borgundvaag, Shawn Aaron, Lisa A. Calder, Jeffrey J. Perry, Alan Forster, George A. Wells; Department of Emergency Medicine, University of Ottawa, Ottawa, ON

Introduction: ED physicians frequently treat and make disposition decisions for patients with acute exacerbations of COPD. Our goal was to derive a prediction rule to stratify the risk of poor outcomes for COPD patients. Methods: This multicentre, prospective cohort study was conducted in six academic EDs and included adults who presented with an exacerbation of COPD. We assessed patients for 79 standardized clinical and laboratory variables, including a structured 3-minute walk test, conducted after treatment. We followed both admitted and discharged patients for serious adverse events (SAEs), which were defined as death, intubation, admission to a monitored unit, or relapse back to the ED requiring admission within 14 days. We conducted stepwise logistic regression analyses to calculate adjusted odds ratios for predictors of SAE and then developed a risk stratification prediction rule. Results: The characteristics of the 945 study patients were mean age 72.6 , male $51.6 \%$, EMS arrival $48.3 \%$, admitted to hospital $37.5 \%$, mortality $1.0 \%$. There were 74 (7.8\%) SAE cases, and 36 occurred in patients discharged on the initial ED visit. We developed a multivariate model consisting of 10 clinical and laboratory predictors with Hosmer-Lemeshow goodnessof-fit $p$ value .703 and area under ROC curve of 0.796 (95\% CI 0.74 $0.85)$. Rounding coefficients led to a prediction rule with scores 0 to 16 , representing risk strata of SAE from 2.2 to $91.4 \%$. Internal validation using the bootstrap method showed the risk scores to be very accurate across 1,000 replications. Choosing a score of 1 as the threshold for admission would be $91.2 \%$ sensitive for SAE and would require that only $57.6 \%$ of patients be admitted. Conclusions: This COPD prediction rule should help ED physicians stratify the risk of poor outcomes for patients with COPD exacerbation and lead to safe, evidence-based disposition decisions. This will improve patient safety and rationalize admission decisions. Keywords: chronic obstructive pulmonary disease, decision support techniques, risk assessment 
Stiell, Ian G

iharris@ohri.ca

IMPLEMENTATION of a c-spine clearance protocol by emergency department nurses

Ian G. Stiell, Catherine M. Clement, Jamie Brehaut, Jeremy Grimshaw, Annette O'Connor, Jeffrey J. Perry, George A. Wells, Taryn MacKenzie, Christine Beland, Pamela Sheehan, Barbara Davies; Department of Emergency Medicine, University of Ottawa, Ottawa, ON

Introduction: Prolonged immobilization of minor trauma patients adds to ED congestion and is very uncomfortable for patients. Our goal was to evaluate the impact and safety of implementing a medical directive allowing nurses to remove the c-spine immobilization of trauma patients judged to be low risk by a specific c-spine clearance protocol (CCP) that had been previously validated. Methods: We conducted a prospective cohort study in two large university hospital EDs and enrolled alert and stable adult trauma patients who presented with neck pain or were immobilized on an EMS backboard. ED triage nurses had been trained on the CCP by a CD and hands-on sessions, had to have accurately evaluated 10 patients and three interobserver cases, and had passed a written test prior to being certified to clear the c-spine. We evaluated safety and impact from study data forms, imaging records, and 30-day follow-up using descriptive statistics with $95 \%$ CIs Results: We enrolled 1,608 patients over 24 months: mean age 42.3 years, motor vehicle collision $59.6 \%$, ambulance arrival $81.3 \%$, admitted $3.9 \%$, c-spine fracture $1.5 \%$. Overall, 83 nurses participated and removed immobilization from $33.0 \%$ (95\% CI 30.7-35.3\%) of patients and were able to have $24.0 \%$ (95\% CI $21.9-26.1 \%$ ) of all cases walk to the ambulatory area of the ED. Nurses were 100\% sensitive for identifying patients with c-spine injuries and showed $99.6 \%$ agreement with the criterion interpretation of the CCP by the investigators. No patients suffered adverse outcomes or neurologic deficit. In only $4.3 \%$ of cases did nurses report uneasiness with application of the CCP. Conclusions: We have shown the effectiveness and safety of training and empowering ED triage nurses to evaluate and clear the c-spine of alert, stable trauma patients. This strategy should improve patient flow in our crowded EDs, diminish unnecessary patient discomfort, and increase ED nurse decision making. Future initiatives should address knowledge translation to multiple sites. Keywords: cervical spine injury, decision support techniques, nursing

Stenstrom, Rob

robstenstrom@shaw.ca

PREVALENCE of HIV in a Canadian inner-city emergency department

Stenstrom R, Grafstein E, Poureslami I, Sherlock C, Harris D, Scheuermeyer F, Hunte G, Gustafson R, Barrios R, Montaner J; University of British Columbia Department of Emergency Medicine and St Paul's Hospital, Vancouver, BC

Objective: (1) To estimate the seroprevalence of HIV infection in an inner-city Canadian emergency department (ED); (2) to measure patient satisfaction with point-of-care (POC) HIV antibody testing in the ED. Methods: This was a prospective, IRB-approved cohort study performed in an urban tertiary care ED between August 2009 and November 2010. We hypothesized that 2 to $4 \%$ of our patient population would be HIV positive. Based on a sample size calculation for a $95 \%$ confidence interval $(\mathrm{CI}) \pm 1 \%$, around a prevalence of $3 \%, 1,216$ subjects were required. Consecutive patients in the ED were enrolled using probabilistic sampling of times of day/night and days of the week, based on patient volumes. Inclusion criteria: age 19 to 75 years of age and understand/provide informed consent. Exclusion criteria: intoxicated or in extremis. Following informed consent, subjects HIV antibody status was obtained using the Biolytical INSTI POC test. Subjects completed a questionnaire regarding HIV risk factors, knowl- edge, and perceived HIV status. Subjects with a positive test received confirmatory Western blot testing. HIV-positive subjects were offered immediate referral to an HIV specialty clinic and counseling if they were not aware of their HIV status. Results: A total of 2,001 patients were approached for the study, and 1,402 agreed to participate (70.1\%). Participants were similar to nonparticipants for age, gender, and CTAS level. Mean age of subjects was 43.3 years; $58.4 \%$ of subjects were male. HIV seroprevalence was 65 of $1,402(4.6 \%$; $95 \% \mathrm{CI}$ $3.5-5.8 \%$ ). No previously undiagnosed HIV+ patients were identified in this cohort. Patient satisfaction with POC HIV testing was high (mean satisfaction score 9.6 of 10 ; $\mathrm{SD}=0.91$ ). Conclusions: Based on a rapid, POC HIV antibody test, the seroprevalence rate of HIV in an inner-city ED was slightly higher than expected at $4.6 \%$. POC testing in the ED is feasible, and patients' satisfaction with this procedure was high. Keywords: human immunodeficiency virus, point-of-care systems, prevalence

Innes, Grant

grant.innes@albertahealthservices.ca

REGIONAL implementation of new intake processes improves access to care for midlevel acuity patients in three urban emergency departments

Grant Innes, Lester Mercuur, Dongmei Wang, Shawn Dowling, Eddy Lang; University of Calgary, Calgary, AB

Introduction: The evaluation of midlevel acuity patients who present to the ED is often hampered by access block. The goal of this study was to describe the impact of a three-phase evolution in re-engineered processes of care for midlevel acuity patients instituted in a three-hospital region serving over 1 million inhabitants. Methods: Using administrative databases, access and quality of care were compared during three 5- to 10-month periods from 2008 to 2010. Phase I (2008) served as a control and involved MDs waiting for patients to arrive in traditional care spaces. In phase II (2009), known as waiting room care (WRC), MDs assessed patients in spaces located off the main ED. In phase III (2010), known as triage-in (TI), patients were evaluated in dedicated spaces linked to a formal process of care. In both of these latter phases, patients requiring additional therapy or observation could be transferred to treatment or "awaiting results" zones. Primary outcomes were median time from triage to MD assessment, percentage of patients seen in WRC/TI, left without being seen (LWBS), and unplanned revisits (UR). Kruskal-Wallis and chi-square were used for comparisons. All CTAS III patients were included in the analysis. Results: A total of 62,917 CTAS III visits were included. The proportion treated in TI/WRC areas increased from 4.3 to 26.1 to $51.7 \%$ ( $p<$ .001 ) over the three phases. Median triage to MD assessment time fell significantly over the three phases of the study (157 versus 149 versus 135 minutes, respectively; $p<.001$ for all comparisons). There was a reduction in LWBS from $12.5 \%$ to $10.9 \%$ to $8.6 \%$ over the three periods; $p<.001$ ). UR within 72 hours was unaffected over the latter two phases; $7.1 \%$ v. $8.9 \%$ v. $8.8 \%$, respectively ( $p=$ NS for latter two). Potential confounders did not influence these results. Conclusion: We describe the impact of the first regionally implemented program for improving ED access times yielding significant improvements in access to care and generated improvements in quality of care. Keywords: administrative database, emergency services, health services accessibility, hospital/accessibility

\section{Gouin, Serge}

sergegouin@aol.com

A RANDOMIZED double-blind trial comparing the effect of oral dimenhydrinate versus placebo in children with moderate vomiting from acute gastroenteritis

Gouin S, Vo TT, Roy M, Lebel D, Gravel J; CHU Ste-Justine, Emergency Medicine, Montréal, QC 
Introduction: No clinical trial has evaluated the efficacy of dimenhydrinate if given orally in children with acute gastroenteritis. Objective: To evaluate the efficacy and safety of oral dimenhydrinate in children with vomiting due to gastroenteritis. Methods: This was a randomized, double-blinded, controlled trial conducted in an academic pediatric emergency department. Children 2 to 12 years old who presented with at least five episodes of vomiting in the previous 12 hours and diagnosed with gastroenteritis were randomized to dimenhydrinate PO (1 $\mathrm{mg} / \mathrm{kg}, \max =50 \mathrm{mg}$ ) every 6 hours for 4 doses or placebo for 4 doses. The primary outcome measure was the number of good outcome, defined as one episode or less of vomiting 24 hours after the first dose of drug administration. An intention-to-treat analysis was performed Results: A total of 209 patients were eligible; 50 refused to participate, and 7 were missed. Of the 152 recruited patients, 8 were lost to followup. There were no statistically significant differences in demographics and clinical characteristics at baseline between the two groups. The proportions of participants showing failure of treatment as defined by more than one vomiting episode in the 24 hours following recruitment were similar for both treatment groups: diphenhydramine 0.31 vs placebo 0.29 (difference 0.02 : $95 \%$ CI -0.12 to 0.17 ). The Pearson chisquare test showed no statistical association between treatment and occurrence of more than one vomiting episode in the 24 hours following randomization (chi-square 584; 1 DOF; $p$.445). There were no statistical differences between the two groups for rates of IV insertion, mean total number of episodes of vomiting and diarrhea, abdominal pain, nausea, duration of symptoms, revisit rates, and parental absenteeism. The proportions of reported side effects were high but similar in both groups $(53 \%$ v. $54 \%)$. Conclusions: The prescription of oral dimenhydrinate does not appear to decrease the frequency of vomiting in children with vomiting from an acute gastroenteritis compared to a placebo. Keywords: gastroenteritis, randomized controlled trial, vomiting/drug therapy

Aguanno, Alaina F

aaguanno@ucalgary.ca

DRIVERS of patient experience: findings from the Health Quality Council of Alberta Urban and Regional Emergency Department Patient Experience Report

Cooke T, Aguanno A, Schorn R, Lahtinen M, Cowell J, MacRae A, Rowe B, Lang E; Health Quality Council of Alberta, Calgary, AB

Introduction: This study examines the magnitude of influence of specific modifiable factors (ie, wait time) on patient ED experience. Methods: Cross-sectional survey of a random patient sample from 12 urban/regional EDs in winter 2007 and 2009. A previously validated questionnaire, based on the British Healthcare Commission Survey, was distributed according to a modified Dillman protocol. Exclusion criteria: age 0 to 15 years, left prior to being seen/treated, died during the ED visit, no contact information, presented with a "privacy"-sensitive case. Nine previously identified and validated composite variables (staff care/communication, respect, pain management, wait time/crowding, facility cleanliness, discharge communication, wait time communication, medication communication, and privacy) were evaluated for their influence on patients' overall rating of care. Composite variables and rating of care were rated on a scale from from 0 (lowest) to 100 (highest). Calculated influence is represented as a standardized composite coefficient. Results: A total of 21,639 surveys were distributed. Response rate was $46 \%$. Composite variable coefficients were staff care/communication (0.38), respect (0.17), pain management (constituent variables not comparable so decomposed for regression), wait time/crowding (0.09), facility cleanliness (0.13), discharge communication $(0.10)$, wait time communication (0.03), medication communication (no significant influence), and privacy $(0.02)$ Thus, if a patient's staff care/communication composite score increases from 50 of 100 to 70 of 100, an initial global rating of care of 70 of
100 is predicted to increase to 78 of 100 . Path analysis showed cascading effects of pain management and wait time on other variables. Variable coefficients affect patients according to situational relevance. Conclusions: Global patient ED experience is most strongly influenced by staff care/communication. Pain management and wait time synergistically effect downstream variables. Efforts to improve patient satisfaction should focus on these factors. Keywords: emergency service/hospital, patient satisfaction, survey

Stiell, Ian G

iharris@ohri.ca

CANADIAN Heart Failure Risk Scale to identify emergency department patients with heart failure at high risk for serious adverse events

Ian G. Stiell, Catherine M. Clement, Robert J. Brison, Brian H. Rowe, Bjug Borgundvaag, Shawn Aaron, Eddy Lang, Lisa A. Calder, Jeffrey J. Perry, Alan Forster, George A. Wells; Department of Emergency Medicine, University of Ottawa, Ottawa, ON

Introduction: There are no validated decision tools to assist physicians with difficult hospital admission decisions for ED patients with heart failure (HF). We sought to develop a risk scoring system to identify HF patients at high risk for serious adverse events (SAE). Methods: We conducted a prospective cohort study in six large EDs and enrolled adult patients who presented with dyspnea secondary to HF. Each patient was assessed for standardized clinical and laboratory variables, including the novel 3-minute walk test and quantitative NTProBNP. Patients, both those admitted and discharged, were followed for SAE, which was defined as death, intubation, admission to a monitored unit, or relapse back to the ED requiring admission within 14 days. We calculated adjusted odds ratios for predictors of SAE by stepwise logistic regression and then developed a scoring system by rounding coefficients. Results: We enrolled 559 patients with mean age 76.0 years and overall hospital admission of $38.1 \%$. Of $65(11.6 \%)$ SAE cases, $31(47.7 \%)$ occurred in patients not admitted on the initial ED visit. The multivariate model and resultant Canadian Heart Failure Risk Scale consist of 10 elements, and SAE risk varied from $2.8 \%$ for a score of 0 to $89.0 \%$ for a score of 9 , with good calibration between observed and expected probabilities. Internal validation showed the risk scores to be very accurate across 1,000 replications using the bootstrap method. Choosing a threshold of 1,2 , or 3 total scores for admission would be associated with sensitivities of $95.2 \%, 80.6 \%$, or $64.5 \%$, respectively, all better than current practice. Conclusions: While some HF patients seen in the ED suffer subsequent SAEs or death, the majority have good outcomes even if initially discharged. We have developed an accurate and easy to use Canadian Heart Failure Risk Scale that can be used to stratify the risk of poor outcomes for these patients and to enable rational and safe hospital admission decisions. Keywords: congestive heart failure, prognosis, risk assessment

Austin, Michael A

maustin@toh.on.ca

*A RANDOMIZED clinical trial of continuous positive airway pressure (CPAP) in the treatment of acute cardiogenic pulmonary edema (ACPE) patients in the prehospital setting

Michael A. Austin ${ }^{1,2,3}$, Karen E. Wills ${ }^{1}$

${ }^{1}$ Menzies Research Institute Tasmania, Hobart, TAS, Australia; ${ }^{2}$ Tasmanian Ambulance Service, Hobart, TAS, Australia; ${ }^{3}$ Department of Emergency Medicine, University of Ottawa, Ottawa, ON

Introduction: The prehospital use of continuous positive airway pressure (CPAP) ventilation is a relatively new management for acute cardiogenic pulmonary edema (ACPE), and there is little high-quality evidence on the benefits or potential dangers in this setting. The aim of this study was to determine whether patients in severe respiratory dis- 
tress treated with CPAP in the prehospital setting have a lower mortality than those treated with usual care. Methods: Randomized, controlled trial comparing usual care versus CPAP (Whisperflow) in a prehospital setting, for adults experiencing severe respiratory distress, with falling respiratory efforts, due to a presumed ACPE. Patients were randomized to receive either usual care, including conventional medications (nitrates, furosemide, and oxygen) plus bag-valve-mask ventilation, versus conventional medications plus CPAP. The primary outcome was prehospital or in-hospital mortality. Secondary outcomes were need for tracheal intubation, length of hospital stay, change in vital signs, and arterial blood gas results. We calculated relative risk with $95 \%$ CIs. Results: Fifty patients were enrolled with mean age 79.8 (SD 11.9), male $56.0 \%$, mortality $20.0 \%$. The risk of death was significantly reduced in the CPAP arm with mortality $34.6 \%$ ( 9 deaths) in the usual care arm compared to $4.2 \%$ (1 death) in the CPAP arm (RR, $0.12 ; 95 \%$ CI 0.02 to $0.88 ; p=.04$ ). Patients who received CPAP were significantly less likely to have respiratory acidosis (mean difference in $\mathrm{pH} 0.09 ; 95 \% \mathrm{CI} 0.01$ to $0.16 ; p=.02 ; n=24$ ) than patients receiving usual care. The length of hospital stay was significantly less in the patients who received CPAP (mean difference 2.3 days; $95 \% \mathrm{CI}$ -0.01 to $4.6, p=.05$ ). Conclusions: We found that CPAP significantly reduced mortality, respiratory acidosis, and length of hospital stay for patients in severe respiratory distress caused by ACPE. This study shows the use of CPAP for ACPE improves patient outcomes in the prehospital setting. Trial reg. ANZCTR ACTRN12609000410257

Keywords: acute cardiogenic pulmonary edema, continuous positive airway pressure, prehospital care

Stiell, Ian G

iharris@ohri.ca

ASSESSING cognition in the emergency department: prospective validation of the Ottawa 3DY case finding tool with Animal Fluency Test

Laura J.Y. Wilding, Ian G. Stiell, Frank Molnar, Jo-Anne O’Brien, Joy Moors, William B. Dalziel; School of Nursing, University of Ottawa, Ottawa, ON

Introduction: Older adults frequently present to the $\mathrm{ED}$ with cognitive impairment, but assessment is challenging as few tools exist that can be quickly administered by front-line practitioners in the ED. This study validated use of the Ottawa 3DY (O3DY), a recently derived four-question tool designed to screen for cognitive impairment in the elderly, and compared its performance to the Animal Fluency Test (AFT). Methods: A prospective cohort study was conducted in two EDs of a large multicampus academic health sciences centre and enrolled a convenience sample of medically stable patients aged 75 years and older with no history of cognitive impairment. Eligible patients were assessed by experienced geriatric emergency management (GEM) nurses who administered the Mini Mental State Exam (MMSE) ordered with the O3DY questions first, followed by the AFT. Patients meeting criteria for the GEM program also received a comprehensive geriatric evaluation, including a cognitive assessment and referrals to specialized geriatric services as indicated. We calculated sensitivity and specificity of the O3DY and AFT versus the MMSE. Results: The 238 study patients (mean age 81.9 , female $60.1 \%$, admitted $26.5 \%$ ) had these proportions for standard score cutoffs: MMSE $<$ $25,13.5 \%$; AFT $<10,27.3 \%$; and O3DY $<4,36.1 \%$. The O3DY and MMSE were in agreement for $75.6 \%$ of cases, with O3DY identifying 30 of 32 cases with MMSE < 25 (sensitivity 93.8\% [95\% CI 85.4$100 \%$ ]; specificity $23.5 \%$ [95\% CI $21.1-33.3 \%]$ ]). The AFT and MMSE were in agreement for $76.1 \%$ of cases, with AFT identifying only 20 of 32 cases with MMSE < 25 (sensitivity $62.5 \%$ [95\% CI 45.7-79.3\%]; specificity 78.2\% [95\% CI 72.5-83.8\%]). Conclusions: The O3DY, but not the AFT, showed excellent sensitivity versus the MMSE. The O3DY is an effective tool that could be used by ED nurses and physicians to screen for cognitive impairment in older adults, thus identifying those who need further assessment and appropriate management. Keywords: cognitive impairment, prospective studies, screening tools

Gravel, Jocelyn

graveljocelyn@hotmail.com

VALIDITY of the Canadian Triage and Acuity Scale for children: a prospective multicentre study

Jocelyn Gravel, Serge Gouin, Ran D. Goldman, Martin H. Osmond, Eleanor Fitzpatrick, Kathy Boutis, Chantal Guimont, Gary Joubert, Kelly Millar, Sarah Curtis, Douglas Sinclair, Devendra Amre; Département de pédiatrie, CHU Sainte-Justine, Montréal, QC

Background: The Canadian Triage and Acuity Scale (CTAS) is a fivelevel triage tool constructed from a consensus of experts and is universally used in Canada. Objective: The aim of the study was to evaluate the validity of the CTAS as measured by the correlation between triage level and proxy markers of severity. Methods: This was a prospective cohort study evaluating a sample of children presenting to emergency departments (EDs) who were initially triaged from level 2 (emergent) to 5 (nonurgent) on the CTAS. It was performed in nine tertiary care pediatric EDs across Canada. Research shifts of 8 hours distributed among days, evenings, and nights were performed once a month for 12 months in each setting. A dedicated research nurse recruited patients just after their triage. The primary outcome was the correlation between triage level and hospitalization. Secondary outcomes included resource use (laboratory testing, intravenous hydration or medication, or surgical procedure) and length of stay in the ED. The correlation between triage level and categorical outcomes was evaluated by a chisquare test. ANOVA was performed to evaluate length of stay in the ED among the different triage levels. Based on a pilot study, it was estimated that the recruitment of 1,000 patients would provide at least 50 participants per triage category and narrow confidence intervals for the outcome proportions. Results: A total of 1,313 patients were recruited and included in the final analysis. Hospitalization proportion was of $36 \%$ for patients triaged at level $2,9.0 \%$ at level $3,2.4 \%$ at level 4 , and no admittance for patients triaged at level 5 (chi-square 130.762, DOF $3 ; p<.001$ ). There was also a strong association between triage levels and use of any medical resources (chi-square 69.517 , DOF $3 ; p<.001)$ and length of stay $(p<.001)$. Conclusion: The CTAS is a valid triage tool as demonstrated by its good correlation with hospitalization, resource use, and length of stay in the ED. Keywords: Canadian Triage and Acuity Scale, pediatrics, validity

Clement, Catherine M.

cclement@ohri.ca

PERCEIVED facilitators and barriers to implementation of a cspine clearance protocol by emergency department nurses

Catherine M. Clement, Ian G. Stiell, Taryn MacKenzie, Christine Beland, Pamela Sheehan, Jamie Brehaut, Connor Sheehan, Annette O'Connor, and Barbara Davies; Department of Emergency Medicine, Ottawa Hospital Research Institute, Ottawa, ON

Introduction: We conducted an implementation study on use of a validated c-spine clearance protocol (CCP) by emergency department (ED) nurses, using a medical directive, to clear the c-spine of minor trauma patients $(N=1,608)$. This survey sought to identify potential facilitators and barriers to implementation. Methods: We conducted a handdelivered paper survey of ED triage nurses who participated in the CCP project and had removed c-spine immobilization of trauma patients judged to be low risk, via medical directive, at two large university hospital EDs. The medical directive had been in place for 18 months. Twenty-five questions on potential facilitators and barriers were asked using a 6-point Likert scale including practice patterns, 
aids, and problems identified in application. Two open-ended questions allowed further opportunity to record additional facilitators and barriers. A modified Dillman technique was used involving several distributions of the survey. We calculated descriptive statistics with $95 \%$ CIs. Results: The 55 respondents, representing a $77.5 \%$ response rate, had these characteristics: female $71.1 \%$, mean years in nursing 20.9 , and mean years in ED 13.1. Of the nurses, 91.2\% (95\% CI 83-99\%) indicated that they were comfortable applying the CCP and $98.2 \%$ (95\% CI 94-100\%) responded that the CCP was useful to their practice. Other responses included nurse role models in unit important, $100 \%$; CCP increases autonomy, $98.2 \%$; efficient use of time, $98.2 \%$; increased chance of lawsuits, $18.2 \%$; not safe for patients, $7.3 \%$. Examples of potential facilitators included "good peer support" and "reduction in ambulance offload times/clearing busy ED." Barriers included "perceived lack of time" and "vague history." Conclusions: The majority of ED triage nurses were comfortable following a medical directive that allows removal of c-spine immobilization on minor trauma patients. Most nurses embraced the increased autonomy and decision making in this strategy that speeds patient care in crowded EDs. Keywords: Canadian c-spine rule, implementation, nursing

\section{Chan, Teresa M.}

teresa.chan@medportal.ca

*UNDERSTANDING communication between emergency and consulting physicians: a qualitative study that defines the essential elements of a referral-consultation

Teresa Chan, BEd, MD; Donika Orlich, BSc; Kulamakan Kulasegaram, HBSc; Jonathan Sherbino, MD, MEd; Division of Emergency Medicine, McMaster University, Hamilton, ON

Introduction: Effectively communicating with consultants is a key skill for emergency physicians. Our objective was to define the essential elements of an effective referral-consultation between emergency and consulting physicians. Methods: From March to September 2010, 61 physicians (31 residents and 30 attendings; 21 EM, 20 GS, 20 IM) were interviewed by a single interviewer. Two investigators independently reviewed $100 \%$ of the transcripts using grounded theory to generate a code of categories until saturation was reached. Coding disagreements were resolved by consensus, yielding a single inventory of themes, subthemes, and qualifiers. All of the transcripts were coded using the common code; $30 \%$ of the transcripts were coded by a second investigator to determine interrater agreement. Results: A total of 245 themes and qualifiers were identified. Interrater agreement was $77 \%$. Shared themes (> $60 \%$ endorsment) in the referral-consultation process were initial preparation (overall endorsement $87 \%$ [ranging from $70-100 \%$ in different groups]), basic communication (ie, identify physician by name) $(100 \%)$, hypothesis of patient's presentation $(75 \%$ [62-83\%]), clinical question for consultant (70\% [55-95\%]), urgency (100\%) and patient stability (74\% [62-80\%]), clarifiying questions (100\%), follow-up communication (98\% [95-100]\%), and feedback (98\% [95-100\%]). Each clinical specialty significantly contributed to the model $\left(\chi^{2}=7.879 ; p\right.$ value $=.019)$; however, each of the groups contributed different amounts (EM 57\%, GS 41\%, IM 64\%). Conclusions: We define the essential elements of a referral-consultation with input from emergency and consultant physicians. These findings may inform the development of referral-consultation model for junior learners. Keywords: communication skills, qualitative research, specialist consultation

Frank, Jason

jfrank@rcpsc.edu

EMERGENCY department flow: impact of a brief educational intervention on physician productivity

Jason R. Frank, A. Curtis Lee, Mathieu Gatien, Eric Clark, A. Adam Cwinn; Department of Emergency Medicine, University of Ottawa, Ottawa, ON
Introduction: Little is known about how to improve the productivity of individual emergency physicians, in terms of patients seen per hour, despite its importance for ED patient flow and overcrowding. We studied the impact of a brief educational intervention on emergency physician throughput in a single academic centre. Methods: We conducted an educational cohort study involving 42 emergency physicians divided into intervention (IG) $(n=26)$ and control (CG) $(n=16)$ groups. IG members participated in a case-based educational session on principles of ED flow effectiveness. A physician productivity index consisting of the number of patients seen in a month weighted to shift type and time of day was compared between groups and with historical controls. An index value of 0.00 represented a physician seeing the expected number of patients per hour during their monthly shifts. A positive value represented seeing more than the expected number of patients per hour. The primary outcome measure was change in the group mean index before and after the intervention. Satisfaction with the educational intervention was assessed. Results: There was no significant difference in the productivity index between the IG and CG at baseline in the same time period 1 year earlier. After the intervention, IG physicians' mean productivity increased versus historical controls $(-0.08$ in 2009 and +0.04 in 2010, $t=2.3, p<.05)$, while CG productivity did not. Productivity index scores between the IG and CG did not achieve statistical significance (means $=+0.04$ and -0.10 , respectively, $t=1.29, p>.05$ ). Cohen's $\mathrm{d}$ for the intervention was 0.4 , suggesting a moderate educational effect. IG participants rated the intervention highly. Conclusions: This is the first ED productivity education program of its kind to show an impact on patients seen per hour by academic faculty physicians. Further studies are needed to validate and refine the methods to improve physician productivity in the ED setting. Keywords: educational intervention, emergency department flow, physician productivity

\section{Gravel, Jocelyn}

graveljocelyn@hotmail.com

A PROSPECTIVE multicentre evaluation of the reliability of the Canadian Triage and Acuity Scale for children

Jocelyn Gravel, Serge Gouin, Ran D. Goldman, Martin H. Osmond, Eleanor Fitzpatrick, Kathy Boutis, Chantal Guimont, Gary Joubert, Kelly Millar, Sarah Curtis, Douglas Sinclair, Devendra Amre; Département de Pédiatrie, CHU Sainte-Justine, Montréal, QC

Background: The Canadian Triage and Acuity Scale (CTAS) is a five-level triage tool constructed from a consensus of experts and is universally used in Canada. Objective: The aim of the study was to measure the interrater agreement of nurses assigning triage levels using the CTAS to children (0-18 years old) visiting multiple pediatric emergency departments (EDs) using the CTAS. Methods: This was a prospective cohort study evaluating children presenting to pediatric EDs who were initially triaged from level 2 (emergent) to 5 (nonurgent) on the CTAS. It was performed in nine tertiary care pediatric EDs across Canada in 2009-2010. In each setting, a research nurse performed one recruitment shift per month for 12 months (5 days, 5 evenings, and 2 nights). She recruited eligible patients just after their initial triage to perform a second triage. Both triages were performed blinded to the other. The primary outcome measure was the interrater agreement between the two nurses measured by the weighted kappa score. Based on a pilot study, we estimated that the recruitment of 1,500 patients would provide a $95 \%$ confidence interval for linear weighted kappa of \pm 0.05 . Results: A total of 1,411 patients were approached, and 1,313 agreed to participate. The main reasons for refusal were refusal of a second exam $(n=52)$, immediate care $(n=26)$, and absence of a parent for informed consent $(n=21)$. All participants were included in the analysis except one patient with missing triage level data. The overall interrater agreement was good as demonstrated by the linear weighted kappa score of $0.60(95 \% \mathrm{CI}$ 
$0.57-0.64)$ and quadratic weighted kappa score of 0.67 (95\% CI 0.55 0.78). There was a discrepancy of more than one level in only two patients. Conclusion: The CTAS demonstrates good interrater agreement between nurses assigning triage level to children presenting to multiple pediatric EDs. Keywords: Canadian Triage and Acuity Scale, children, reliability

Lang, Eddy S

eddy.lang@albertahealthservices.ca

ADMISSION rates for older emergency department patients with syncope are higher in the United States than Canada

Eddy Lang, Ruwan Ekanayake, Brian Rowe, Venkatesh Thiruganasambandamoorthy, Robert Sheldon, Barnet Eskin, John R. Allegra University of Calgary, Calgary, AB

Introduction: Syncope is a common emergency department (ED) presenting complaint; admission rates for syncope vary widely, and hospitalization is often of little benefit. No previous study has compared US admission rates for syncope with those in other countries. Moreover, there are no studies reporting data for higher-risk, older patients who are frequently admitted. Our objective was to compare admission rates for patients $>60$ years of age presenting to US and Canadian EDs with syncope. Methods: Design: Retrospective cohort of ED visits. Setting. Twenty New York and New Jersey EDs with annual visits from 22,000 to 82,000 and 104 EDs in Alberta with 5,000 to 70,000 average annual visits matched for age range and year of presentation. Population: Consecutive patients seen by ED physicians in the year 2009. Protocol: We identified syncope visits using ICD codes for patients $>60$ years old and calculated the proportion hospitalized. We included all visits where syncope was either the primary or secondary diagnosis. We used Student $t$-test $($ alpha $=0.05)$ to compare differences and calculated $95 \%$ confidence intervals (CI). Results: There were 978,977 and 1,972,924 total ED visits in the US and Canadian EDs, respectively. Of these, 7,676 and 4,458 were for syncope in patients $>60$ years old, respectively. The mean age was 78 and 79 years, and $56 \%$ and $52 \%$ were female, respectively. The proportion hospitalized was $72.4 \%$ in the United States and $27.1 \%$ in Canada (difference $=45.3 \%$, 95\% CI 43.7 to $46.9 \%[p<.001])$. Confining the analysis to patients $>80$ years old yielded similar results. Conclusions: We found that admission rates for syncope in patients $>60$ years of age in the United States were higher than in Canada. We speculate that this may be due to lower physician risk tolerance in the United States and also to lack of clinical management consensus and valid decision aids for serious adverse events. Keywords: admission rates, retrospective cohort, syncope

Aguanno, Alaina F

aaguanno@ucalgary.ca

PERCEPTIONS of urgency: findings from the Health Quality Council of Alberta Urban and Regional Emergency Department Patient Experience Report

Aguanno A, Cooke T, Cowell J, Rowe B, MacRae A, Lang E; University of Calgary, Calgary, $\mathrm{AB}$

Introduction: Emergency department (ED) patients are assigned a score according to the Canadian Emergency Department Triage and Acuity Scale (CTAS). An understanding of patient self-triage abilities will inform health policies targeting how/where patients access the health care system. Methods: Cross-sectional survey of a random patient sample from 12 urban/regional EDs in winter 2007 and 2009. A previously validated questionnaire, based on the British Healthcare Commission Survey, was distributed according to a modified Dillman protocol. Exclusion criteria: age $0-15$ years, left prior to being seen/treated, died during ED visit, no contact information, presented with a "privacy"-sensitive case. Alberta Health Services provided linked nonsurvey data. Results: A total of 21,639 surveys were distrib- uted. Response rate was $46 \%$. Patients rated health problems as "lifethreatening" (6\%), "possibly life-threatening" $(22 \%)$, "urgent" $(30 \%)$, "somewhat urgent" (37\%), or "not urgent" $(5 \%)$. Triage nurses assigned the same patients CTAS scores of I $(<1 \%)$, II $(20 \%)$, III (45\%), IV (29\%), or V (5\%). Patients self-rated their condition as 3 or 4 points less urgent than the assigned CTAS score $(<1 \%$, respectively), 2 points less urgent (5\%), 1 point less urgent (25\%), exactly as urgent $(38 \%), 1$ point more urgent $(24 \%), 2$ points more urgent $(7 \%)$, or 3 or 4 points more urgent ( $1 \%$, respectively). Among CTAS I or II patients, 54\% described their problem as "(possibly) life-threatening," $26 \%$ as "urgent: risk of permanent damage," $18 \%$ as "urgent: needed to be seen that day," and $2 \%$ as "not urgent: wanted to be seen that day." The latter two groups represent $4 \%$ of the sample population. Conclusions: Most ED patients are aware of their acuity. Encouraging patients with low-urgency conditions to self-triage to lower-acuity sources of care may relieve stress on EDs. However, physicians and patients must be aware that $\approx 4 \%$ of patients are unable to self-triage safely. Keywords: Canadian Triage and Acuity Scale, patient perception, survey

Stiell, Ian G

iharris@ohri.ca

DEVELOPMENT of a symptom-specific quality of life scale in emergency department patients with recent-onset atrial fibrillation/flutter

Debra Eagles, Jeff H. Yoo, Catherine M. Clement, Ian G. Stiell; Department of Emergency Medicine, University of Ottawa, Ottawa, ON

Introduction: Recent-onset atrial fibrillation/flutter (RAFF) is the most common arrhythmia requiring management in the ED. The purpose of this study was to develop a symptom-specific quality of life (QoL) measure as both a clinical and a research tool for patients presenting to the ED with RAFF. Methods: We conducted a prospective cohort study in the EDs of two university hospitals and enrolled adult patients presenting with palpitations. We adapted the ED RAFF Scale from the Canadian Cardiovascular Society Severity of AF Scale and created seven symptom questions. Patients were asked to rate the severity of each symptom on a scale from 0 to 10 , with 0 being "none" and 10 being "worst possible." Patients were randomized to self- or clinician administration of the ED RAFF Scale and the validated, generic SF-8 QoL Scale at time 0 before crossing over at time 15 . We compared results with Student $t$-test. Results: We enrolled 68 patients: male $58.8 \%$, mean age 61.2 years, mean duration symptoms 11.7 hours. Individual items on the ED RAFF Scale had these means (SD): palpitations 2.8 (2.7), lightheadedness 2.0 (2.4), chest pain 1.8 (2.3), shortness of breath $2.2(2.7)$, shortness of breath with exertion 6.3 (2.8), fatigue 3.9 (3.1), fatigue with exertions 6.7 (2.8). There was no difference between scores for self- v. clinician completion (all $p$ values $>$.40). High values (7-10) for all ED RAFF Scale questions showed good discrimination for both the physical and mental composite scores of the SF-8 (all $p$ values <.05). Conclusions: The ED RAFF Scale is the first disease-specific QoL measure for use in ED patients with RAFF. It showed good dispersion and stability, whether self- or clinician administered, and good discrimination for the generic QoL SF-8. This scale will be used to judge the success of ED management strategies for RAFF patients. Keywords: atrial fibrillation, quality of life, symptom scores

Jensen, Jan L

jljensen@dal.ca

NO flow time in cardiac arrest management by primary care paramedics using the King laryngeal tube airway versus bag-mask ventilation: a simulation study

Jensen JL, Walker M, Leroux Y, Carter AJE; Emergency Health Services, Dalhousie University, Halifax, NS 
Introduction: Primary care paramedics (PCP) may be the highest trained responders to out-of-hospital cardiac arrest (CA) patients. PCPs traditionally use bag-mask ventilation (BMV) to manage CA patients' airways. In Nova Scotia, the King laryngeal tube (KLT) was added to the PCP scope of practice. The objective of this simulation study was to determine cardiopulmonary resuscitation (CPR) "no flow time" (NFT) during standard CA scenarios with the use of either KLT or BMV by PCPs. Methods: All PCPs were invited to participate at the conclusion of provincial in-service training on KLT. Participants were blinded to outcomes measured. Pairs of PCPs were randomized to KLT or BMV during a standardized 6-minute CA scenario. Scenarios were videotaped, and data abstraction was conducted by one investigator. A $t$-test was used to determine a difference in NFT and time to first ventilation. Results: Sixty-seven pairs of PCPs participated: 30 in the KLT arm and 37 in the BMV arm. Demographics were similar in each study arm: percentage male: KLT 70.0\%, BMV $55.4 \%$; mean age $(95 \% \mathrm{CI})$ : KLT 33.52 (30.44-36.60), BMV 32.07 (29.33-34.81); mean years of experience (95\% CI): KLT 9.03 (6.4911.57), BMV 6.67 (5.16-8.18). Over the 6-minute scenario, mean NFT was significantly improved with KLT 80.50 seconds $(95 \%$ CI 63.07-97.93) versus BMV 110.92 seconds (103.25-118.59). Mean time to first ventilation was longer: KLT 97.73 seconds $(95 \% \mathrm{CI}$ 76.88-118.59), BMV 61.23 (46.75-75.71). The mean time to KLT insertion was 31.83 seconds (26.34-37.32) Conclusion: In this study, NFT was decreased in scenarios managed with the KLT compared to BMV, but time to first ventilation was longer. This small study provides evidence that KLT insertion by PCPs will improve the amount of CPR administered during CA compared to standard basic airway management techniques. Keywords: airway management, emergency medical services, patient simulation

Perry, Jeffrey J

jperry@ohri.ca

THE ADDITION of CT imaging to the ABCD2 score improves the ability to identify individuals at medium and high risk of stroke Jason K. Wasserman, Jeffrey J. Perry, Dar Dowlatshahi, Grant Stotts, Marco L.A. Sivilotti, Andrew Worster, Marcel Émond, Jane Sutherland, Ian G. Stiell, Mukul Sharma; Department of Neurosciences, University of Ottawa, Ottawa, ON

Introduction: The $\mathrm{ABCD} 2$ score to stratify stroke risk after transient ischemic attack (TIA) has low specificity. By adding brain imaging, the proposed $\mathrm{ABCD} 2 \mathrm{I}$ may improve specificity but has not been validated. Our objectives were to (1) validate the ABCD2I score in ED patients with TIA, (2) evaluate the benefit of including only acute CT changes in the ABCD2I, (3) establish a low or high risk score with a similar risk to that proposed by the AHA guidelines. Methods: We prospectively enrolled ED patients with TIA who had a CT scan within 24 hours. Primary outcome was stroke within 2 and 90 days of index TIA. The $\mathrm{ABCD} 2 \mathrm{I}$ score (total 0 to 10 ) was calculated according to Giles et al. A modified $\mathrm{ABCD} 2 \mathrm{Ia}$ was calculated by assigning points for acute infarction. $\mathrm{An} \mathrm{ABCD} 2 \geq 4$ or an $\mathrm{ABCD} 2 \mathrm{I}$ or $\mathrm{ABCD} 2 \mathrm{Ia} \geq 5$ separated lowrisk from medium-high-risk patients. Discriminatory power was calculated from the area under receiver operated characteristic curve (AUC) with $95 \%$ CIs. Results: A total of 2,046 patients were enrolled. The 90day stroke rate was $3.4 \%$. Ischemic changes were present in 565 (27.6\%). The AUCs for the ABCD2, ABCD2I, and ABCD2Ia at 2 days were $0.608(0.514-0.703), 0.601(0.520-0.682)$, and $0.660(0.571-$ 0.748 ), respectively. The AUCs for the ABCD2, ABCD2I, and $\mathrm{ABCD} 2 \mathrm{Ia}$ at 90 days were 0.640 (0.576-0.703), 0.654 (0.596-0.712), and $0.674(0.612-0.735)$, respectively. ABCD2, ABCD2I, and ABCD2Ia identified $61.4 \%, 50.4 \%$, and $38.7 \%$ of patients as mediumhigh risk with an associated 2-day stroke rate of 1.9\% (1.3-2.5\%), $2.0 \%$ (1.3-3.1\%), and 2.5\% (1.6-3.9\%), respectively. ACBD2, ABCD2I, and $\mathrm{ABCD} 2 \mathrm{Ia}$ patients defined as low risk had a $0.9 \%(0.4-1.9 \%), 1.0 \%$
$(0.5-1.8 \%)$, and $0.9 \%(0.5-1.6 \%)$ rate of stroke at 2 days, respectively. Conclusions: The ABCD2Ia score allows separation of patients into those needing urgent evaluation with more specificity than the ABCD2 or ABCD2I. Keywords: computed tomography, risk stratification, transient ischemic attack

\section{Schull, M}

mjs@ices.on.ca

DO clinical decision units improve emergency department patient flow and reduce hospitalizations?

Michael J. Schull, Astrid Guttmann, Marian J. Vermeulen, Chad Leaver, Erin Salkeld, Brian Rowe, Anne Sales; Institute for Clinical Evaluative Sciences, Toronto, ON

Introduction: Clinical decision units (CDU) may improve ED patient flow and hospital operations by reducing hospital admissions. In 2008, Ontario's MoH launched a pilot program and funded seven CDUs. We sought to evaluate the effect of CDUs on ED length of stay (LOS), hospital admissions, and revisit rates. Methods: A retrospective analysis of administrative health data of all ED visits at seven pilot CDU sites and five control sites (controls were eligible for, but did not get, CDU funding) during 18 months of implementation (Oct 2008 to Mar 2010). We modeled outcomes after implementation using GEE for clustering and controlled for age/sex, triage group, teaching status, month, and secular trend. Results: CDU sites admitted $3.9 \%$ of all ED visits to CDUs (site range $2.2-5.6 \%$ ). CDU patients were older than other ED patients (56.7 vs 44.8 years), higher acuity (44.5\% v. $22.2 \%$ CTAS $1-2)$, and higher hospital admission $(20.1 \%$ v. $14.0 \%)$. CDU patients spent more time in the ED than other patients; the median ED LOS, including CDU LOS, was 12.6 v. 3.8 hours. Median LOS in the CDU was 7.1 hours. The most common indications for CDU admission were chest pain $(17.2 \%)$ and abdominal pain $(9.0 \%)$. CDUs were independently associated with small reductions in median ED LOS overall $(-0.1$ hour, $95 \% \mathrm{CI}-0.17,-0.02)$ and for low-acuity patients $(-0.23$ hour, $95 \%$ CI $-0.31,-0.15)$, but a small increase for high-acuity patients $(0.22$ hour, 95\% CI 0.04, 0.40). Discharged patients showed a decrease in median ED LOS $(-0.19$ hour, $95 \%$ CI $-0.24,-0.14)$. Hospital admission rates decreased marginally for moderate-acuity patients $(-0.6 \%$, $95 \%$ CI $-1.1,-0.17)$ with a similar trend for high-acuity patients. No change in ED revisits was seen. Conclusions: About $4 \%$ of ED patients were admitted to CDUs. CDUs were independently associated with small reductions in median ED LOS overall and for lowacuity and nonadmitted patients and small reductions in hospital admission rates for moderate-acuity patients. ED revisit rates were unchanged. This suggests small operational gains for EDs with CDUs. Keywords: administrative database, admission rates, clinical decision units

\section{Perry, Jeffrey J}

jperry@ohri.ca

ANTITHROMBOTIC use in the emergency department in patients with transient ischemic attack

Jeffrey J. Perry, Mukul Sharma, Marco L.A. Sivilotti, Jane Sutherland, Andrew Worster, Grant Stotts, Marcel Émond, Ian G. Stiell; Department of Emergency Medicine, University of Ottawa, Ottawa, ON

Introduction: A study of ED patients with TIA in the province of Ontario in 2000 demonstrated low rates of antithrombotic use, low imaging rates, and a subsequent risk of stroke of $6 \%$ at 90 days. It is unclear whether guidelines or the Canadian Stroke Strategy have impacted the management of TIA patients in the ED. This study assessed antithrombotic medications use, CT neuroimaging, electrocardiogram (ECG) use, and subsequent 90-day stroke rate in a recent cohort of ED patients with TIA. Methods: We conducted a prospec- 
tive cohort study at eight university Canadian EDs over 3.5 years. We enrolled adult ED patients with TIA. Physicians completed data forms prior to discharging or referring patients to consulting services. Patients had 7- and 90-day structured telephone follow-ups with a validated stroke assessment tool. The outcome criterion was stroke assessed by a blinded adjudication committee. Analysis included descriptive statistics. Results: We enrolled 2,775 patients of 3,641 eligible patients $(76.2 \%)$, with mean age 67.8 years (range 19-98), and female $50.2 \% ; 59.1 \%$ history of hypertension, $18.9 \%$ history of ischemic heart disease, and $13.5 \%$ had a previous stroke. At ED discharge, $92.6 \%$ of patients were on antithrombotic medications. ASA was started in $40.2 \%$ of patients, dypyridamole/ASA in $12.8 \%$, clopidogrel for $8.4 \%$, warfarin for $0.9 \%$, and $30.3 \%$ continued existing antithrombotic agent; $96.8 \%$ had a CT in the ED and $91.7 \%$ had an ECG; $9.9 \%$ of patients had ECG findings or history of atrial fibrillation. Only $55.1 \%$ of these patients were discharged on warfarin. The overall rate of stroke was $3.1 \%$ at 90 days. Conclusion: This study demonstrates that subsequent stroke rates in Ontario have declined and that most TIA patients receive $\mathrm{CT}$ imaging in the ED and are managed with antithrombotic agents. Further improvement can be achieved in routinely ordering ECGs and starting patients in atrial fibrillation on warfarin. Keywords: management, prospective cohort, transient ischemic attack

\section{McLeod, Shelley L}

\section{shelley.mcleod@lhsc.on.ca}

*THE IMPACT of AutoPulse on the rate of return of spontaneous circulation in out-of-hospital cardiac arrest in Oxford County

Mal S, McLeod S, Leggett L, Dukelow A, Lewell, M; Division of Emergency Medicine, Faculty of Medicine, The University of Western Ontario, London, ON

Introduction: Automated cardiopulmonary resuscitation (CPR) devices such as AutoPulse allow paramedics to provide high-quality CPR. Oxford County is the first emergency medical services (EMS) system in Canada to outfit each ambulance with such a device. The primary objective of this study was to determine if patients who received CPR with the AutoPulse had a greater rate of return of spontaneous circulation (ROSC) on arrival to the emergency department (ED) compared with those who received traditional CPR. A secondary objective was to determine if there was a difference in survival to hospital discharge between the two groups. Methods: A retrospective medical record review was conducted for all patients in Oxford Country who had an out-of-hospital cardiac arrest during the 2-year study period. A trained data extractor, blinded to the study hypothesis, collected explicitly defined data using a standardized data extraction form. An independent abstractor assessed the reliability in $25 \%$ of randomly selected ambulance call reports (ACRs). Results: A total of 156 consecutive cardiac arrests were reviewed. Of these, $104(66.7 \%)$ patients received manual CPR (m-CPR) and $52(33.3 \%)$ received CPR using AutoPulse (a-CPR). Interrater reliability was $\mathrm{K}=0.983$. A significant difference existed in the primary end point of ROSC between the m-CPR group and the a-CPR group ( 5 of $104[4.8 \%]$ v. 8 of 53 [15.1\%]; $p=.035$ ). However, when survival to hospital discharge was examined, there was no significant difference between the m-CPR and a-CPR groups (4/103 [3.9\%] v. $2 / 51[3.9 \%] ; p=.65)$. We were unable to determine the final outcome in two patients. Conclusions: The use of the AutoPulse in outof-hospital cardiac arrests was associated with higher rates of ROSC. This difference did not translate into a greater proportion of patients surviving to hospital discharge. Further evaluation regarding the potential benefits of the AutoPulse should be performed before this technology is utilized throughout EMS system. Keywords: automated cardiopulmonary resuscitation devices, emergency medical services, medical record review
Rowe, Brian H.

brian.rowe@ualberta.ca

A TAIL of two cities: the role of universal helmet legislation in increasing bicycle helmet use

Rowe BH, Couperthwaite A, Rowe BK, Chetram R, Voaklander DC, Karkhaneh M, Villa-Roel C, Hagel BE; Department of Emergency Medicine and School of Public Health, University of Alberta, Edmonton, AB; Departments of Pediatrics and Community Health Sciences, University of Calgary, Calgary, AB

Introduction: Legislating helmet use has been an injury prevention strategy used by many jurisdictions to increase helmet use and prevent head/brain injuries among cyclists. There is debate regarding the effectiveness of legislation restricted to children compared to all ages (universal). This study evaluated the changes in bicycle helmet use before and after provincial legislation was introduced in 2002 for ages $<18$ years and municipal legislation (all ages) in St. Albert (SA). Methods: Cyclists were observed by trained observers at randomly selected sites in Edmonton (E) and SA from June to October in 2000, 2006, and 2010. Cyclist age $(<18,18+)$, sex, helmet use, helmet compliance, and companions were recorded during fairweather days on commuter routes, bike paths, residential areas, schools/campuses, and in parks. Proportions of helmet use by year (pre [2000] and post [2006, 2010] legislation) for both SA and E are reported. Results: Overall, 7,564 E and 984 SA cyclists were observed at separate locations; more males ( $72 \%$ v. $67 \%)$ and more adults $(68 \%$ v. $47 \%)$ were observed in E. Percentages of helmet use in SA and E were pre- (45\% v. $45 \%$ in 2000$)$ and postlegislation (91\% v. $59 \%$ in $2006,82 \%$ v. $61 \%$ in 2010 ). In 2010 , helmet use was higher in all ages in SA $(80.4 \%$ in children/adolescents and $78.1 \%$ in adults $)$ than in $\mathrm{E}(65.7 \%$ in children/adolescents and $53.4 \%$ in adults). Conclusions: From 2000 to 2010, cycling helmet use increased in both communities. In SA, helmet use increased more dramatically in all age groups following universal legislation. While preliminary, these results suggest that helmet legislation should include all ages. Keywords: bicycle helmets, injury prevention, legislation

\section{Morrison, Laurie}

MorrisonL@smh.ca

EMPLOYING knowledge translation interventions to increase utilization of therapeutic hypothermia post-arrest: the Strategies for Post-Arrest Care (SPARC) Stepped-Wedge Trial

LJ Morrison, P Dorian, KN Dainty, S Brooks, K Thorpe, C Zhan, D Scales; University of Toronto, Medicine, Toronto, ON

Introduction: Current guidelines recommend early institution of therapeutic hypothermia (TH) in survivors of out-of-hospital cardiac arrest (OHCA). However, recent surveys show that TH is delivered inconsistently, incompletely, and with undue delay. Targeted knowledge translation (KT) strategies may increase the proportion of OHCA patients receiving appropriate TH. Methods: We conducted a stepped-wedge cluster randomized trial to evaluate the effectiveness of a multifaceted KT strategy for increasing TH use in a network of 37 hospitals. After a baseline period of 12 months, four wedges of six hospitals were randomized to receive 12 months of passive KT followed by 4 months of active KT. Passive KT consisted of an educational presentation and sharing of a generic protocol and order set. Active KT added a blog, Web-based conferences, annual network events, site visits, and ongoing nurse educator support. The primary outcome was the rate of successful $\mathrm{TH}$, defined as a temperature of 32 to $34^{\circ}$ within 6 hours after emergency department (ED) arrival. Results: During the study, 4,742 OHCA patients were transported to hospital; 1,911 (39\%) achieved ROSC, and 1,063 (22\%) were eligible for TH. Overall, both KT interventions were effective at increasing 
the rate of successful TH, and passive KT led to marked improvements over baseline (96 of 395 v. 30 of 320 patients; OR 2.24, 95\% CI 1.54 to $3.26 ; p<.05)$. Active KT did not improve the primary outcome compared to passive KT ( 86 of 348 patients with active KT; OR $0.94,95 \%$ CI 0.70 to $1.28 ; p=.70)$; however, it did significantly increase rates of initiating TH in the $\operatorname{ED}(p=.04)$. Inappropriate TH remained rare (5-6\% of patients) during both KT phases. Conclusions: A multifaceted $\mathrm{KT}$ intervention markedly improved rates of $\mathrm{TH}$ in a large network of hospitals. Simple passive KT strategies were highly effective in increasing $\mathrm{TH}$ rates, whereas active $\mathrm{KT}$ improved the use of TH in the ED. Keywords: cluster randomized trial, knowledge translation, therapeutic hypothermia

\section{Lee, Patricia}

patriciazlee@gmail.com

REASONS for specialty service consultation delay

Patricia Lee, Brian Rowe, MD, MSc, CCFP(EM), FCCP, Grant Innes, MD, FRCPC, Eric Grafstein, MD, FRCPC, Renee Vilneff, BScN, RN, Eddy Lang, MD, CCFP(EM,) CSPQ; University of Alberta, Edmonton, $\mathrm{AB}$

Introduction: Consultations in the emergency department (ED) are common for patients, especially in high-volume, high-acuity centres. Consultations have been identified as an important contributor to delays in ED throughput and hence crowding. This study examined the reasons for delay for patients experiencing consultant response time (CRT) and decision-making efficiency (DME) delays. Methods: We conducted a prospective cross-sectional study using a representative sample of shifts at three tertiary care centres across the Calgary Health region. Patients over 18 years of age presenting to the ED during sampling periods with a high triage acuity score (Canadian Triage Acuity Scale levels 1-3) were enrolled if the treating emergency physician requested a medical or surgical consultation. We define CRT as the interval from consultant request to arrival and DME from consultant arrival to disposition decision. Predetermined reasons for delay were documented when CRT exceeded 1 hour or DME exceeded 2 hours. Results: Overall, 313 patients were enrolled; 218 consultations (69\%) exceeded either the CRT $(64 \%)$ or DME $(36 \%)$ benchmarks, and $17 \%$ of documented delays exceeded both benchmarks. Overall, 198 (91\%) had a documented reason for consultation delay. The most common reason was "other" $(23 \%)$, which included complex patients, missing collateral information, inappropriate service request, and waiting for new staff turnover. Consultants were also slowed by urgent ward issues $(23 \%)$ or multiple ED consults (22\%). Patient investigations delayed $16.5 \%$ of prolonged consults. Academic teams also hindered the consultation process; $8.6 \%$ of delays were due to a junior member waiting on senior member arrival for disposition. Conclusions: Consultations may be delayed for many and varied reasons. A large percentage of delays can be attributed to a busy consulting service. A possible solution to streamline the consultation process may be to increase staff on busy services based on volume of ward issues and consult requests. Keywords: length of stay, prospective cross-sectional study, specialist consultation

\section{Vaillancourt, Christian}

cvaillancourt@ohri.ca

\section{EFFECTIVENESS of dispatch-assisted CPR instructions: a} prospective multicentre study

Christian Vaillancourt, Ann Kasaboski, Manya Charette, Marianne Hoad, Vivianne Larocque, Denis Crête, Stéphanie Logan, Patrick Lamoureux, Jeff McBride, George A. Wells, Ian G. Stiell; Department of Emergency Medicine, University of Ottawa, Ottawa, ON

Introduction: We sought to determine the frequency of perceived agonal breathing, its impact on the ability of 9-1-1 dispatchers to recognize out-of-hospital cardiac arrest (OOHCA), and the frequency with which dispatch-assisted CPR instructions can be successfully completed. Methods: We conducted a prospective multicentre cohort study of 9-1-1 callers reporting an adult OOHCA patient not witnessed by EMS for whom resuscitation was later attempted in nine cities, with a BLSD/ALS tiered EMS service. We measured patient, caller, and system characteristics during a 22-month period following the introduction of dispatch-assisted CPR instructions. The 9-1-1 dispatchers were trained to use a piloted and standardized data collection tool when listening to 9-11 recordings. We report descriptive statistics. Results: There were 2,316 OOHCA between Jan. 2008 and Oct. 2009. Callers were most often the victim's spouse $(27.8 \%)$ or child $(17.5 \%)$ and were previously trained in CPR (40.6\%). Dispatchers recognized 1,542 of 2,316 (66.6\%) of OOHCA cases; among those not recognized, $n=774,411$ (53.1\%) were still alive early during the call. Agonal breathing was present in 526 of $2,316(22.7 \%)$ of all cardiac arrest cases and accounted for $110 /(774$ 411) $(30.3 \%)$ of missed diagnoses when the victim was not initially alive. CPR instructions were offered to 1,272 of $1,542(82.5 \%)$ and accepted by 514 of $1,272(40.4 \%)$ of callers. Instructions were most commonly not offered because CPR was in progress $(30.0 \%)$ or declined because the caller believed the victim was already dead (36.3\%). Delays occurred between call-to-diagnosis $(2: 13 \mathrm{~min}: \mathrm{sec})$ and during ventilation instructions (3:52min:sec). Chest compression were successfully provided to 325 of 2,316 (14.0\%) additional victims. Conclusions: Dispatch-assisted CPR instruction helped a clinically relevant group of victims who would otherwise not have received CPR. Our findings suggest the need for more education on agonal breathing for dispatchers as proposed by the 2010 resuscitation guidelines. Keywords: cardiopulmonary resuscitation, emergency dispatch, emergency medical services

Rowe, Brian H.

brian.rowe@ualberta.ca

*SEVERE sepsis and septic shock in Canadian emergency departments: a national survey of barriers to evidence-based care

Gaudet J, ${ }^{1}$ Djogovic D, Singh M, Villa-Roel C, Rowe BH; ${ }^{1}$ University of Calgary, Calgary, $\mathrm{AB}$, and University of Alberta, Edmonton, $\mathrm{AB}$

Introduction: Evidence-based guidelines for sepsis are well established, yet many centres are unable to meet quality of care benchmarks. The objective of this study was to determine emergency physicians' opinions of current sepsis clinical practice guidelines (CPGs) and to explore perceived barriers to optimal care in severe sepsis and septic shock in Canadian emergency departments (ED). Methods: A national electronic survey was distributed to CAEP member physicians using a modified Dillman technique. Physicians were asked to complete an anonymous questionnaire about their opinions (Likert scale 1 $=$ strongly agree; $7=$ strongly disagree) regarding sepsis CPGs and the most commonly encountered barriers. Proportions (\%) and median and interquartile ranges (IQR) are reported. A priori academic and nonacademic subgroups were compared using chi-square and Mann-Whitney tests. Results: Of 965 physicians surveyed, 290 (30\%) responded. Most worked in mixed EDs (86\%), seeing a median of 50,000 patients per year, and $52 \%$ worked in an academic/tertiary care ED. Overall, $61 \%$ of physicians reported using a sepsis CPG greater than $50 \%$ of the time to aid their care; however, physicians strongly disagreed that CPGs saved lives in sepsis overall $(7$; IQR 6,7$)$ or when started in the ED (7; IQR 6, 7). Most respondents listed ED overcrowding (66\%), nursing $(66 \%)$ workload, and/or physician $(60 \%)$ workload as the leading barriers to care delivery; there were no differences between academic and nonacademic EDs. ED overcrowding was felt to be the singular most important barrier to implementing current sepsis guidelines (25\%). Conclusions: CAEP physicians report various barriers impeding delivery of care for ED patients with severe sepsis and septic shock. Since workload and ED overcrowding barriers predominate, sepsis care teams may be underutilized. Further research on the utility of sepsis bundles and the efficacy of their specific components could 
help standardize more effective care. Keywords: clinical practice guidelines, sepsis, survey

Campbell, Samuel G

emsgc@cdha.nshealth.ca

THE HALIFAX Procedural Sedation and Analgesia Database: an update

Campbell SG, MacKinley RP, Froese P, Etsell G, LaPierre A, Zed PJ, Butler MB, Magee KD.; Dalhousie University, Halifax, NS

Introduction: Procedural sedation and analgesia (PSA) in the emergency department (ED) at the Halifax Infirmary has been administered by advanced care paramedics (ACP) since 1998. We present data accumulated since our last report in 2005. Methods: A prospective registry of all patients undergoing ACP-administered PSA in our ED between 2006 and 2010. All patients requiring PSA in the ED between 2006 and 2010 had data entered into a pre-established PSA registry. Outcomes included safety assessment and patient satisfaction. Adverse events included oxygen saturation, defined as $<90 \%$ in patients with an initial $\mathrm{SaO}_{2}$ of $>95 \%$, and hypotension, defined as systolic blood pressure $(\mathrm{SBP})<100 \mathrm{~mm} \mathrm{Hg}$ or $\mathrm{SBP}<85 \mathrm{~mm} \mathrm{Hg}$ if initial $\mathrm{SBP}<100$ $\mathrm{mm} \mathrm{Hg}$. Patient satisfaction was determined using a 5-point Likert scale, which included "no recall," "delighted," "satisfied, "unpleasant," and "very unpleasant." Results: The records of 2,320 patients undergoing PSA in the ED were reviewed; $52.9 \%$ were female with an average age of 50.1 years. Most patients were ASA class I (67\%) or II $(27 \%)$. The most common procedures were orthopedic manipulation $(75.4 \%)$, cardioversion $(8.9 \%)$, and abscess incision and drainage $(8.4 \%)$. Propofol was the most common sedative agent utilized $(91.3 \%)$, followed by midazolam $(6.9 \%)$ and ketamine $(0.2 \%)$. Fentanyl was administered to $90.3 \%$ of patients, while $9.7 \%$ received no opioid analgesic. Hypoxia was recorded in 46 patients $(2.0 \%, 95 \% \mathrm{CI}$ 1.4-2.6\%), and an airway repositioning maneuver (jaw thrust) was performed in 371 patients $(16.0 \%, 95 \%$ CI $14.5-17.4)$ of patients. Hypotension occurred in 57 patients $(2.5 \%, 95 \%$ CI $1.8-3.1 \%)$. Overall, $90 \%$ of patients had no recall of the procedure or rated satisfaction as "delighted" or "satisfied." Conclusions: ACP-administered PSA in the ED is performed with very few adverse events and high patient satisfaction. Propofol with fentanyl remains the most commonly administered regimen in our ED. Keywords: advanced care paramedics, procedural sedation, registry

Kanji, Hussein

hdkanji@gmail.com

EMERGENCY cricothyroidotomy: a randomized crossover trial comparing percutaneous techniques: classic Seldinger versus "incision first"

Hussein D. Kanji, MD, MSc, MPH; Warren Thirsk, MD; Sandy Dong, MD, MSc; Michael Szava-Kovats, BSc; Cristina Villa-Roel, MD, MSc; Mira Singh, MS; Brian Rowe, MD, MSc; Department of Emergency Medicine, University of Alberta, Edmonton, AB

Introduction: Emergency cricothyroidotomy is potentially lifesaving in patients with airway compromise who cannot be intubated or ventilated by conventional means. The literature remains divided between the open (surgical) and closed (wire assisted, or Seldinger) techniques as the ideal method. The two are not mutually exclusive, and we hypothesized that an "incision first" (IF) modification to the traditional Seldinger technique may improve the procedure time and success. This study assessed the speed of completion, ease of landmark localization, and success rate of this new IF hybrid technique compared to the Seldinger technique. Methods: A randomized controlled crossover trial with concealed allocation was completed for a total of $180 \mathrm{simu}-$ lated (swine) cricothyroidotomies. Emergency physicians including both staff and residents were enrolled. The primary outcome was time to successful cannulation; secondary outcomes included needle insertion, incision, and dilatation attempts. Finally, proportions of successful intra-tracheal insertion and subjective ease of insertion were compared. Results: The mean time required for the Seldinger technique was $89.3 \pm 5.0$ seconds v. $64.2 \pm 3.9$ for the IF technique $(p<.001)$. The mean numbers of needle insertions ( 1.9 v. $1.2 ; p=.003)$ and dilation attempts $(1.3 \mathrm{v} .1 .0 ; p=.016)$ were higher in the closed technique; there was no significant difference with incision attempts (2.6 v. $2.1 ; p$ $=.08)$. Successful intratracheal insertion was documented in $87 \%$ and $100 \%$ of the closed and IF techniques, respectively $(p=.04)$. All the study participants found the IF hybrid approach easier. Conclusions: The IF modification to the closed technique allows faster and more successful airway access in a validated swine model of cricothyroidotomy. We suggest therefore that the IF technique be considered as an improved method for insertion of an emergency cricothyroidotomy. Keywords: cricothyroidotomy, patient simulation, randomized crossover trial

Payrastre, Julien payrastre@gmail.com

SCRAP the CT chest: a prospective validation pilot study

Julien Payrastre, MD; Andrew Worster, MD; Suneel Upadhye, MD; Daren Lin, MD; Kamyar Kahnamoui, MD; McMaster University, Hamilton, ON

Introduction: The "SCRAP rule" is a five-item clinical decision rule (CDR) designed to identify $100 \%$ of patients with major thoracic injury (MTI) in major blunt trauma without the use of CT that was recently retrospectively derived and validated. The five variables of the SCRAP rule are $\mathrm{SpO}_{2}(>95 \%$ on room air or $98 \%$ on oxygen), chest x-ray, respiratory rate $(<25)$, thoracic auscultation, and thoracic palpation. When all five SCRAP variables are normal, the previous study suggests that patients are at very low risk for MTI and do not require a CT chest. The objectives of this prospective pilot study are to confirm the results of the previous study and to estimate the sample size required for a multicentre prospective validation study. Methods: All trauma patient meeting local trauma team activation criteria from July to August 2010 were included. Patients with penetrating trauma, signs of paralysis, or GCS less than 9 or who were intubated prior to a complete physical assessment were excluded. The study data were prospectively collected prior to obtaining a CT scan on a standardized data collection form. Patients who did not undergo CT were followed up after 10 days in clinic or by phone to ensure that there were no missed MTI. Results: During the study period, the trauma team was activated for 105 patients, of whom 102 were enrolled and 37 met the exclusion criteria. The mean ISS was 11.4. The sensitivity of the SCRAP rule was $100 \%$ (95\% CI $79.1-$ $100 \%$ ); the specificity was $54.3 \%$ (95\% CI $39.2-68.8 \%$ ). No MTIs were missed, and had the rule been applied, three CT scans would have been avoided ( $7 \%$ absolute reduction). Conclusion: The SCRAP rule is $100 \%$ sensitive for MTI in this prospective pilot study. Based on these results, we calculate that 472 patients will need to be enrolled for a future study to have adequate power to validate the SCRAP rule. Keywords: blunt trauma, clinical decision rules, prospective pilot study

Grafstein, Eric

egrafstein@providencehealth.bc.ca

FACTORS affecting regional emergency department selection

Grafstein E, Wilson D, Stenstrom R, Jones C, Tolson M, McKnight D; Department of Emergency Medicine, University of British Columbia, St. Paul's Hospital, Vancouver, BC

Introduction: Increases in regional ED efficiencies might be garnered by shifting patients to less crowded departments. We sought to determine factors associated with a patient's choice of a specific regional emergency department. Methods: We employed a cross-sectional 
face-to-face survey developed using qualitative focus group methodology from 38 recent patient volunteers. The created ED survey was administered to self-directed CTAS 3-5 patients aged $>18$ years presenting to each of six regional EDs in the Vancouver Coastal Health Region from February 25-April 29, 2010. Surveys were conducted 24/7/7. Sample size was calculated based on annual site ED volumes to give a $95 \%$ CI with a precision of $4 \%$ around the primary outcome questions. Results: A total of 634 surveys were completed (SPH 186, VGH 147, RH 100, LGH 92, MSJ 56, UBC 53). Thirty-four percent (217) were CTAS 3. The top factors in the decision to use a specific ED were distance from ED (25.3\%), ED wait times (10.3\%), staff courtesy $(9.5 \%)$, facility reputation $(8.3 \%)$, and perceived quality of care (8\%);57.7\% (95\% CI 53.2-61.2) of patients felt that knowing an ED had a shorter wait time would influence their choice. Multivariable analysis of factors influencing impact of wait times show that having an orthopedic (painful) complaint ( $p=.047$, OR 1.42 [1.05-1.98]) and age $<60(p=.049$, OR $1.47[1.02-2.14])$ created greater importance to wait times. Multivariable analysis of factors associated with choosing distance as important revealed that ED distance $<10 \mathrm{~km}(p=.0011$, OR 2.2 [1.45-3.33]), age $<60(p=.041$, OR 1.58 [1.12-2.26]), and not choosing a downtown $\mathrm{ED}(p=.011$, OR 0.65 [0.46-0.91]) were significant. A total of 458 of $584(78.4 \%)$ respondents said that they would access a website $(95 \% \mathrm{CI}=75.1-81.8 \%)$ to obtain wait time and other ED-specific information. Conclusion: Distance from ED is a more important concern than wait time for self-directed patients choosing a particular ED. Dissemination of ED wait times and other educational material may be used to modulate regional ED visits. Keywords: emergency department selection, patient behaviour, survey

\section{Rowe, Brian $\mathrm{H}$}

\section{brian.rowe@ualberta.ca}

TWEENERS: delays for patients receiveing two emergency department consultations

Rowe BH, Kokotilo A, Brick C, Loewen B, Lowes J, Lovstrom L, Couperthwaite S, Dong K, Lang E, Villa-Roel C; Department of Emergency Medicine and School of Public Health, University of Alberta, Edmonton, AB; Division of Emergency Medicine, University of Calgary, Calgary, $\mathrm{AB}$

Introduction: Emergency department (ED) consultations contribute to prolonged length of stay and ED overcrowding. One special group is those complex patients who do not fit into typical referral groupings and may require more than one consultation. This secondary data analysis was designed to study the flow delays associated with more than one consultation. Methods: Prospective cohort study of convenience shifts from volunteering emergency physicians at two high-volume Edmonton EDs. Physicians provided informed consent and completed standardized consult forms on all patients from whom a consultation was requested from all services except radiology and social services. Administrative data were used to record triage level and times of triage, placement, MD assessment, and consult request. Times of bed request (for admitted) and ED discharge (eg, ward or home) were extracted independently by two reviewers. Continuous data were compared using Mann-Whitney tests, and proportions were compared using chi-square statistics. Results: Overall, 841 patient consults were documented; 93 (11\%) involved patients who required more than one consultation. Patients receiving $>1$ consult were of similar age ( 57 v. $55 ; p=.52)$, sex ( 60 v. $51 \%$ male; $p=.24)$, and documented dementia ( 6.5 v. $3.4 ; p=.14)$ as those who received 1 consultation. Patients with $>1$ consultation experienced longer median LOS (15.9 hours; IQR 8.2, 32.6) than those receiving 1 consultation $(11.2$ hours; IQR 6.7, $22.1-p=.006$ ). The proportion of admissions was higher if $>1$ consultation was requested $(84 \%$ v. $59 \% ; p<.001)$. Conclusions: Consultations involving more than one specialist team constitute $11 \%$ of consults in these EDs and account for considerable delays in dispositions. Efforts to reduce the number of consults per patient, included admission rules, should reduce delays in decision making in the ED. Keywords: length of stay, prospective cohort, specialist consultation

Penciner, Rick

rick.penciner@utoronto.ca

USING a Delphi process to establish a Canadian consensus on emergency medicine clerkship competencies.

Rick Penciner, Trevor Langhan, Richard Lee, Jill McEwen, Rob Woods, Glen Bandiera; Division of Emergency Medicine, Department of Family and Community Medicine, University of Toronto, Toronto, ON

Introduction: Currently, there is no consensus on the core competencies required for emergency medicine (EM) clerkships in Canada. Existing EM curricula have been developed through informal consensus or local efforts. The purpose of this project was to define core competencies for EM clerkships in Canada and to validate a Delphi process in the context of national curriculum development. Methods: Using a modified Delphi process, we developed a consensus among a panel of expert emergency medicine educators from across Canada utilizing the CanMEDS 2005 Physician Competency Framework. A small expert panel (the project investigators, $n=6$ ) developed a comprehensive list of competencies from multiple sources. Using a snowball technique, a larger representative expert panel from across Canada was identified. Using a Web-based survey tool, the expert panel in round 1 rated each competency on a 7-point Likert scale indicating strength of agreement "whether the competency should be included" and a binary scale of "must include" or "not include" in round 2. The competencies were rank-ordered based on \% response of "must include." Competencies ranked by $75 \%$ or more of respondents as "must include" were included in the final list. Upon completion, the project was reviewed by four external reviewers. Results: Thirty experts from nine different medical schools across Canada participated on the panel. The initial list consisted of 152 competencies organized in the seven domains of the CanMEDS 2005 Physician Competency Framework. After the second round of the Delphi process, the list of competencies was reduced to 62 (59\% reduction). The external reviewers commented that the results were valid and applicable to EM clerkships in Canada and that the Delphi methodology was a very effective way to achieve consensus from a large group of educators. Conclusion: This study demonstrated that a modified Delphi process can result in a strong consensus around a realistic number of core competencies for EM clerkships. Keywords: core competencies, Delphi process, medical education

Vaillancourt, Christian cvaillancourt@ohri.ca

CARDIAC arrest diagnostic accuracy of 9-1-1 communication officers: a prospective multicentre study

Christian Vaillancourt, Manya Charette, Ann Kasaboski, Sheldon Cheskes, George A. Wells, Ian G. Stiell; Department of Emergency Medicine, University of Ottawa, Ottawa, ON

Introduction: The frequency with which dispatch-assisted CPR instructions are initiatied inappropriately is rarely reported. We sought to determine the diagnostic accuracy of dispatchers for OOHCA and the frequency with which CPR instructions are initiated for patients not in cardiac arrest. Methods: We conducted a prospective multicentre cohort study of adult OOHCA patients not witnessed by EMS for whom resuscitation was attempted in seven cities, with a BLS-D/ALS tiered EMS service. Dispatchers were laymen and received 6-week training followed by a 6-month preceptorship. Centres prepared deidentified copies of the 9-1-1 recordings every time a cardiac arrest instruction card was followed by a dispatcher, which we reviewed using a piloted standardized data collection sheet. We verified this list 
of cases against a list of prospectively collected OOHCA from a local cardiac arrest registry and submitted a list of missed and required recordings back to the centres. We report descriptive statistics and diagnostic accuracy characteristics with $95 \%$ CI. Results: We analyzed 2,260 recordings between Jan. 2008 and Oct. 2009. Among those, 1,536 were real OOHCA, and 724 were not. Among the 1,536 real OOHCA cases, 1,012 were recognized by the dispatchers (TP) and 524 were not (FN). Among the 724 non-OOHCA cases, dispatchers thought that OOHCA was present in 490 (FP) and absent in 234 (TN). OOHCA diagnostic accuracy characteristics were sensitivity, $65.9 \%$ (95\% CI 63.5-68.2\%); specificity, 32.3\% (95\% CI 29.0-35.9\%); PPV, $67.4 \%$; and NPV, $30.9 \%$. Dispatchers believed that OOHCA was present in 490 of $2,260(21.7 \%)$ cases when it was not, resulting in 54 of $490(11.0 \%)$ patients inappropriately receiving chest compressions, or 54 of 2,260 (2.4\%) of the whole cohort. Conclusions: Dispatchers have a high sensitivity and low specificity for the recognition of OOHCA. We found a very small number of patients receiving CPR when not in cardiac arrest, supporting the current use of dispatchassisted CPR instructions. Keywords: cardiopulmonary resuscitation, diagnostic accuracy, emergency dispatch

\section{Strome, Trevor L}

tstrome@gmail.com

EVALUATION of an emergency department waiting room waittimes display board

Weldon ER, Strome TL, Sustrik R, Serrano N, Pei J; Department of Emergency Medicine, Faculty of Medicine, University of Manitoba, Winnipeg, MB

Introduction: Prolonged emergency department (ED) wait times are a national crisis. The many causes of prolonged time to physician contact have been addressed using multiple quality improvement methods. A novel approach to improve patient satisfaction is to display expected wait times of waiting room (WR) patients. Methods: We describe the utility of a real-time wait-times information board (WTIB) in the waiting room (WR) of a midsized Canadian city community hospital ED. The WTIB displays the number of patients in WR, approximate wait times of WR patients, and estimated time to see a physician. The WTIB derives data directly from the Emergency Department Information System (EDIS). Randomly selected patients in the ED WR were interviewed between June and August 2010 to assess satisfaction with WTIB and ways to improve it. The 18-question survey addressed the usefulness, value, and design of data displayed. Questions were either a 5-point scale, yes/no, or freeform answer. Study results were analyzed descriptively and reported in aggregate. Results: A total of 155 (87\%) patients fully completed the interview. A total of 138 people $(77 \%)$ indicated they had noticed the WTIB while in the WR, and $129(75.4 \%)$ indicated the WTIB was a very valuable or somewhat valuable part of the WR stay; none stated the WTIB was not valuable. Although 77 of 161 (47.8\%) would consider leaving without being seen, 80 of $164(48.7 \%)$ would be willing to wait between 10 and 30 minutes longer than the displayed estimate; 89 (51.2\%) would wait over 30 minutes. The WTIB did not negatively affect LWBS rates. Conclusion: The presence of a waittimes information board is an important innovation for ED waiting rooms provided that the information presented is accurate and meaningful to patients. Results indicate that knowing the expected wait times in an ED waiting room is not likely to increase LWBS rates - a finding supported by actual ED data. The results of this investigation have already been applied to improve the next version of the WTIB. Keywords: information board, patient satisfaction, wait times

McLeod, Shelley L

shelley.mcleod@lhsc.on.ca

PATIENT-related outcomes in suspected renal colic after discharge from the emergency department
Yan JW, Edmonds ML, McLeod SL, Sedran RJ, Theakston KD; Division of Emergency Medicine, Faculty of Medicine, The University of Western Ontario, London, ON

Introduction: Although a relatively benign disease, renal colic can have a significant impact on patient-related outcomes that may not be apparent during an emergency department (ED) visit. The objective of this study was to determine the burden of disease for patients with suspected renal colic after discharge from the ED. Methods: This was a prospective observational cohort study involving all adult ( $\geq 18$ years) patients who presented to the EDs of a tertiary care centre (combined census 150,000 ) with suspected renal colic over a 3-month period (Oct-Dec 2010). Patients were contacted by telephone by trained research personnel at 48-72 hours and 10-14 days after their ED visit to determine use of analgesics, missed days of school or work, and repeat visits to a health care provider. Results: Of 127 patients enrolled, $14(11.0 \%)$ were excluded for definite alternate diagnoses. Of 113 remaining, 8 (7.1\%) were lost to follow-up, leaving $105(92.9 \%)$ providing postdischarge outcomes. Four $(3.5 \%)$ patients were admitted from their initial ED visit. Of those patients contacted, 38 (36.2\%) reported passing a stone within 72 hours and 3 additional patients $(39.0 \%)$ reported passing a stone within 14 days. Eighty-four $(80.0 \%)$ patients required analgesics for a median (IQR) duration of $4(2,7)$ days. There were $27(25.7 \%)$ patients who still required analgesics at the time of the second callback. Fifty-eight (55.2\%) patients missed days of school or work. Of these patients, the median (IQR) duration of missed days of school or work was $2(1,3)$ days. Forty-four $(41.9 \%)$ patients reported the need to see another physician, $6(13.6 \%)$ returned to the ED, $25(56.8 \%)$ saw their family doctor, $3(6.8 \%)$ saw their family doctor and came back to the ED, $1(2.3 \%)$ went to a walk-in clinic, and $9(20.4 \%)$ saw a urologist within 10-14 days. Conclusion: Despite being a non-life-threatening illness, the burden of disease of renal colic is significant. The adverse outcomes of renal colic affect patients and the health care system beyond what may be seen with an isolated ED visit. Keywords: patient-related outcomes, prospective cohort, renal colic

Borgundvaag, Bjug

b.borgundvaag@utoronto.ca

PREVALENCE of CA-MRSA in purulent skin and soft tissue infections in patients presenting to Canadian emergency departments

Borgundvaag B, Ng W, Willey B, Louie L, Wong H, and Katz K, on behalf of the EMERGENT Working Group; Departments of Family and Community Medicine, Medicine, and Microbiology, University of Toronto, Toronto, ON

Introduction: Methicillin-resistant Staphylococcus aureus (MRSA) has emerged as a community pathogen, with community-associated strains harbouring unique genetic characteristics, including the staphylococcal chromosomal cassette mec type IV or V, the Panton-Valentine leukocidin $(p v l)$ gene, and increased susceptibility to non-beta-lactam antibiotics than hospital-associated MRSA. We assessed the prevalence, risk factors, microbiologic characteristics, and outcomes of MRSA from patients presenting to emergency departments across Canada with purulent skin and soft tissue infections (SSTIs). Methods: Between July 1/08 and April 30/09, wound swabs were collected from patients presenting with SSTIs to 18 hospitals and 2 community health centres in 6 provinces. Patients were managed according to usual practice. Patients with swabs positive for $S$. aureus were eligible and underwent a chart audit, while MRSA-positive patients underwent a structured telephone interview. We used basic descriptive statistics for characteristics and prevalence and chi-square test, Fisher exact test, and Student $t$-test as appropriate to compare frequencies. This study was approved by each local REB. Results: A total of 1,353 patients 
were included, comprising 922 MSSA and 431 MRSA SSTIs, for an overall MRSA prevalence in this population of $32 \%$. We observed significant $(p<.05)$ variation in the prevalence of MRSA, by study site (range 11-100\%). MRSA prevalence in patients presenting with SSTIs was significantly higher in Alberta (50\%) and British Columbia (44\%) than elsewhere $(26 \%, p<.05)$. Conclusions: The overall Canadian MRSA prevalence in patients presenting with purulent SSTIs to EDs is strikingly high. The wide variation in prevalence highlights that CAMRSA does not emerge uniformly, making ongoing surveillance important. Keywords: methicillin-resistant Staphylococcus aureus, prevalence, regional variation

Stefan, Raluca Alexandra alexandra.stefan@utoronto.ca

THE EFFECT of point-of-care video tutorials on procedural skill teaching in the emergency department

Atzema, Clare MD, MSc, FRCPC; Stefan, Raluca Alexandra MD, MSc, FRCPC; Saskin, Refik MSc; Michlik, Greg; Austin, Peter, PhD; Sunnybrook Health Sciences Centre and Institute for Clinical Evaluative Sciences, University of Toronto, Toronto, ON

Introduction: The emergency department (ED) is a common setting where trainees learn to perform medical procedures. The real-time use of innovative educational tools such as procedure videos has not been studied in this setting. Our study researched whether viewing a video prior to procedure performance improves the quality of teaching of procedural skills overall and during times of ED crowding. Methods: This is a single-centre, prospective, before and after study. Standardized data collection forms were completed by trainees and supervising emergency physicians (EPs) after each shift in the before and after phase. Online procedure videos were introduced on ED computers in the after phase. The primary outcome measure was EP rating of the quality of teaching provided for the procedure using a 5-point Likert scale. Secondary outcome measures included trainee rating of teaching and EP rating of trainee procedural skill. The interaction between crowding and videos was assessed for each outcome to determine whether videos provide a specific benefit during ED crowding. Results: There were 697 procedures performed by 192 trainees, supervised by 38 EPs. Median number of procedures performed per shift was 1.0 (IQR 0-2.0). Mean EP rating of teaching provided was significantly higher in the group that viewed videos, at 4.2 versus 3.7 ( $p<$ $.001)$. In the adjusted analysis, EP ratings increased by 0.6 with a video $(p<.001)$, and odds of a score 5.0 were greater if a video was viewed (OR 2.20, $p=.03$ ). There was no statistically significant difference in scores for trainee rating of teaching or EP rating of trainee skill when a video was viewed. The interaction of crowding and videos showed a small benefit in EP rating of teaching (0.24) and of trainee skill $(0.33)$ Conclusions: Use of procedural videos was associated with improved EP perception of the quality of teaching provided around procedures. Keywords: before/after study, medical education, video tutorials

\section{Schull, M}

\section{mjs@ices.on.ca}

WAITING with an emergency: short-term mortality and hospital admission following departure from crowded emergency departments

Astrid Guttmann, Michael J. Schull, Marian J. Vermeulen, Therese Stukel; Institute for Clinical Evaluative Sciences, Toronto, ON

Introduction: Emergency department (ED) crowding leads to longer length of stay (LOS), delayed care, and leaving without being seen (LWBS). Patient safety initiatives have focused on reducing delays for time-sensitive treatments in acutely ill patients and on reducing LWBS, thought to represent high-risk groups. Yet most ED patients are seen by a physician and discharged (SAD). Our objectives were to deter- mine among nonadmitted ED patients whether presenting during a crowded shift or LWBS is associated with the risk of death or hospitalization within 7 days of ED departure. Methods: Observational, population-based, health administrative database study to identify nonadmitted ED patients in 2003-2008 in Ontario, Canada. For each patient, we determined departure status (SAD v. LWBS) and shift crowdedness (calculated as the mean ED-LOS among all patients in the same $\mathrm{ED} /$ day/shift/triage-acuity group). Covariates included age/sex, chief complaint, prior ED visits, SES, hospital type, and day/time. We used GEE to account for clustering with the patient as unit of analysis. Results: There were 13,934,542 SAD and 617,011 LWBS patients. In high-acuity patients, the crude rate of 7-day death or hospitalization increased from $2.3 \%$ to $3.1 \%$ for patients seen during shifts with a mean ED LOS $<1$ hour v. $6+$ hours, respectively; the respective increase in low-acuity patients was from 0.7 to $1.3 \%$. The risk increased with increasing shift crowdedness (high acuity adjusted odds ratio [aOR] death, 1.48, 95\% CI 1.13-1.95, aOR hospitalization, 1.75 95\% CI 1.62-1.89; low-acuity aOR death, 2.11 , 95\% CI $1.45-3.08$, aOR hospitalization, $1.8695 \%$ CI 1.74-2.00) for mean EDLOS $6+\mathrm{v}$. $<$ 1 hour. LWBS was not associated with higher risk of adverse events. Conclusions: ED crowding is associated with the risk of death and hospitalization in patients well enough to leave the ED. LWBS patients are not at higher risk of adverse events. Patient safety efforts should target overall ED-LOS reductions since the adverse effects extend to patients thought of as low-risk. Keywords: administrative database, length of stay, patient safety

Calder, Lisa

lcalder@sympatico.ca

ANALYSIS of adverse events in patients presenting to the emergency department with acute respiratory distress

Austin Gagné, Ian G. Stiell, Alan Forster, Lisa A. Calder; Department of Emergency Medicine, University of Ottawa, Ottawa, ON

Introduction: We have previously shown that patients discharged from high-acuity areas of the emergency department (ED) are at increased risk of adverse events (AEs: adverse outcomes associated with health care management). The objective of this study was to determine the preventability, type, and severity of AEs among ED patients discharged with congestive heart failure (CHF), chronic obstructive pulmonary disease (COPD), and community-acquired pneumonia (CAP). Methods: We conducted a structured AE analysis of a prospective cohort of patients 50 years or older discharged from the ED with CHF, COPD, or CAP at two tertiary care EDs. A trained medical student abstracted predefined variables from charts and constructed case summaries. We searched for all patients discharged from the ED who had flagged outcomes (death, admission or return to ED within 14 days post index visit). $\mathrm{AE}$ determinations were made by a trained emergency physician reviewer, using a structured, piloted process. Data were analyzed with descriptive statistics. Results: Of 1,200 enrolled patients, there were 68 (5.7\%) flagged outcomes and 20 adverse events (1.7\%, 95\% CI 1.1-2.3). There were $47.1 \%(N=32$ of 68$)$ female patients and a mean age of 73.8 (SD 9.5). Adverse events occurred among 9 patients with COPD, 8 with $\mathrm{CHF}$, and 3 with CAP. The majority of AEs were preventable $(N=18$ of $20,90.0 \%)$. The two most significant types of preventable AEs were unsafe disposition decisions $(N=9$ of 18$)$ and diagnostic error $(N=8$ of 18). One unsafe disposition was a CHF patient sent home despite an ambulatory oxygen saturation less than $90 \%$. In terms of severity among the AEs, there were 2 deaths, 13 admissions, and 5 patients who returned to the ED. Conclusions: Patients with CHF, COPD, and CAP experience a high percentage of preventable AEs, of which an unsafe disposition decision or diagnostic error was the primary cause. This suggests a need for improved emergent diagnosis and disposition decision making for these patients. Keywords: acute respiratory distress, adverse events, prospective cohort 
Upadhye, Suneel

suneel_upadhye@hotmail.com

PROGNOSTIC validity of the Mortality in ED Sepsis (MEDS) score in ED septic patients: a systematic review

Massoud Jalayer, MD; Suneel Upadhye, MD, MSc

McMaster University, Hamilton, ON

Introduction: The Mortality in ED Sepsis (MEDS) stratified scoring system has been validated in a number of ED settings to prognosticate in-hospital 28-day mortality in ED septic patients. We sought to review and collate these studies and generate pooled mortality strata for the MEDS score. Methods: We completed an electronic search of the MEDLINE, EMBASE, CINAHL, Web of Science, Cochrane, and Lilacs databases, in addition to hand searches of conference proceedings from leading EM/critical care organizations, as well as NLM Gateway and BioMed Central for the same. Hand searches of retrieved articles and bibliographic reviews were also conducted. Data for mortality were extracted for each point stratum where available, and average mortality (range) for each stratum was calculated. The AUC for each study and mean (range) for each stratum were also determined. Results: The search yielded 111 potential studies for screening, of which 15 were included after duplicated searches $(n=$ 11,707 patients). MEDS score mean mortality (range) by strata was as follows: $0-4$ points $=1.65 \%(0.5 .2) ; 5-7$ points $=10.09 \%(2.0-27.5)$; $8-12$ points $=22.08 \%(3.5-41.7) ; 13-15$ points $=23.28(6.7-47) ;$ and $>15$ points $=42.58 \%(10.5-76)$. The mean AUC was $0.78(95 \% \mathrm{CI}$ 0.70-0.82) for the overall score. Conclusions: The MEDS score is a valid risk stratification tool for predicting in-hospital mortality for patients admitted to the ED with sepsis. This validation review elevates the MEDS score to a level II clinical decision rule. Future implementation studies of MEDS-directed patient care determinations and clinical/resource utilization outcomes would likely elevate MEDS to a level I clinical decision rule. Keywords: risk stratification score, sepsis, systematic review

\section{Scheuermeyer, Frank}

frankscheuermeyer@yahoo.ca

PROSPECTIVE evaluation of the adverse event profile of two medication regimens for emergency department procedural sedation and analgesia in elderly patients: a 1:1 mixture of ketaminepropofol ("ketofol") versus propofol

Andolfatto G, Scheuermeyer F, Willman EV, MacManus D, Grafstein E; St Paul's Hospital and the University of British Columbia, Vancouver, $\mathrm{BC}$

Background: Emergency department (ED) patients older than 75 years have differing physiology requirements that may put them at higher risk for adverse events (AEs) for procedural sedation and analgesia (PSA). We sought to compare the safety and efficiency of a 1:1 mixture of ketamine:propofol (KP) with propofol (PR) Methods: As part of a regional safety audit, all patients undergoing PSA at one of three urban EDs from April 1, 2006, to January 31, 2009, were prospectively collected. Consecutive patients $>75$ were evaluated for demographics, comorbidities, vital signs, and ED treatment. Specialized data forms recorded all medications, vital signs, and AEs. Patients received either a 1:1 mixture of ketamine:propofol (KP) or propofol (PR). The primary outcome was the number of AEs; secondary outcome was the recovery time. Data were analyzed by descriptive methods. Results: A total of 320 patients were collected, with 137 in the KP and 183 in the PR group. Mean ages were similar, although the KP cohort had a significantly greater number of females and orthopedic procedures. In the KP group, there were $53 \mathrm{AEs},(38.6 \%, 95 \%$ CI $30.5-46.8 \%)$, while the PR group had 60 AEs $(32.8 \%, 95 \%$ CI $26.0-39.6 \%)(p=.16)$. No AEs resulted in an unplanned admission. In the KP group, median recovery time was 15 minutes (IQR 11-18 minutes); in the PR group, median recovery time was 13 minutes (IQR 9-16 minutes) $(p=.15)$. Conclusions: For procedural sedation in patients $>75$ years of age, both KP and PR appear to be safe and effective. Keywords: adverse events, procedural sedation, prospective cohort

\section{Poster}

Brison, Robert

brisonr@kgh.kari.net

RISK for stroke in patients with pure sensory transient neurologic events

Ramy Kamal, Dr. Jeffrey J. Perry, Dr. Robert J. Brison; Queen's University School of Medicine, Kingston, ON

Introduction: Emergency department (ED) patients with transient neurologic events (TNEs) are generally investigated acutely for cerebral ischemia. We had observed that in those with pure sensory symptoms, these investigations were rarely positive. We wished to assess our hypothesis that pure sensory TNEs are low risk for subsequent stroke. Methods: A nested cohort of patients with pure sensory TNE was identified from a larger research cohort of patients diagnosed with potential transient ischemic attack (TIA) recruited at the Kingston General Hospital ED between January 2008 and January 2010. Information on presenting signs and symptoms had been collected for the full cohort using a standardized data collection form completed by physicians. Those with isolated pure sensory signs and symptoms - defined as the presence of "altered sensation" (ie, numbness, dysesthesia) in the absence of weakness, speech/language/visual disturbance, pronator drift, and/or limb ataxia - were identified using these data forms and the ED medical record. Subjects were followed using hospital records and telephone follow-up for evidence of stroke at 7 and 90 days. Results: The full TIA cohort comprised 274 patients. Of these, 38 (14\%) had pure sensory complaints. Two of these patients had evidence of recent infarction at initial presentation. Both were posterior fossa lesions. No patients had a subsequent stroke within 90 days. Of the remaining 36 patients, the most common final diagnoses provided by consultants were probable TIA (28\%) and migraine variants $(19 \%)$. Conclusions: Pure sensory TNEs are not frequently associated with subsequent stroke and rarely demonstrate infarction on neuroimaging. These findings suggest that most sensory TNEs are not truly ischemic in origin and generally have a low risk for subsequent stroke. These findings, particularly the need to assess for posterior fossa disease, need to be reassessed in a larger study of patients with isolated sensory TNE. Keywords: prognosis, prospective cohort, transient ischemic attack

\section{Vaillancourt, Christian}

cvaillancourt@ohri.ca

IMPACT of dispatch-assisted CPR instructions on bystander CPR and survival rates: a before-after multicentre study

Christian Vaillancourt, Ann Kasaboski, Manya Charette, Stanley Morrow, George A. Wells, Ian G. Stiell

Department of Emergency Medicine, University of Ottawa, Ottawa, $\mathrm{ON}$

Introduction: There currently exists no study powerful enough to conclude in a survival benefit from 9-1-1 dispatch-assisted CPR instructions. We sought to determine the definite impact of such instructions on bystander CPR and survival to hospital discharge rates for adult outof-hospital cardiac arrest (OOHCA) victims. Methods: We conducted the only ethically possible study, a before-after trial enrolling OOHCA patients not witnessed by EMS for whom resuscitation was attempted in seven cities, with a BLS-D/ALS tiered EMS service. We measured victim and system characteristics during a 44-month period before the introduction of dispatch-assisted CPR instructions (April 2004) and dur- 
ing the 35-month period following a study run-in phase to reach our 6,000-case required sample size. Prospective data collection and quality were standardized for all communities. Data analyses include descriptive statistics, attributable risk (AR) with Student $t$-test, adjusted OR with $95 \% \mathrm{CI}$, and time series analyses. Results: There were 2,942 cases between Aug. 1, 2000, and March 30, 2004, and 3,293 cases between Jan. 1, 2007, and Oct. 31, 2009. Victim characteristics were similar in the before and after groups: mean age 68.5 , male $66.9 \%$, witnessed $43.4 \%$, residential location $85.3 \%$, except for initial rhythm VF/VT, which was higher in the before $30.6 \%$ compared to the after $25.0 \%$ group. Bystander CPR rates increased from 15.0 to $28.7 \%$ from the before to the after group (AR 13.7\%; 95\% CI 11.7-15.7\%; $p<.0001$ ). Similarly, survival increased from 4.1 to $5.2 \%$ (AR $1.1 \%$; $95 \%$ CI 0.0 $2.1 \% ; p=.05)$. The adjusted OR of survival as a result of dispatchassisted CPR instructions is 1.45 ; 95\% CI 1.01-2.09. Conclusions: This is the first study powerful enough to determine a bystander CPR and survival to hospital discharge benefit from dispatch-assisted CPR instructions. These results could significantly impact public health policies and the 2015 resuscitation guideline recommendations regarding CPR instructions. Keywords: before-after study, cardiopulmonary resuscitation, medical dispatch

\section{Davis, Matthew}

mdavis57@uwo.ca

THE UTILITY of the prehospital 12-lead electrocardiogram in the emergency department

Matthew T. Davis, Adam Dukelow, Mike Lewell, Shelley McLeod, Severo Rodriguez; Department of Emergency Medicine, Faculty of Medicine, University of Western Ontario, London, ON; Southwest Ontario Regional Base Hospital Program, London, ON

Introduction: Prehospital 12-lead electrocardiogram (pECG) acquisition is becoming an important component of the paramedic scope of practice. Valuable information can be captured with a pECG prior to any prehospital treatments. By the time patients are assessed by an emergency department (ED) physician, their symptoms may have resolved and their initial ED ECG may be normal. Despite the acquisition of the pECG, it is uncertain how many are analyzed by an ED physician. The objectives of this study were to determine how many pECGs are available and/or reviewed by the ED physician and if the pECG has the potential to influence ED management. Methods: A retrospective medical record review was conducted for a random sample of 115 patients 18 years of age or greater who had a pECG between November 1, 2008, and October 31, 2009. All study variables were defined prior to data collection. Data were recorded using a standardized data extraction tool. Prehospital ECGs were independently reviewed by three physicians trained in ECG interpretation. Prehospital ECGs were compared to their first ED ECG to determine if there were any abnormalities not present on the ED ECG. If differences were identified, reviewers determined if it had the potential to change their management. Interrater agreement was measured using kappa $(\kappa)$ statistics. Results: One hundred ten of the 115 charts were included in the analysis. Of the 110 charts reviewed, $47(42.7 \%)$ did not have the pECG included. None of 110 ED charts documented that the pECG was reviewed by the ED physician. Of the 63 pECGs reviewed, 16 (25.4\%) showed changes not apparent on the initial ED ECG $(\kappa=$ $0.83 ; 95 \%$ CI $0.74,0.93)$. Of those, $12(19.0 \%)$ had differences sufficient enough to potentially influence ED management $(\kappa=0.76 ; 95 \%$ CI $0.72,0.82$ ). Conclusions: The pECG has the potential to influence ED management. Improvements in paramedic and emergency physician documentation can be made. A future prospective study is warranted to determine how the pECG influences ED management. Keywords: emergency medical services, medical record review, prehospital electrocardiography
Primiani, Nadia

nadiaprimiani@gmail.com

ARE patients willing and capable of removing their own nonabsorbable sutures?

Peter Macdonald, Nadia Primiani, Adam Lund; Department of Emergency Medicine, Royal Columbian Hospital, New Westminster, BC

Introduction: Providing patients with instructions and equipment regarding self-removal of nonabsorbable sutures could represent a new efficiency in emergency department (ED) practice. Our primary outcome was to determine if patients would be willing and capable of removing their own sutures if provided with suture removal instructions and equipment. Secondary outcomes included complication rates, number of physician visits, time loss from work, and comfort level with suture self-removal. Methods: This prospective, controlled, single-blinded, pseudorandomized trial enrolled consecutive ED patients who met eligibility criteria (age > 19 years, simple lacerations, nonabsorbable sutures). The study group was given wound care instructions, a suture removal kit, and instructions regarding suture self-removal. The control group received wound care instructions alone. Both groups were contacted by telephone at least 14 days after suturing for followup. Secondary questions were addressed at this time. Results: Providing patients with instructions and proper equipment resulted in a significantly higher rate of suture self-removal. In the study group, $91.5 \%$ of patients were able to self-remove their sutures, whereas $64.1 \%$ did so in the control group $(p<.001)$. Study group patients visited their physician less often. Rates of infection, wound dehiscence, and bleeding were similar in both groups and not statistically different. Patients in both groups would be comfortable with suture self-removal in the future. Conclusions: Most patients are willing and capable of suture self-removal. Providing appropriate suture removal instructions and equipment to patients with simple lacerations in the ED appears both safe and acceptable. Keywords: prospective study, suture removal, wound care

\section{Vaillancourt, Christian}

cvaillancourt@ohri.ca

USE of automated external defibrillator by allied health professionals for in-hospital cardiac arrest: a systematic review

Muhammad Tashkandi, Christian Vaillancourt, Sean Moore; Department of Emergency Medicine, University of Ottawa, Ottawa, ON

Introduction: Nurses and allied health professionals are trained and expected to use automated external defibrillators (AED) for cardiac arrests in the community but are seldom authorized to use one during in-hospital cardiac arrests. We sought to determine if implementing an in-hospital AED program could improve time to defibrillation and survival to hospital discharge for cardiac arrest victims. Methods: For this systematic review, we searched MEDLINE, EMBASE, and AMED and reviewed all referenced articles and reviews. Two independent investigators used standardized forms to review papers for inclusion, quality, and data extraction. We included English-language observational studies on the use of AED by allied health personnel for in-hospital cardiac arrest. We calculated kappa interobserver agreement for study selection and evaluated clinical and quality homogeneity. Results: Our search strategy identified 385 papers; 16 were selected for full review (kappa 0.38). All articles selected by at least one reviewer were reviewed in full. Five papers of similar quality ( 1 before-after, four observational studies) met all inclusion criteria (kappa 0.86). One before-after study $(n=561)$ reported that time to first shock did not decrease following the use of AED in patients whose initial rhythm was $\mathrm{VF} / \mathrm{VT}, p=.79$, and was underpowered to find a statistical difference in overall survival to hospital discharge between the AED and the control group (18\% v. 23\%; $p=.09)$. Four observational studies (total $n=509$ ) reported a rate of survival to hos- 
pital discharge in the VF/VT group ranging from 46 to $55 \%$ when an AED was used. The time interval before arrival of the cardiac arrest team ranged from 1 to 5 minutes. In all studies, allied health professionals were nurses. Conclusions: There is a paucity of science pertaining to the use of in-hospital AED by allied health professionals. There exists a time window during which an AED could be applied, and few studies suggest an improvement trend in survival to hospital discharge. Keywords: allied health professionals, automated external defibrillator, systematic review

\section{Lin, Steve}

\section{steven_lin99@hotmail.com}

DROWNINGS in Ontario: an overlooked cause of out-of-hospital cardiac arrests

Jason Buick, BScK; Steve Lin, MDCM; Gerald Kierzek, MD, PhD; Valeria Rac, MD, PhD; Cathy Zhan, MD MSc; Steven Brooks, MD, MHSc, FRCPC; Laurie Morrison, MD, MSc, FRCPC; Rescu, Keenan Research Centre, Li Ka Shing Knowledge Institute, St. Michael's Hospital, University of Toronto, Toronto, ON

Introduction: Drowning is a major public health concern, representing the second leading cause of accidental deaths worldwide with 506 drownings in Canada (2006). Little is known about prehospital characteristics of drowning patients. A better understanding could help prevent these water deaths and guide science, treatment guidelines, and preventive measures. The objective was to investigate prehospital characteristics of drowning patients compared to all cardiac arrests. Methods: A retrospective, observational study of all out-of-hospital cardiac arrest (OHCA) patients of drowning etiology between August 1, 2006, and July 31, 2010, in a Toronto population-based cardiac arrest registry database. All drowning OHCA were eligible. Non-EMS treated cases and EMS witnessed arrests were excluded. Fisher exact test was used to analyze variables. Results: In the study period, 21,014 OHCA were recorded; $114(0.54 \%)$ were attributed to drowning, 82 were treated by EMS, and 32 fit the legislative criteria for obvious death. Most occurred in open water/boat $(46 \%)$ or in residential dwellings $(41 \%)$ Only 2 cases were cooled, and $4 \%$ of cases survived. Approximately $75 \%$ occurred between 8:00 am and 8:00 pm; 49\% occurred in July or August. Drowning cases were younger $(39.5 \pm 24$ v. $65.3 \pm 19$ years, $p$ $<.001)$, unwitnessed $(82.3 \%$ v. $58.3 \%, p<.001)$, and in an initial nonshockable rhythm $(92.7 \%$ v. $78.8 \%, p=.001)$. Differences were not demonstrated for male $(74.4 \%$ v. $65.7 \%, p=.10)$, response time $(6.7 \pm$ 4 v. $7.8 \pm 5.2$ minutes, $p=.07)$, bystander CPR rates $(41.5 \%$ v. $33.8 \%$, $p=.19)$, public access AED application rates (5.06\% v. $2.0 \%, p=.07)$, ROSC $(14.6 \%$ v. $22.9 \%, p=.08)$, and survival to hospital discharge (3.9\% v. $5.11 \%, p=.999)$. Conclusions: Most drownings were unwitnessed OHCA with nonshockable rhythms. This suggests that treatment should focus on early high-quality CPR and prevention. Provincial legislation and public policy should be directed to improve water safety and early initiation of the best possible bystander resuscitation Keywords: administrative database, drowning, emergency medical services

McLeod, Shelley L

shelley.mcleod@lhsc.on.ca

THE INFLUENCE of electronically available wait-time data on choice of emergency department for patients with noncritical medical complaints

Yip A, McLeod SL, McRae A, Xie B; Division of Emergency Medicine, Faculty of Medicine, The University of Western Ontario, London, ON

Introduction: Increased emergency department (ED) wait times lead to more patients who leave without being seen, reduced compliance to discharge instructions, and decreased patient satisfaction. Many EDs post estimated wait times either online or in the ED to guide patient expectations. The objective of this study was to investigate the influence of electronically available wait-time data on patients' choice of ED. Methods: A prospective study was conducted over a 2-month period in the ED of an academic tertiary care centre (annual census 65,000 ). All adult patients ( $\geq 18$ years) assigned a CTAS score of 3,4 , or 5 were approached by trained research assistants to complete a 15item paper-based questionnaire. Patients were excluded if they arrived by ambulance, were cognitively impaired, or had a presenting complaint that was psychiatric in nature. The primary outcomes were awareness of available online ED wait times and willingness to use this information when choosing an ED. Backwards stepwise multivariable logistic regression models determined predictor variables independently associated with the outcomes. Results: A total of 1,211 patients completed the survey. Of these, only $109(9 \%)$ respondents were aware that ED wait-time information was available on the Internet; 544 (44.92\%) reported that they would use available data to make a decision on which ED to visit if they were aware of the information; and $536(44.26 \%)$ indicated that they were more likely to go to the ED with the shorter wait time. Age, gender, household income, education, or Internet access had no influence on awareness of online ED wait times. Younger participants were more likely to utilize the wait-time information if it were made available to them. Conclusion: ED wait-time data available online are not well known to the general public, even after 6 months of the availability of such information. Future work may include a public awareness strategy for ED wait-time data and re-evaluating ED use patterns and patient satisfaction after implementation of that strategy. Keywords: Internet use, patient preferences, wait times

\section{Lee, Patricia}

patriciazlee@gmail.com

EMERGENCY department consultation service performance: measuring responsiveness and decision-making efficiency

Patricia Lee, Brian Rowe, MD, MSc, CCFP(EM), FCCP, Grant Innes, MD, FRCPC, Eric Grafstein, MD, FRCPC, Renee Vilneff, BScN, RN, Eddy Lang, MD, CCFP(EM), CSPQ; University of Alberta, Edmonton, $\mathrm{AB}$

Introduction: Requests for specialty consultation are common for emergency department (ED) patients and often contribute to delays in throughput and increased crowding. Our objective was to quantify the consultation process and describe responsiveness and decision-making attributes of consulting services. Methods: We conducted a prospective cross-sectional study collecting data during a representative sample of shifts at three Calgary tertiary care centres. Patients over the age of 18 presenting to the ED with a high-acuity score (Canadian Triage Acuity Scale levels 1-3) were enrolled if the treating ED physician requested a medical or surgical consultation. Total consultation time (TCT) is defined as the interval from consultation request to disposition decision and consultation impact index (CII) as the percentage of total ED length of stay (LOS) consumed by the TCT. We report consultant response times (CRT - from consultant request to arrival) and decision-making efficiency (DME - from consultant arrival to disposition decision). Performance indices were defined as the percentage of consultations that achieved a CRT and DME of $<90$ minutes. Descriptive statistics using medians and interquartile ranges were used to report all time intervals. Results: For 313 enrolled patients, the median TCT was 139 minutes (IQR 87 to 279 ) with a CII of $18 \%$ (median total ED LOS: 778 minutes: IQR 485 to 1,274). Median CRT was 55 minutes (IQR 21 to 119), and median DME was 57 minutes (IQR 25 to 128). Performance indices for the two most frequently requested inhouse services were $83 \%$ (CRT) and 44\% (DME) for general surgery $(N=53)$, while hospitalist $(N=52)$ rated $55 \%$ and $85 \%$, respectively. Conclusion: The consultation process is highly variable and has an important impact on ED LOS. We describe novel measures related to 
consultation performance that can be used to provide feedback to consulting services and for institutional benchmarking. Keywords: length of stay, prospective cross-sectional study, specialist consultation

Thompson, Graham C

graham.thompson@albertahealthservices.ca

DEVELOPING guidelines for the early recognition and emergency management of severe sepsis in children

Graham Thompson, Samina Ali, Gena Neto, Sarah Mcclennan, Serge Gouin, Allan DeCaen, Anna-Thersa Lobos, Tim Lynch, Jeanette Pearce, Angelo Mikrogianakis, Ari Joffe, Francois Boucher, Alan Drummond, Maala Bhatt, Joe Carcillo, Niranjan Kissoon; Alberta Children's Hospital/University of Calgary, Calgary, AB

Introduction: Severe sepsis in children is a significant burden to families and the health care system, resulting in prolonged hospital stays, massive health care costs, and a mortality rate of $5-9 \%$. It is often underrecognized in children, leading to delayed management and increased adverse outcomes. We provide health care professionals with guidelines that are specifically focused on the early recognition and management of severe sepsis in children aged 3 months to 17 years. Methods: Key stakeholders $(n=16)$, who were selected from a broad base of pediatric, urban and rural emergency medicine, pediatric intensive care, pediatric infectious disease, general pediatrics, family medicine, and nursing professionals, provided expert content. Clinical questions pertaining to early recognition and emergency treatment were developed. A formal literature review including Medline, Embase, CINAHL, and Cochrane databases was performed by two independent university library knowledge services. Evidence was reviewed in subtopic groups. Recommendations were developed according to the GRADE system for quality of evidence. Overall guidelines were created in line with the AGREE instrument assessment tool. Results: Recommendations relating to emergency management of severe sepsis in children were developed. Topics included early identification, vascular access and fluid resuscitation, airway intervention, antimicrobials, blood products, early goal-directed therapy, imaging studies, pain and sedation management, psychosocial supports, steroids, transfer of care, and vasoactive medications. Conclusions: Guidelines for the early recognition and emergency management of severe sepsis in children have been developed for Canadian health care providers. Guideline dissemination and attempts to encourage use will include knowledge translation efforts focusing on multimedia educational platforms and collaboration with national and international organizations. In addition, quality assurance measures to evaluate compliance will be necessary. Keywords: clinical practice guidelines, pediatrics, sepsis

\section{Rowe, Brian $\mathrm{H}$}

brian.rowe@ualberta.ca

SEVERE sepsis and septic shock in Canadian emergency departments: have optimal guidelines translated into optimal care?

Gaudet J,* Djogovic D, Singh M, Villa-Roel C, Rowe BH; *University of Calgary, Calgary, $\mathrm{AB}$, and University of Alberta, Edmonton, $\mathrm{AB}$

Introduction: Proven therapies and evidence-based clinical practice guidelines (CPG) exist for severe sepsis/septic shock management. The objective of this study was to characterize the influence of these advances on the management strategies employed by Canadian emergency department (ED) physicians. Methods: A national electronic survey was distributed to CAEP member physicians using a modified Dillman technique. Physicians were asked to complete an anonymous questionnaire about their management of patients with suspected severe sepsis and septic shock. Statistics are descriptive, and a priori academic and nonacademic subgroups were compared using chi-square tests. Results: Of 965 physicians surveyed, 290
(30\%) responded. Most worked in mixed EDs (86\%), seeing a median of 50,000 patients per year, and 52\% worked in an academic/tertiary care centre. Sixty-one percent of physicians reported using a sepsis CPG $>50 \%$ of the time to aid delivery of care. During the first hour of resuscitation, $86 \%$ of physicians believed $>1 \mathrm{~L}$ of crystalloid was appropriate, and early, empiric administration of antibiotics (while laboratory and radiography results were pending) was reported by $57 \%$ of respondents $>75 \%$ of the time. Blood $(53 \%)$ and Pentaspan (25\%) were the most commonly used colloids; however, colloid use was low overall $(78 \%$ respondents reported that they used colloid $<25 \%$ of the time). Use of vasopressor agents was similar between subgroups, with norepinephrine and dopamine relied on most commonly for patients with persistent hypotension. Only $16 \%$ of physicians reported commonly ordering replacement corticosteroids for persistently hypotensive patients. Conclusions: Canadian ED physicians report that they provide early aggressive crystalloid resuscitation and empiric antibiotics for patients with suspected severe sepsis and septic shock. Wide practice variation exists in several other aspects of sepsis care. A quality improvement initiative may be one strategy to improve practice variation and evidence-based care in this deadly disease. Keywords: clinical practice guidelines, sepsis, survey

\section{Lang, Eddy $S$}

eddy.lang@albertahealthservices.ca

THE PREHOSPITAL management of acute pain due to trauma: national recommendations developed using the GRADE methodology for evidence-based guidelines

Lang E, Brown K, Weik T, Spaite D, Falck-Ytter Y, Todd K, Dayan P, Gausch-Hill M, Oliver Z, Wright J, Sasson C, Sahni R, Hirshon J, Gotschall C, Dawson D, Howse D, Alcorta R, Hunt R; University of Calgary, Calgary, AB

Introduction: The prehospital management of pain due to traumatic injury is highly variable, determined by locally developed protocols, and frequently suboptimal. We developed a clinical guideline for the management of traumatic pain in both adults and children by Advanced Life Support (ALS) providers in the field using a well-established evidence-based guideline (EBG) framework.Methods: We recruited a multistakeholder panel with expertise in acute pain management, guideline development, and EMS outcomes research. The panel used the Grading of Recommendations Assessment, Development, and Evaluation (GRADE) methodology to guide the process of question formulation, evidence retrieval, appraisal/synthesis, and decisions on recommendations. Panel members received GRADE training and conducted comprehensive literature reviews supported by health information specialists. Panelists then developed evidence profiles around specific clinical questions and drafted graded recommendations. All graded recommendations (strong or weak), with descriptors of evidence quality (high, moderate, low, very low), were incorporated into an algorithm. A meeting was convened to review all prepared material and obtain consensus on recommendations.Results: Three strong and three weak recommendations emerged from the process; with two of the former linked to high- or moderate-quality evidence. Guidance was developed for the evaluation and treatment of pain as a function of transport time interval, use of age-appropriate pain scales, selection and dosing of analgesic agents, and a proposed schedule for reassessment and redosing. Conclusions: We successfully created a clinical guideline and protocol for traumatic pain management in the prehospital setting, developed through a rigorous EBG development process. Future research should define optimal approaches for implementation of the guideline as well as the impact of the protocol on metrics of safety and effectiveness. Keywords: emergency medical services, guideline development, pain management 
Vaillancourt, Christian

cvaillancourt@ohri.ca

FACTORS associated with the successful recognition of agonal breathing and cardiac arrest by 9-1-1 communications officers: a national survey

Christian Vaillancourt, Manya Charette, Jan L. Jensen, Jessica Tweedle, Ann Kasaboski, Jeremy Grimshaw, Jamie Brehaut, Martin H. Osmond, George A. Wells, Ian G. Stiell; Department of Emergency Medicine, University of Ottawa, Ottawa, ON

Introduction: Agonal breathing is often not recognized as a sign of cardiac arrest. We sought to identify the factors associated with successful identification of agonal breathing and out-of-hospital cardiac arrest by 9-1-1 dispatchers. Methods: National paper-based survey among dispatchers from 24 cities in 4 provinces using multiple distribution attempts and a modified Dillman method. We developed the interview guide based on the constructs of the Theory of Planned Behavior (TPB), which elicits salient attitudes, social influences, and control beliefs potentially influencing recognition of agonal breathing and cardiac arrest. The survey instrument was developed after completion of 24 iterative interviews, verified for internal validity, and piloted. Recruitment took place between Sept. and Nov. 2010 until reaching $100 \%$ of the required sample size (400). We measured internal consistency using Cronbach alpha and the effect of TPB constructs and specific beliefs with regression analyses. Results: Demographics of the 404 of 821 respondents (49.2\% response rate): mean age 36.9, $64.9 \%$ women, $71.6 \%$ laymen, $74.1 \%$ full time, $37.2 \%$ for $6-10$ years, 95.8\% gave CPR instructions, $29.1 \%$ did not when agonal breathing was present. Median intention to give CPR instructions when agonal breathing is present was 4.3 of 5 ( 5 highest) and predicted by the following TPB constructs: attitude (AdjOR 2.0; $p<.0001$ ), social influence (AdjOR 1.4; $p<.0001$ ), control beliefs (AdjOR 1.1; $p=.03$ ), and specific beliefs: agonal breathing is a sign of cardiac arrest (OR 1.2; $p$ $<.0001$ ), family of victim wants CPR instructions when agonal breathing is present (OR 1.2; $p<.0001$ ), and agonal breathing is not clearly defined in the protocol (OR 1.1; $p<.0001)$. Conclusions: Educational interventions designed to better understand the significance of agonal breathing and the expectations of victim's family and to clarify the existing CPR instruction protocol are most lilely to increase dispatchassisted CPR instructions and bystander CPR rates. Keywords: cardiopulmonary resuscitation, emergency dispatch, survey

Stiell, Ian G

iharris@ohri.ca

USE of rate control medication before cardioversion of recentonset atrial fibrillation or flutter in the emergency department is associated with reduced success rates

Gabriel E. Blecher, Ian G. Stiell, Catherine M. Clement, Robert J. Brison, Brian H. Rowe, Bjug Borgundvaag, Trevor Langhan, Eddy Lang, Kirk Magee, Rob Stenstrom, Jeffrey J. Perry, David Birnie, George A Wells; Department of Emergency Medicine, University of Ottawa, Ottawa, ON

Introduction: In emergency department (ED) patients with recentonset atrial fibrillation or flutter (RAFF), it is unknown whether ventricular rate control affects the success of electrical or chemical cardioversion. We evaluated the influence of rate control medication use as well as other important clinical variables on the success of cardioversion. Methods: We conducted a cross-sectional survey, by health records review, on an observational cohort of all adult RAFF cases with symptom onset $<48$ hours, who had chemical or electrical cardioversion attempted at eight academic hospital EDs over a 12 month period. Univariate analyses were performed as appropriate to find associations between key predictors, including use of IV rate control with beta-blockers or calcium channel blockers and rhythm control drugs, and conversion to sinus rhythm. Predictor variables were then included in multivariate logistic regression modeling to calculate adjusted odds ratios for successful cardioversion. Results: The 634 study patients had these characteristics: mean age 64.7 , male $56.9 \%$, atrial fibrillation $88.3 \%$, atrial flutter $11.7 \%$. Treatments included IV rate control $54.9 \%$, attempted chemical cardioversion $55.8 \%$ (success $79.1 \%$ ), attempted electrical cardioversion $67.5 \%$ (success $92.8 \%$ ). Factors associated with reduced electrical cardioversion success were use of rate control (OR 0.4; 95\% CI 0.2-0.7), rhythm control medication (OR 0.3; 95\% CI 0.2-0.5), and CHADS2 score > 0 (OR 0.4; 95\% CI 0.2-0.8). Associated with successful chemical cardioversion were female gender (OR 2.3; 95\% CI 1.5-3.7) and use of procainamide (OR 2.3; 95\% CI 1.4-3.7). Conclusions: Pretreatment of RAFF patients with either rate control or rhythm control medication reduced the success of electrical cardioversion. Procainamide use was associated with increased success of chemical cardioversion compared to other drugs. Slowing the ventricular rate does not improve the success of either electrical or chemical cardioversion. Keywords: atrial fibrillation/flutter, medical record review, therapy

Bruder, Eric A

ebruder@cogeco.ca

ETOMIDATE use: Canadian emergency physicians' practice habits and opinions

Adrian Millman, Ian Ball, Stacey Ridi, Eric Bruder; Department of Emergency Medicine, Queen's University, Kingston, ON

Introduction: The use of etomidate for rapid sequence intubation in patients with septic shock is controversial. Its favourable hemodynamic profile is ideal for unstable patients, but etomidate is known to cause adrenal suppression, the consequences of which remain uncertain. This paper describes the opinions and practice habits of Canadian emergency physicians when considering etomidate for RSI. Methods: A survey was disseminated via e-mail to 100 randomly selected CAEP members, and their responses were collected using online survey software. A 5-point Likert scale was used to assess physician familiarity with the current literature, their choice of induction agent given various clinical scenarios, and their opinions on the controversy. Our institutional research ethics board approved this project. Results: Our response rate was $42 \%$. Nineteen percent of respondents were CCFP certified, $42 \%$ were CCFP-EM qualified, and $32 \%$ were FRCPC trained. The majority of respondents performed less than 20 RSIs per year. Forty-eight percent (95\% CI 33$63 \%$ ) believed that etomidate caused adrenal suppression in septic patients. Sixty-four percent (95\% CI 50-79\%) report that they do not coadminister corticosteroids in septic patients receiving etomidate. Forty-three percent (95\% CI $28-58 \%$ ) believed that etomidate's favourable hemodynamic properties outweigh the risks of adrenal suppression. Etomidate is the most popular agent used in trauma $(45 \%)$ and cardiogenic shock $(60 \%)$ patients but is used less frequently in undifferentiated shock (38\% etomidate v. $43 \%$ propofol) or septic shock (19\% etomidate v. $60 \%$ ketamine). Over half $(54 \%$, $95 \%$ CI $40-7 \%$ ) of Canadian emergency physicians believe that the controversy is important. Conclusions: Canadian emergency physicians frequently use etomidate. Physicians agree that the controversy regarding its safety is both unresolved and of significant clinical importance. Practice patterns remain diverse. This research characterizes the opinions of practicing physicians and stresses the need for ongoing research to evaluate etomidate's safety. Keywords: etomidate, rapid sequence intubation, survey

Rowe, Brian $\mathrm{H}$

brian.rowe@ualberta.ca

CONSULTATION delays for admitted and discharged patients in two tertiary care emergency departments 
Rowe BH, Lowes J, Brick C, Kokotilo A, Loewen B, Lovstrom L, Couperthwaite S, Dong K, Lang E, Villa-Roel C; Department of Emergency Medicine and School of Public Health, University of Alberta, Edmonton, AB; Division of Emergency Medicine, University of Calgary, Calgary, $\mathrm{AB}$

Introduction: Emergency department (ED) consultations for specialty services are infrequently studied. The delays associated with consultations may identify factors to mitigate ED overcrowding; this project was designed to study the flow delays associated with consultations. Methods: Prospective cohort study of convenience shifts from volunteering emergency physicians at two high-volume Edmonton EDs. Physicians provided informed consent and completed standardized consult forms on all patients in whom a consultation was requested from all services except radiology and social services. Administrative data were used to record triage level and times of triage, placement, MD assessment, and consult request. Times of bed request (for admitted) and ED discharge (eg, ward or home) were extracted independently by two reviewers. Overall length of stay (LOS) results are presented. Continuous data were compared using Mann-Whitney tests, and proportions were compared using chi-square statistics. Results: Overall, 841 patient consults were documented. Patient characteristics: $433(51.5 \%)$ were male, median age was 56 years (IQR 39, 73), and 32 $(3.8 \%)$ had documented dementia. Median time from triage to bed/chair placement (68 minutes; IQR 21, 173), placement to MD (23 minutes; IQR 7, 48), and MD to consult (2.2 hours; IQR 0.9, 4) accounted for approximately $36 \%$ of the total median LOS (11.3; IQR $6.8,23.2)$. Overall, consult decision time (3.7 hours; IQR 2.1, 6.1) accounted for $33 \%$ of the total median LOS. Admitted patients had longer LOS (13.7 hours; IQR 7.4, 28.5) than discharged patients $(9.4$ hours; IQR 5.9, $15.9-p<.001)$; admitted patients were older (60 v. $50 ; p<.001)$, more often had triage scores of I-II (47 v. 35\%; $p<$ $.001)$, and more often had dementia (5.2 v. $1.5 \% ; p=.004)$. Conclusions: Consultations in these EDs account for nearly $33 \%$ of the delays associated with LOS. Efforts to reduce time to decision making in the ED will require interventions that involve both emergency and consultant physicians. Keywords: length of stay, prospective cohort, specialist consultation

\section{Rowe, Brian $\mathrm{H}$}

\section{brian.rowe@ualberta.ca}

DELAYS for elderly patients undergoing emergency department consultations

Villa-Roel C, Loewen B, Lowes J, Brick C, Kokotilo A, Lovstrom L, Couperthwaite S, Dong K, Lang E, Rowe BH; Department of Emergency Medicine and School of Public Health, University of Alberta, Edmonton, AB; Division of Emergency Medicine, University of Calgary, Calgary, $\mathrm{AB}$

Introduction: Emergency department (ED) consultations for specialty services in elderly patients have been infrequently studied. The delays associated with consultations in the era of ED overcrowding require further study; this secondary data analysis was designed to study the flow delays associated with consultations in older ( $>65$ years) patients. Methods: Prospective cohort study of convenience shifts from volunteering emergency physicians at two high-volume Edmonton EDs. Physicians provided informed consent and completed standardized consult forms on all patients in whom a consultation was requested from all services except radiology and social services. Administrative data were used to record triage level and times of triage, placement, MD assessment, and consult request. Times of bed request (for admitted) and ED discharge (eg, ward or home) were extracted independently by two reviewers. Continuous data were compared using MannWhitney tests and proportions were compared using chi-square statistics. Results: Overall, 841 patient consults were documented; 299
$(36 \%)$ involved patients $>65$ years old; $149(49.8 \%)$ were male. Compared to younger patients, older patients had similar severity of presentations $(p=.29)$; however, had more documented dementia $(9.7 \mathrm{v}$. $0.6 \% ; p<.001)$. The older patients experienced longer median LOS $(15.1$ hours; IQR $17.9,29.4)$ than younger patients $(10.4$ hours; IQR $6.3,19.6 ; p<.001)$; this delay was seen with admitted (17.7 hours; IQR 8.4, 34.3) and discharged patients (10.4 hours; IQR 7.7, 18.9). Conclusions: Consultations involving elderly patients constitute more than a third of consults in these EDs. Efforts to improve the care of elderly patients should explore the reasons for delays in decision making among emergency and consultant physicians. Keywords: elderly patients, prospective cohort, specialist consulation

\section{Nadeau, Carole CN}

carole.nadeau@crchuq.ulaval.ca

RISK factors for return to medical care in children with acute viral bronchiolitis who were discharged from the emergency department (ED)

Dr Chantal Guimont, Annie-Claude D'Anjou inf, Dr Daniel Bernier, Dr Patrick Daigneault, Dr Serge Gouin

Introduction: Acute viral bronchiolitis is the principal cause of lower respiratory tract infections in infants all around the world. Ten percent of Canadian infants are affected yearly, and it represents $60 \%$ of all lower respiratory tract infections during the first year of life. Most infants recover at home; only a small percentage are admitted to the hospital. A third of these infants will return to medical care within 28 days following the diagnosis. The main objective of the present study was to determine which factors (the severity of the disease, patient's characteristics, or treatment received in the emergency department) were associated with return to medical care among infants with acute viral bronchiolitis aged less than 18 months. Methods: The project was an observational prospective study involving children of the emergency departments of the Centre Hospitalier de l'Université Laval (CHUL) and of the Centre Hospitalier Universitaire Sainte-Justine (CHU-SJ). All children receiving a first diagnosis of acute viral bronchiolitis aged less than 18 months, discharged from the ED from October 2007 to April 2008, were eligible to participate. Standardized information was given to the parents to facilitate home recovery. Follow-up phone calls were done 7,14 , and 30 days following recruitment. Results: A total of 241 children with acute viral bronchiolitis 4.2) months. Thirty-four percent of infants were recruited. Mean age at diagnosis was 6.3 (revisited the ED in the 28 days following the diagnosis). The only significant risk factor for revisiting was having received treatment with epinephrine in the ED initial visit $(p=.0272)$. Having a history of allergy or eczema (atopy) was marginally associated with revisiting the $\operatorname{ED}(p=.0587)$. Conclusions: In this study, having received treatment with epinephrine was the only significant risk factor for revisiting the ED among infants with acute viral bronchiolitis who were not admitted. Keywords: acute bronchiolitis, emergency department relapse, prospective cohort

Calder, Lisa

lcalder@sympatico.ca

ADHERENCE to clinical practice guidelines for the emergency management of congestive heart failure, acute exacerbations of chronic obstructive pulmonary disease, and community-acquired pneumonia

Sarah Tierney, Ian G. Stiell, Alan Forster, Lisa A. Calder; Department of Emergency Medicine, University of Ottawa, Ottawa, ON

Introduction: We assessed adherence to clinical practice guidelines for the emergency management of patients with acute exacerbations of chronic obstructive pulmonary disease (AECOPD), acute congestive heart failure (CHF), or community-acquired pneumonia (CAP) who 
returned to the emergency department (ED) within 2 weeks. Methods: We conducted this prospective cohort study in two Canadian tertiary care EDs. We included patients initially discharged from the ED with AECOPD, acute CHF, or CAP who returned within 14 days and were subsequently discharged, admitted, or died at 30 days (flagged outcomes). An expert ED panel derived a list of critical actions based on national and international clinical practice guidelines for each illness population. A trained medical student abstracted charts. We systematically reviewed all flagged outcomes for adverse events (adverse outcomes related to health care provided). We calculated the number of critical actions completed on the index visit. We used descriptive statistics for data analysis. Results: Of 1,200 patients enrolled, 68 had flagged outcomes (33 [48.5\%] AECOPD, 29 [42.6\%] CHF, and 6 [8.8\%] CAP). Mean age was 73.8 (SD 9.5), and 47.1\% were female. The expert panel identified $10 \mathrm{CHF}, 13$ AECOPD, and $10 \mathrm{CAP}$ critical actions. Emergency physicians appropriately completed critical actions for CAP patients $93.3 \%$ of the time (SD 8.2), CHF patients a mean of $90.3 \%$ of the time (SD 7.3), and AECOPD patients $73.4 \%$ of the time (SD 13.9). There were 20 adverse events (1.7\%, 95\% CI 0.9-2.4), and the proportion of adverse events to flagged outcomes was highest among CAP ( 3 of $6,50.0 \%$ ) patients followed by CHF ( 8 of 29 , $27.6 \%$ ) and AECOPD ( 9 of 33, 27.3\%). Greater adherence to critical actions was not associated with less adverse events. Conclusions: We found variation in practice when evaluating treatment of AECOPD, acute $\mathrm{CHF}$, and CAP against clinical practice guidelines. Knowledge translation efforts are needed particularly for the AECOPD population. Keywords: acute respiratory conditions, clinical practice guidelines, practice variation

\section{Strauss, Benjamin J}

ben.strauss@gmail.com

PEDIATRIC clavicular fractures: assessment of fracture patterns and risk factors following nonoperative treatment

Benjamin J. Strauss, BSc; Timothy P. Carey, MD; Jamie A. Seabrook, MA; Rodrick K. Lim, BSc, MD; Schulich School of Medicine and Dentistry at The University of Western Ontario, London, ON

Introduction: Clavicular fractures are the most common pediatric long bone fracture, and while the vast majority heal with supportive treatment, complications such as malunion, nonunion, and refracture do occur and can lead to pain and disability. While many studies have characterized adult complication rates and risk factors, no comparable studies to date have looked at clavicular fractures in the pediatric population. We aimed to identify the radiologic and clinical variables that increase the complication rate of clavicular fractures. Identification of these variables would help emergency department staff identify patients who require more thorough follow-up or immediate surgical intervention. Methods: We analyzed radiographs of 537 clavicular fractures on initial presentation to the Children's Hospital Emergency Department at the London Health Sciences Centre over a 4-year period, collecting data on variables such as displacement, angulation, and comminution, as well as data such as age and gender. We then determined the outcome of each fracture by reviewing patients' charts and through a logistic regression analysis determined the variables associated with complications. Results: Of all the fractures treated supportively (ie, nonoperatively), only $2.5 \%$ resulted in a complication. All complications occurred in male patients. Our analysis determined that patient age was an independent predictor of complications, with each year past zero conferring an $18.1 \%$ increase in risk of complication. Furthermore, completely displaced fractures were shown to increase the odds of complication by a factor of 3.2. Conclusions: Although the overall incidence of complications was low, age, male gender, and complete displacement were all predictors of increased risk of negative outcome. These findings help the emergency practitioner identify a group of high-risk pediatric clavicular fractures in which more thorough follow-up should be considered. Keywords: adverse events, clavicle fracture, pediatrics

Gravel, Jocelyn

graveljocelyn@hotmail.com

PREDICTIVE ability of "smelly urine" for urinary tract infection in 1- to 36-month-old children

Marie Gauthier, Serge Gouin, Véronique Phan, Jocelyn Gravel; Department of Pediatrics, CHU Sainte-Justine, Montreal, QC

Introduction: It is not known whether reporting of smelly urine is useful to make a diagnosis of urinary tract infection (UTI) in young children. Objective: To determine whether parental reporting of smelly urine is associated with UTI in young children. Methods: A prospective cohort study was performed in the ED of pediatric hospital. Children aged between 1 and 36 months who visited the ED from 10 am until $6 \mathrm{pm}$ during weekdays and for whom a urine culture (UC) was prescribed for suspected UTI (ie, unexplained fever, irritability, or vomiting) were eligible. Exclusion criteria were antibiotic use other than for prophylaxis, diabetes or other metabolic disease, ureterostomy or urinary catheter in place. For all participants, a standardized questionnaire was administered to the parents by a research assistant, asking whether their child's urine had been smelly over the last 48 hours. Collection of UC was done as usual in the ED. The primary outcome was a UTI defined as $>100 \times 106$ bacteria $/ \mathrm{mL}$ of a single pathogen from midstream void or $>50 \times 106 / \mathrm{mL}$ of a single pathogen through bladder catheterization or any amount of Gram bacteria from suprapubic aspiration (or $>10 \times 106$ of Gram $+/ \mathrm{mL}$ ). Results: A total of 301 children were initially enrolled, but 56 were excluded a posteriori because UC was not done (10), collected by bag (30), and/or contaminated (20). The mean age of the 245 participants was 13 months, and $57 \%$ were female. A smelly urine was recorded by parents in $92(38 \%)$ children, and UTI criteria were fulfilled in $36(15 \%)$. On logistic regression, smelly urine was associated with the risk of UTI (OR 3.1, $95 \%$ CI 1.5-6.5). Smelly urine showed a sensitivity of $0.61(95 \% \mathrm{CI}$ $0.45-0.75)$ and a specificity of 0.67 (95\% CI $0.60-0.73)$. Conclusion: Smelly urine increases the probability of UTI among young children evaluated for presumed UTI. However, it does not have a sufficiently high specificity or sensitivity to definitively rule in or out the diagnosis of UTI. Keywords: diagnostic test characteristics, prospective cohort, urinary tract infection

Woods, Robert A

rob.woods@usask.ca

THE IMPACT of teaching the RAPID mnemonic at the start of emergency medicine clerkship

Robert A. Woods, Krista Trinder, Marcel D’Eon, John McAleer; University of Saskatchewan, Saskatoon, SK

Introduction: The RAPID mnemonic (resuscitation, analgesia and assessment, patient needs, interventions, disposition) has been previously described as an approach to emergency department patients. It was designed as a mental checklist to help junior trainees focus on all aspects of patient care in a comprehensive and efficient manner, addressing issues in order of priority. Its impact on trainee performance has not been assessed. We set out to see if a pocket card and a 1-hour teaching session on the RAPID mnemonic at the start of the emergency medicine clerkship rotation would improve performance. Methods: Students on their emergency medicine clerkship rotation received or did not receive the teaching intervention on an alternate basis. Students were assessed through daily encounter cards, a case presentation, a selfassessment form, focus groups, a prerotation case, and a short-answer exam with the prerotation case repeated. Results: Forty-two students were enrolled, 21 in each group. Students in the intervention group had a significantly higher improvement in the prerotation case (1.82 v. 0.26 , 
$p=.012)$ and a higher overall exam score $(75.13$ v. $70.42, p=.046)$ Students in the focus groups described the mnemonic as useful, referring to it 2 to 3 times per shift. They felt it helped them to remember about pain management and social issues. It also made them think more closely about why they were ordering tests. Conclusion: Exposure to the RAPID approach to emergency department patients at the start of their emergency medicine rotation improved exam scores in clerkship students, and students found it to be a useful mental checklist for comprehensive care. This is a simple mnemonic to teach to students. Clerkship directors and emergency medicine teachers may choose to use this mnemonic as a teaching tool for their trainees. Keywords: comparative study, medical education, teaching aids

\section{Adams, Cherie-Lee A}

\section{cherieleeadams@hotmail.com}

PREHOSPITAL induction of therapeutic hypothermia: a metaanalysis

Cherie-Lee Adams, Caillin Langmann, Michelle Welsford; Division of Emergency Medicine, McMaster University, Hamilton, ON

Introducton: Mild therapeutic hypothermia (TH) for comatose survivors of cardiac arrest is proven to dramatically improve neurologic outcome when started in hospital, and some data suggest this benefit may decline with increasing time to target temperature. This metaanalysis was designed to determine whether prehospital infusion of cold intravenous crystalloid is an efficacious and safe method of inducing TH. Methods: Trials examining the effects of prehospital administration of cold, intravenous crystalloid on core body temperature as a means of attaining $\mathrm{TH}$ in adults after cardiac arrest were identified through two independent searches of the following databases up to Nov. 2010: Medline, EMBASE, CINAHL-EBSCO, Cochrane Central, and Web of Science. A standardized template was then used to assess methodologic quality and abstract data on temperature at emergency department (ED) admission, change in temperature, volume and type of crystalloid infused, time and rearrest rates in the field, vasopressor, and adverse events. Results: Data from three pilot studies involving 63 patients and four comparison studies involving 495 patients were analyzed. Pooled analysis revealed a mean ED admission temperature of $34.296^{\circ} \mathrm{C}(95 \%$ confidence interval [CI] 33.960, 34.632), a mean temperature decrease of $-1.361^{\circ} \mathrm{C}(95 \% \mathrm{CI}-1.633,-1.088)$ for all data, and $-1.461^{\circ} \mathrm{C}(95 \% \mathrm{CI}-1.770,-1.151)$ when RCT data were examined separately. In trials targeting a specific temperature, a mean of 2,045 $\mathrm{mL}(95 \%$ CI $1,526,2,563)$ of crystalloid was infused. No differences were found for odds of death (odds ratio [OR] 0.978; 95\% CI 0.430 , 2.227), rearrest (OR 1.053; 95\% CI 0.487, 2.277), or vasopressor use in the field (OR 1.331; 95\% CI 0.38, 4.812). Conclusion: Infusion of cold intravenous crystalloid in the field is a safe, feasible means of achieving TH for adults with return of spontaneous circulation after cardiac arrest. Widespread implementation of prehospital TH would provide the data required to demonstrate that this prehospital intervention improves patient outcomes. Keywords: emergency medical services, meta-analysis, therapeutic hypothermia

Strasberg, Michelle Z

michelle.strasberg@utoronto.ca

SHIFT handoff in the emergency department: current practice, barriers, and strategies for improvement

Michelle Z. Strasberg, BSc (Hon), MD, and Christopher J. Denny, BA (Hon), MD, MSc, FRCPC, FACEP; University of Toronto, Division of Emergency Medicine, Sunnybrook Health Sciences Centre, Toronto, $\mathrm{ON}$

Introduction: Shift handoff is recognized as a high-risk event for patients. Techniques to improve handoff have been studied, yet little is known about current practice in Canadian emergency departments.
Handoff is influenced by many factors, including physician experience, structure of the department, interruptions, distractions, volume of patients, shift overlap, and patient population. This study clarifies and compares current practices of physicians, outlines barriers, and suggests improvements for effective and safe processes. Methods: An online survey was sent to 131 emergency medicine physicians within the Greater Toronto Area (GTA) affiliated with the University of Toronto. The survey collected qualitative data on current practice, obstacles to handoff, and strategies for improvement. Results: Response rate was $50 \%$. Pediatric specialists accounted for $25 \%$ of respondents. Adverse outcomes were observed by $98.5 \%$ of physicians, including delayed or wrong diagnosis and treatment, prolonged patient stay, conflict with families, and death. Generally, the outgoing physician decided on a time, and handoff was conducted at a computer terminal, used to refer to patient information. Obstacles to good handoff included lack of detail or clear plan for disposition; physician inexperience, bias, and fatigue; interruptions; and patient factors, including acuity and complexity. Strategies for improvement included bedside rounds, use of a standardized tool, designated time and location, and including nursing staff. Analysis also included a comparison of current practice between adult and pediatric physicians. Conclusions: Among emergency physicians in the GTA, there appeared to be widespread agreement that poor handoffs may result in adverse events. However, this study shows little consensus or standardization exists within departments. Multiple tangible strategies were identified to enhance handoff, including use of a standardized tool and bedside handover. This sets a framework for further studies to improve patient safety during handoff. Keywords: patient safety, shift change, survey

milne, william $\mathrm{k}$

monycon@hurontel.on.ca

EMERGENCY department targeted ultrasound (EDTU) in rural communities

Flynn CJ, Weppler A, Theodoro D, Haney E, and Milne WK; The University of Western Ontario, London, ON, and South Huron Hospital, Exeter, ON

Introduction: The Canadian Association of Emergency Physicians (CAEP) published a position statement in 2006 encouraging immediate access to emergency department targeted ultrasound (EDTU) 24 hours a day, 7 days a week. Barriers to advanced imaging care still exist in many rural hospitals. Our study investigated the current availability of EDTU in rural communities and physicians' ability to use this technology. Methods: A literature review and interviews with rural physicians were conducted in the summer of 2010 to design a questionnaire focusing on EDTU. The survey was then sent electronically or via regular mail in November 2010 to all Ontario physicians self-identified as "rural." Statistical analysis was performed using MedCalc V9.6.0.0. Results: There were 207 rural physicians who responded to the survey (response rate $28.6 \%)$. The demographics of responders were male $(70.9 \%)$, mean age (49), year of graduation (1987), years in practice (21), and years in primary community (16). More than two-thirds of physicians $(69 \%)$ practiced in communities $<10,000$. Nearly three-quarters $(73 \%)$ worked in a rural ED. Almost all (97\%) reported having an US in the hospital. However, only $61 \%$ had an US available in the ED. Less than half $(44 \%)$ knew how to use the US, with most (78\%) citing lack of training. Of those using EDTU, 52\% were using it at least once per shift. The most common reason to use EDTU was to rule out acute aortic aneurysm $(51 \%)$. Three-quarters of rural physicians $(75 \%)$ agree or strongly agree that EDTU is a skill all rural ED physicians should have. Conclusions: Patients in many rural emergency departments do not have immediate access to EDTU as advocated by CAEP. This gap in care needs to be addressed to ensure that all patients, no matter where they live, have access to this proven imaging modality. Keywords: emergency department targeted ultrasound, rural medicine, survey 
Bostwick, Joanna

jo.bostwick@gmail.com

PILOT prospective validation of a clinical decision rule to reduce the use of digital rectal examination in trauma

Joanna Bostwick, MD, and Daren Lin, MD; McMaster University, Hamilton, ON

Introduction: A clinical decision rule (CDR) to reduce the use of digital rectal examination (DRE) in trauma has been previously derived. It consists of three questions: age over 65, abnormal neurologic exam, blood at urethral meatus. The objective of this pilot study is to determine whether prospective validation of this clinical decision rule is feasible. Methods: This was a pilot prospective observational study. A protocol sheet was completed for each patient more than 15 years old with blunt trauma requiring trauma team activation. The result of the CDR was determined prior to the DRE. A positive DRE was defined as loss of rectal tone, rectal blood, high-riding prostate, rectal mucosal injury, or palpable pelvic fracture. The patient was followed for evidence of clinically significant injury spinal cord injury, urethral injury, bowel injury, and pelvic fracture at the time of discharge. Results: Thirty-two patients were eligible for the study. Mean age was 45.1 (SD $3.7)$, and $40 \%$ were female. The CDR was $100 \%$ sensitive $(95 \% \mathrm{CI}$ $46.6 \%, 100 \%)$ and $72.4 \%$ specific ( $95 \%$ CI $66.9 \%, 72.4 \%)$ for predicting a clinically significant injury. The CDR was $100 \%$ sensitive $(95 \%$ CI $35.7 \%, 100 \%)$ and $70 \%$ specific $(95 \%$ CI $65.7 \%, 70 \%)$ for predicting a positive DRE. A future prospective multicentre validation trial will require 1,014 enrolled patients. Conclusions: Adult blunt trauma patients with a normal neurologic exam, no blood at urethral meatus, and less than 65 years old have a low likelihood of true-positive abnormal DRE. This finding requires further validation. Keywords: clinical decision rule, digital rectal examination, pilot study

\section{Colacone, Antoinette}

antoinette.colacone@mcgill.ca

SIMULATION methodology for the emergency department: evaluation and impact of alternative triage process scenarios for reducing patient wait times

Alex Guttman, Vedat Verter, Eli Segal, Antoinette Colacone, Marc Afilalo; Jewish General Hospital, McGill University, Montreal, QC

Introduction: Inefficiencies in emergency department (ED) triage can delay access to medical services and impact satisfaction and quality of care. This study developed an ED simulation triage model and evaluated various managerial scenarios for reducing patient wait times. Methods: A time and motion study was undertaken in 2008 for 15 weeks in the triage area of a university tertiary care hospital ED (annual census of 66,315 visits). Observers recorded triage duration and personnel in triage. In addition, arrival times, sociodemographic, and severity were extracted from administrative databases. ARENA software with added optimization component was used. Results: Data on 3,205 walkin and 537 ambulance patients were collected. Analysis showed significant differences between the average waiting times of the ambulance versus walk-in patients ( 3.8 versus 19.3 minutes). The triage simulation model was validated based on one full-time triage nurse $(\mathrm{RN})$ and a second RN being available for 5 hours per day. Simulated scenarios designed to understand how to best utilize a second full-time RN showed that when one $\mathrm{RN}$ is dedicated to ambulance arrivals and the other to walk-in patients, the ambulance wait times can be reduced to 1.5 minutes, while walk-in patients wait significantly longer, $68.3 \mathrm{~min}-$ utes. A follow-up scenario showed that a quick ( $0.5-1$ minute) pretriage to screen for patients requiring ambulatory care can reduce the walk-in patient wait time to 9 minutes. Lastly, the scenario with the most optimal outcome showed that the shortest wait times are achieved when both RNs are simultaneously responsible for ambulance, walk-in patients, and pretriage (wait times 0.63 minute for ambulances and 2.25 minutes for walk-in patients). Conclusions: Simulation methodology allows for testing of various ED triage process scenarios and makes it possible to evaluate the impact of a number of alternatives without disrupting ED triage operations, thereby facilitating managers' ability to make informed decisions about system operational efficiency. Keywords: simulation methodology, triage, wait times

Ross, Marshall

mros@ucalgary.ca

EMERGENCY physician-performed ultrasound to diagnose cholelithiasis: a systematic review

Marshall Ross, Dr. Michael Brown, Dr. Kyle McLaughlin, Dr. Paul Atkinson, Jenny Thompson, Susan Powelson, Dr. Steve Clark, Dr. Eddy Lang; Univeristy of Calgary, Calgary, AB

Study Objective: We sought to determine the diagnostic test characteristics of bedside emergency physician-performed ultrasound (BEDUS) for cholelithiasis in symptomatic emergency department (ED) patients. Methods: We conducted a search of MEDLINE, EMBASE, the Cochrane Library, bibliographies of previous systematic reviews, and abstracts from major emergency medicine conference proceedings. We included studies that prospectively assessed the diagnostic accuracy of BEDUS for cholelithiasis compared to a criterion reference standard of radiology-performed ultrasound (RADUS), computed tomography $(\mathrm{CT})$, magnetic resonance imaging (MRI), or surgical findings. Two authors independently performed relevance screening of titles and abstracts, extracted data, and performed the quality analysis. Disagreements were resolved by conference between the two reviewers. BEDUS performance was assessed with summary receiver operator characteristics curve (SROC) analysis, with independently pooled sensitivity and specificity values across included studies using the MetaDisc software. Results: The electronic search yielded 917 titles; eight studies met the inclusion criteria, yielding a sample of 710 subjects. All included studies used appropriate selection criteria and reference standard, but only one study reported uninterpretable or indeterminate results. Our pooled estimates for sensitivity and specificity were $89.8 \%$ (95\% CI 86.4 to $92.5 \%$ ) and $88.0 \%$ (95\% CI 83.7 to $91.4 \%$ ), respectively. Conclusion: Our study suggests that in patients presenting to the ED with pain consistent with biliary colic, a positive BEDUS scan may be used to arrange for appropriate outpatient followup if symptoms have resolved. In patients with a low pretest probability, a negative BEDUS scan should prompt the clinician to consider an alternative diagnosis. Keywords: cholelithiasis, emergency physician ultrasonography, systematic review

Petrosoniak, Andrew andrew.petrosoniak@utoronto.ca

ACHIEVING competence in procedural skills: a survey of Canadian emergency medicine residents and program directors

Andrew Petrosoniak, Karen Woolfrey; Royal College Emergency Medicine Residency Program, University of Toronto, Toronto, ON

Introduction: Emergency medicine (EM) residents must achieve competence in many procedural skills. The objective of this study was to describe the current state of procedural skills experience among Canadian EM residents and assess the opinions of EM residency program directors (PD) regarding procedural skills training. Methods: A twopart, national Web-based survey was administered to all 13 Canadian accredited Royal College EM residency training programs using a modified Dillman technique. Part 1 was sent to all EM residents to evaluate their experience and comfort in performing 46 procedural skills. Questions addressed comfort levels and experience for each procedure. Part 2 was sent to all EM residency PDs to assess their opinions regarding the same 46 procedural skills. Respondents were asked about the importance and experience required to achieve competence for each proce- 
dure. Results: Thirteen PDs and 86 residents responded to the survey. FAST ultrasound and adult endotracheal intubation required the most number of completions for competence, 28.0 and 26.8, respectively. This was achieved by all senior-level residents (PGY 4, 5). Over 50\% of residents had never performed four procedures deemed extremely important by PDs (pediatric medical and trauma resuscitation, intraosseous line insertion, and epistaxis posterior pack). Overall, resident procedural experience correlated positively with comfort levels Residents indicated that EM followed by critical care rotations provided most procedural opportunities. However, during EM rotations, 75\% of residents indicated that pressure to see patients affected their opportunities to perform procedures. Conclusion: By the completion of EM training, residents achieve sufficient experience to become competent in most procedures except those involving pediatric patients. Department flow may affect resident opportunities to perform procedures. Consequently, certain essential procedures must be taught and practiced in alternative settings to achieve competence. Keywords: medical education, procedural skills, survey

Grafstein, Eric

egrafstein@providencehealth.bc.ca

CORRELATION between emergency physician and nurse assessment of ED surge

Grafstein E, Stenstrom R, Scheuermeyer F, Harris D, Hunte G, DalyGrafstein D, Halli P; St. Paul's Hospital, Vancouver, BC

Introduction: Intradepartmental surge planning to handle periods where the department is stressed due to space limitations, volume or acuity surges, or manpower issues is important for EDs. We sought to enhance the development of meaningful thresholds for key indicators of department function by correlating real-time nurse (RN) and emergency physician (EP) experience during work. Methods: A cross-sectional survey of EPs, triage RNs, and ED charge nurses working in a urban tertiary care academic ED with an annual census of 67,000 patients was undertaken at all times between August and November 2010. Ten factors associated with a favourable (normal care state) versus an unfavourable (perceived risk to patient safety) impression of ED functioning were evaluated. These factors included time to EP assessment, number of waiting room patients, 1-hour and 2-hour ED arrival volumes, number of housestaff and emergency residents working, less than a full complement of nurses, number of boarded patients, delayed time to obtain bloodwork or imaging, and use of a surge overcapacity protocol (OCP). Analysis was by multivariable logistic regression. Results: A total of 216 surveys were completed (158 EPs, $58 \mathrm{RNs}$ ); $64.4 \%$ of respondents felt that the ED was "favourable" at the time of survey, but RNs were less likely to report this $(67.2 \%$ versus $73.4 \%$ for EPs). Even though $3.3 \%$ of responses determined 1 or more of the 10 factors to be "highly unfavourable" (72 of 2,160), only 1\% (2 of 216) felt the overall state of the department was "highly unfavourable." Multivariable odds ratios associated with an unfavourable ED state were 1.9 for nurse versus EP $(95 \% \mathrm{CI}=0.91-4.6) ; 2.7$ for OCP in previous 2 hours (95\% CI $0.8-9.7)$; and 1.5 for every 10 -minute increase in time to EP (95\% CI 1.21-1.85). Conclusion: We describe a methodology for developing thresholds for surge planning. Time to EP seems to be the most sensitive indicator for overall gestalt of department functioning. Nurses trended toward higher sensitivity to a perceived unfavourable state. Keywords: cross-sectional survey, emergency department surge, surge planning

\section{Joo, Daniel}

dan.joo@gmail.com

DIGITAL photography as an emergency department management tool in soft tissue infection: a randomized trial

Daniel Joo, BSc, MD, PGY-3 CCFP-EM, Adam Lund, BSc, MD, MDE, FRCPC, Kerrie Lewis, LPN, EMR, Yasemin Arikan, MD,
FRCPC, Anton Grunfeld, MD, FRCPC; Department of Emergency Medicine, University of British Columbia, Vancouver, BC

Introduction: Outpatient parenteral antiinfective therapy (OPAT) is an effective and economical treatment for skin and soft tissue infections (SSTI). Current documentation methods include written descriptions and drawings of the infected site that may not adequately communicate clinical status. Digital photographic images may offer a more accurate mode of documentation for SSTI, improve continuity of care, increase physicians' ability to judge progress, and reduce OPAT duration. No previous studies address the utility of photodocumentation (PD) in this setting. Objective: To determine whether PD improves patient- and clinician-oriented outcomes. Methods: Single-blinded, prospective, randomized, controlled trial in the EDs of one community hospital and one tertiary care centre in Greater Vancouver, Canada. Consecutive patients aged $\geq 14$ presenting to the ED with nonnecrotizing SSTI requiring OPAT were randomized to one of two groups. Those in the intervention group were treated with standard of care plus photographic documentation at initial and subsequent emergency physician (EP) assessment, while those in the control group received current standard of care alone. Results: Primary outcome is duration of OPAT. Secondary outcomes include hospital admission rates for inpatient therapy, number of patients requiring reinstitution of OPAT following step-down to oral therapy, plus patient, nurse, and EP satisfaction with the photodocumentation protocol as ascertained by surveys. A total of 500 subjects were randomized to either the intervention $(n=$ $259)$ or control $(n=241)$ arms. Results are currently pending; however, interim analysis suggested no difference in our primary outcome of OPAT duration. EPs find photodocumentation helpful in assessing progress; EPs and patients report improved continuity of care. Conclusions: Photodocumentation seems to augment EP assessment of the infection in patients with SSTI, although there appears to be no difference in OPAT duration. Patients report PD as being good or better than standard care. Keywords: digital photography, randomized controlled trial, soft tissue infections

\section{Perry, Jeffrey J}

jperry@ohri.ca

TRANSIENT isolated dysphasia is associated with a high rate of potential cardiac sources for embolism

Jason K. Wasserman, Jeffrey J. Perry, Dar Dowlatshahi, Grant Stotts, Marco L.A. Sivilotti, Andrew Worster, Marcel Émond, Jane Sutherland, Ian G. Stiell, Mukul Sharma; Department of Neurosciences, University of Ottawa, Ottawa, ON

Introduction: A cardiac source is often implicated in strokes in which the deficit includes aphasia. However, much less is known about the etiology of isolated dysphasia after transient ischemic attack - specifically if these patients are more likely to have a potential cardiac source for embolism than those with other forms of transient ischemic attack. Methods: We prospectively identified a cohort of patients receiving a final diagnosis of TIA. Patients with isolated dysphasia were identified if the clinical record revealed an isolated language deficit, expressive or receptive. Potential cardiac sources for embolism were defined as atrial fibrillation identified on history, ECG, Holter monitor, or thrombus on echocardiography. Proportions were compared using Fisher exact test. Results: A total of 1,369 patients were included in the final analysis; 48 were identified as having isolated dysphasia. There was no difference in the rate of hypertension $(60.7 \%$ v. $70.3, p=.098)$, coronary artery disease $(19.4 \% \mathrm{v}$. $33.3 \%, p=.062)$, history of atrial fibrillation $(8.1 \% \mathrm{v} .14 .6 \%, p=$ $.112)$, diabetes $(20.2 \%$ v. $20.8 \%, p=.856)$, or previous stroke $(14.5 \%$ v. $16.7 \%, p=.676$ ) between the two groups; however, patients with isolated dysphasia were more likely to have congestive heart failure $(2.5 \%$ v. $8.3 \%, p=.038)$. In both groups, the symptoms lasted for 
more than 1 hour $(86.2 \%$ and $87.5 \%, p=.488)$. Patients with isolated dysphasia were twice as likely to have a potential cardiac source of embolism ( $10.0 \%$ v. $20.8 \%, p=.026)$. In contrast, there was no difference between the groups in the rate of any carotid stenosis $(41.1 \% \mathrm{v}$. $41.7 \%, p=.862)$ or severe $(>70 \%)$ stenosis $(4.3 \%$ v. $8.3 \%, p=.259)$. Conclusion: Isolated dysphasia is associated with a high rate of potential cardiac sources of embolism, the most common being atrial fibrillation. The identification of isolated dysphasia is helpful in determining etiology and warrants rapid screening for a cardioembolic source. Keywords: cardioembolic, isolated dysphasia, TIA

\section{McLeod, Shelley L}

\section{shelley.mcleod@lhsc.on.ca}

DOCUMENTATION of pretest probability and risk factors for venous thromboembolism in the emergency department of an academic tertiary care centre

Duwyn R, McLeod SL, Fernandes CMB; Division of Emergency Medicine, Faculty of Medicine, The University of Western Ontario, London, ON

Introduction: Pulmonary emboli (PE) and deep venous thrombi (DVT) are common considerations in the emergency department (ED). Clinical decision tools to guide the use of resources in the investigation of these conditions are dependent on a patient's risk factors and pretest probability. The purpose of this study was to determine the rate of documentation of pretest probability and risk factors for venous thromboembolism (VTE) in the ED of an academic tertiary care centre. Methods: A retrospective medical record review was conducted for adult patients ( $\geq 18$ years) who had a D-dimer test ordered through the ED for the purpose of investigating a possible VTE. The presence or absence of a clear statement of pretest probability for either a DVT or PE was recorded from review of the patient's ED chart. Documentation of individual risk factors (Well's criteria or PERC) was also recorded. Results: A total of 803 D-dimer tests were ordered from the ED for suspected VTE. From these, a random sample of $100 \mathrm{D}$-dimer tests was selected for this study. Of the 100 eligible charts, 91 could be located and fit the inclusion criteria. The first 10 charts were independently reviewed by two trained data abstractors using a data collection tool to determine interrater agreement using Cohen kappa statistic. There was complete agreement $(\kappa=1.0)$. Of the 91 reviewed charts, 81 had a D-dimer test ordered for a possible PE and 10 had a D-dimer test ordered for a possible DVT. Fourteen charts $(15.4 \%)$ had clear documentation of pretest probability in the ED chart. When PE and DVT were analyzed separately, the percentages of charts with clear documentation of pretest probability were $16 \%$ and $10 \%$, respectively. Looking at specific risk factors, for DVT, the average number of Well's criteria documented was 3.2. For PE, risk factors were documented in $2.8 \%$ and $4.5 \%$ of charts for Well's criteria and PERC, respectively. Conclusion: Most patients investigated for VTE did not have clear documentation of either pretest probability or specific risk factors. Keywords: pretest probability, Well's criteria, venous thromboembolism

McLeod, Shelley L

shelley.mcleod@1hsc.on.ca

THE CLINICAL scaphoid fracture: diagnosis and treatment variability in current practice

Ramkissoon S, McLeod SL, Shah A.; Division of Emergency Medicine, Faculty of Medicine, The University of Western Ontario, London, $\mathrm{ON}$

Introduction: There is little consensus on the diagnosis and followup of clinical scaphoid fractures in the emergency department (ED). Diagnostic modalities range from x-ray, CT, MRI, and bone scan to follow-up physical examination with reimaging. Initial treatment also varies, ranging from symptomatic treatment to full immobilization until follow-up. This study investigated the diagnostic and treatment modalities for clinical scaphoid fractures in the ED. Methods: A retrospective chart review was conducted for adult patients ( $\geq 18$ years) presenting to an academic ED over a 1-year period who had an initial diagnosis of clinical scaphoid fracture. Charts were analyzed for initial investigations and treatment in the ED, time to follow-up, final diagnosis, and total immobilization time using a structured data collection tool. Results: A total of 92 charts were reviewed; 56 met study inclusion criteria. Of these clinical scaphoid fractures, $93 \%$ (52 of 56) were immobilized until follow-up visit. Of the patients who were immobilized, the majority were placed in a thumb spica $(79 \%, 41$ of $52)$ or thumb splint $(6 \%, 3$ of 52$)$. Eight $(15 \%)$ were immobilized using an unspecified cast type. Twenty-two (39\%) were arranged to have follow-up scaphoid x-rays, while $21(38 \%)$ had a CT wrist arranged. No patients received MRI, bone scan, or other modalities. The median time to definitive diagnosis if scaphoid $\mathrm{x}$-rays were used was 9.7 days (range 7-14 days) and for CT wrist was 4.6 days (range 0-10 days). Of the 56 charts reviewed, 5 scaphoid fractures were identified (8.9\%), 1 using $\mathrm{x}$-ray and 4 using CT scan as the follow-up modality. There were $7(12 \%)$ significant wrist or carpal bone fractures, all identified with CT scans. Conclusions: Diagnostic and treatment variability of the clinical scaphoid fracture exists among emergency physicians. Immobilization type, time to follow-up, and choice of imaging modality varied considerably. This highlights the need for clinical scaphoid fracture guidelines and eventual consensus among treating physicians. Keywords: diagnostic imaging, scaphoid fractures, variability in practice

\section{Scheuermeyer, Frank}

frankscheuermeyer@yahoo.ca

ONE-YEAR outcomes of patients with atrial fibrillation who have spontaneous cardioversion to normal sinus rhythm

Heslop C, Christenson J, Grafstein E, Hunte G, Harris D, Stenstrom R, Innes G, Scheuermeyer F; St. Paul's Hospital and the University of British Columbia, Vancouver, BC

Objectives: Emergency department (ED) patients with atrial fibrillation (AF) who have spontaneous cardioversion to normal sinus rhythm (SR) have not had their long-term outcomes described. Methods: This was a retrospective cohort study utilizing the ED administrative database of two urban hospitals to identify consecutive patients with a primary discharge diagnosis of ECG-proven atrial fibrillation from April 1, 2006, to March 31, 2009. Patients converting to SR without electrical cardioversion, or oral or intravenous antiarrhythmic use, were identified. Each patient's unique provincial health number was linked to the provincial vital statistics registry, provincial stroke registry, and regional ED database to identify deaths, strokes, and ED revisits within 1 year. Patient comorbidities were obtained by manual chart review. Primary outcome was the combined 1-year rate of stroke or death; secondary outcome was the proportion of patients discharged home. Results: Eighty-five consecutive patients with a primary ED diagnosis of atrial fibrillation and spontaneous conversion to SR were collected, with one death and no strokes $(1.2 \%, 95 \%$ CI $0.0-3.3 \%)$ in the following year. Discharge rate was $95 \%$. Conclusions: In this two-centre cohort of ED patients with AF and spontaneous conversion to SR, there was a low event rate, and the majority of patients were discharged home. Keywords: atrial fibrillation, long-term outcomes, spontaneous cardioversion

Hayward, Melissa

melissa.hayward@medportal.ca

ULTRASOUND technologist impression versus radiologist final report for ultrasounds ordered from the emergency department: are there discrepancies? 
Hayward M, Marshall T, Welsford M, Eby R, Ha M, Pardhan A, Fan J, Sherbino J, Upadhye S, Niglas M, Kuuskne M, Healey A; McMaster University, Hamilton, ON

Introduction: Turn-around time of diagnostic imaging can delay emergency department (ED) patient disposition. Ultrasound is a dynamic modality where real-time interpretation occurs at the bedside. We assessed the frequency and nature of discrepancy between the technologist impression and radiologist final report of ultrasounds ordered from the ED. Methods: Formal ultrasounds ordered from the EDs of four academic teaching hospitals during a 3-month period were retrospectively reviewed. Staff emergency physicians used a standardized abstraction form to examine the technologist impression and radiologist final report for any discrepancy. Reviewers then ranked the clinical significance of each discrepancy using a Likert scale-based questionnaire. One hundred percent of studies received independent duplicate review. A blinded third party resolved cases where only one reviewer detected a discrepancy. Time from last image acquisition to radiology final report publication was also abstracted. Results: Interim analysis of 398 ultrasounds from one ED showed an overall discrepancy rate of 5.8\% $(n=23)$ with good interrater agreement $(\kappa=0.76)$. Using the radiologist report as criterion standard, $52 \%(n=13)$ of discrepancies were false positives and $48 \%(n=12)$ were false negatives. By modality, renal studies had the highest discrepancy rate (5 of 28, 18\%). Obstetric and venous Doppler ultrasounds were frequently ordered but rarely discrepant (1 of 70, 1.4\%, and 0 of $59,0 \%$, respectively). Each discrepancy received two independent clinical significance rankings that were considered separately (total $N=46$ ). Discrepancies were found to be overall "insignificant" or "slightly significant" in $41 \%$ of rankings $(n=19)$ and "very significant" or "extremely significant" in $21 \%(n=12)$. The median time from last image acquisition to publication of the radiologist report was 42 minutes. Conclusions: In this preliminary analysis, $5.8 \%$ of studies had discrepancies, one-fifth of which were clinically significant. Discrepancy rate within specific modalities was highly variable. Keywords: discrepancies in interpretation, medical error, ultrasound

Atkinson, Paul

Paul.Atkinson@HorizonNB.ca

WEATHER factors associated with croup presentation to a pediatric emergency department in a warm temperate climate

Paul Atkinson, Adrian Boyle, Richard Lennon; Saint John Regional Hospital, Saint John, NB; University of Cambridge, Cambridge, United Kingdom; Royal North Shore Hospital, NSW, Australia

Introduction: Croup (acute laryngotracheobronchitis) is a common upper respiratory condition in children. Various factors have been investigated to try to explain the variation in croup presentation at particular times of the year. Several studies have looked at the effect of weather conditions on the incidence of croup in cool climates, with differing conclusions. Overall low daily temperatures and low absolute humidity seem to have a highly significant relationship with croup admissions. In our study of children presenting to a hospital emergency department (ED) in New South Wales, Australia, we asked whether the pattern of croup attendances in a warm temperate climate was associated with changes in weather. Methods: We collected data for the number of daily attendances of all 729 cases leaving the ED with a discharge or admission diagnosis of croup over a 798-day time period. We obtained detailed climatic records from the New South Wales Meteorological Office for the same time period. We performed bivariate analysis using Pearson correlations and multivariate analysis using a linear regression model. Results: A linear regression model that used backward elimination of variables, only one daily variable, ground temperature at $9 \mathrm{am}$, was significantly associated with the number of croup attendances $(-0.2062)$. There was a stronger correlation, 0.426 , between the calculated mean monthly temperature and the monthly number of croup admissions. Discussion: We have shown that even in this milder climate, croup does seem to be disease associated with cooler weather. We are unable to conclude that hospital attendances for croup are caused by changes in temperature as other factors, such as the prevalence of viral illness, also follow a seasonal and therefore temperature-related pattern. Keywords: croup, seasonal variations, weather patterns

\section{Scheuermeyer, Frank}

frankscheuermeyer@yahoo.ca

ONE-YEAR outcomes of patients discharged from the emergency department with an elevated cardiac troponin level

Brunner N, Grafstein E, Ramanathan K, Humphries K, Scheuermeyer F; St. Paul's Hospital and the University of British Columbia, Vancouver, $\mathrm{BC}$

Background: Cardiac troponin elevation portends a worse prognosis in diverse inpatient populations. The significance of troponin elevation in patients discharged from emergency departments (ED) without inpatient admission is not well known. Methods: Consecutive patients discharged from two urban ED between April 1, 2006, and December 31, 2007 , with at least one abnormal cardiac troponin (TP) were compared to a troponin negative (TN) cohort matched for age, sex, and primary discharge diagnosis. Patient characteristics were abstracted from medical records. Outcomes were obtained by linking patients with a regional ED and a provincial vital statistics database and adjusted for the following: estimated glomerular filtration rate (eGFR), do not resuscitate (DNR) status, history of coronary artery disease (CAD), Canadian Triage Acuity Scale, and left ventricular hypertrophy (LVH) on electrocardiogram. The primary outcome was a composite of death or admission to hospital within 1 year or ED revisit within 1 month. Results: The cohort $(N=388)$ consisted of 194 TP and 194 TN. In the univariate analysis, TP patients had a higher rate of the primary outcome (OR 2.7, 95\% CI 1.8-4.1, $p<.001$ ) and all of its components ( $p$ $<.05$ for all). After adjusting for covariates, positive troponin remained an independent predictor of the primary outcome (OR 2.0, 95\% CI $1.3-3.2, p=.002$ ) and inpatient admission at 1 year (OR 2.1, 95\% CI $1.3-3.4, p=.001)$. There was no significant difference in mortality (OR $1.7,95 \%$ CI $0.9-3.5, p=0.13$ ) or ED revisit (OR $1.5,95 \%$ CI $0.9-2.4, p=0.12$ ) after adjustment. Conclusions: A positive troponin on discharge from the ED is an independent marker of risk of admission within 1 year, but not mortality or ED revisit. These findings should be confirmed in larger populations. Keywords: atrial fibrillation, cohort study, troponin

\section{Atrie, Damon}

damonatrie@gmail.com

PERCEIVED barriers to adequate analgesia in a Canadian tertiary care emergency department

Damon Atrie, Andrew Healey, Mark Mensour; McMaster University, Hamilton, ON

Introduction: Pain is a leading reason for visits to the emergency department (ED). However, oligoanalgesia remains a problem in the ED. The purpose of this study was to determine the perceived barriers to adequate analgesia in a tertiary care teaching urban ED system. Methods: A modified Dillman method was utilized to maximize survey response rate. Data were collected anonymously using an electronic 23- to 25-item questionnaire. All Hamilton emergency physicians, ED nurses, and emergency medicine residents $(n=257)$ were asked to rate various barriers as to how often they prevent adequate analgesia. The survey was piloted to a selected group of physicians, nurses, and emergency medicine residents for feedback on design and question clarity. Results: A total of 197 surveys were returned $\mathbf{7 6 . 7 \%}$ response rate). The barriers identified by respondents as presenting the 
largest barriers in the ED were the responsibility of caring for other patients in addition to a patient in pain $(51.5 \%)$, lack of time to adequately assess and control pain (43.3\%), and use of alcohol or recreational drugs $(39 \%)$ (the percentage signifies the percentage of respondents who believe that the barrier is present often or more frequently in their practice). Conclusions: Analgesia is often not seen as a priority in the ED setting. Because of the high prevalence of pain among ED patients, educational programs and analgesia protocols may be of benefit in eliminating oligoanalgesia and reducing time to analgesia in the ED. Keywords: analgesia, barriers, survey

Rowe, Brian $\mathrm{H}$

brian.rowe@ualberta.ca

THE PERFECT storm: increasing bicycle helmet use and compliance following mandatory helmet legislation

Rowe BH, Rowe BK, Chetram R, Couperthwaite A, Voaklander DC, Karkhaneh M, Villa-Roel C, Hagel BE; Department of Emergency Medicine and School of Public Health, University of Alberta, Edmonton, AB; Departments of Pediatrics and Community Health Sciences, University of Calgary, Calgary, $\mathrm{AB}$

Introduction: Despite legislation mandating helmet use in many jurisdictions to prevent head/brain injuries among cyclists, noncompliance with correct helmet wearing recommendation may limit its effectiveness. This study evaluated the influence of helmet use, and compliance before and after legislation targeting those $<18$ years old was introduced in 2002 in Edmonton (E) and St. Albert (SA), Alberta, with SA implementing all-ages legislation in 2006. Methods: Cyclists were observed at randomly selected sites in E and SA from June to October in 2000, 2006, and 2010 by trained observers. Cyclist age $(<$ $18,18+$ ), sex, helmet use, helmet compliance, and companions were recorded by trained observers. Helmet compliance included correct helmet type and position and use of chin straps; more detailed data were collected in 2010. Proportions of helmet use by year (pre [2000] and post [2006, 2010] legislation) are reported. Results: Overall, 8,548 cyclists were observed at separate locations over the three summer study periods; more male (72 v. $70 \%)$ and more adult cyclists $(69$ v. $53 \%$ ) were observed in $2006 / 2010$ when compared to the 2000 baseline. Percentages of helmet use increased from pre (45\% in 2000) to post (62\% in $2006,65 \%$ in 2010$)$ legislation. Helmet compliance was higher following legislation (2000: 50\%; 2006: 91\%; 2010: 91\%; $p<.001)$; however, $321(13 \%)$ riders wore nonbicycle helmets in 2010. In 2010, the most common helmet wearing errors were helmet too far back (40\%) and straps too loose (36\%). Conclusions: Cycling helmet use has increased across the capital region of Alberta in all ages. In addition, helmet compliance has improved. These results suggest that helmet legislation may be effective to increase overall helmet use as well as correct helmet use. Keywords: cycling helmets, legislation, pre/post study

McLeod, Shelley L

shelley.mcleod@1hsc.on.ca

EFFECT of an emergency department sepsis protocol on adherence to early goal-directed principles

Arbeau RP, McLeod SL, Fernandes CMB, Moss M, Doran S, Sedran RJ; Division of Emergency Medicine, Faculty of Medicine, The University of Western Ontario, London, ON

Introduction: The aim of early goal-directed therapy (EGDT) is to reduce the incidence of septic shock-related morbidity and mortality. The objective of this study was to compare sepsis management before and after implementation of an emergency department (ED) sepsis protocol. A secondary objective was to examine if there was improved adherence to selective EGDT principles, including time to antibiotics, lactate, and the capture of complete vital signs. Meth- ods: This was a phased, before-after cohort study at an academic, tertiary care centre (annual ED census 75,000 visits). We collected continuous data on admitted adult ( $\geq 18$ years) ED sepsis patients during the period before (April 1-June 30, 2009) and after (April 1June 30, 2010) adoption of an ED sepsis screening tool. Results: Sixty-eight patients were included. Patients in both phases were comparable in terms of demographics (45.6\% male; mean [SD]; 64.3 [18.4] years). The screening tool correctly identified 38 of 113 $(33.6 \%)$ patients who were screened for severe sepsis or septic. After implementation of the screening tool, $100 \%$ of patients had complete vital signs recorded compared to $56.7 \%$ prior to implementation $(\Delta 43.3 \% ; 95 \%$ CI $24.9 \%, 60.8 \% ; p<.001)$. Lactate was drawn in $97.4 \%$ of patients after adoption of the tool compared to $70 \%$ prior to adoption $(\Delta 27.4 \% ; 95 \%$ CI $10.2 \%, 45.4 \% ; p=.004)$. Prior to implementation, $93.3 \%$ received antibiotics with a median (IQR) time to administration of $131.0(86.5,220.3)$ minutes, compared to $100 \%$ who received antibiotics within $110.5(54.5,228.7)$ minutes once the tool was adopted. Survival to hospital discharge was not statistically different after the implementation of the ED sepsis screening tool (pre $80 \%$ v. post $86.8 ; p=.52$ ). Conclusions: Implementation of an ED sepsis protocol improved adherence to the selected EGDT principles. Although not statistically different, there was a clinically meaningful reduction in time to antibiotics and increase in the survival to hospital discharge for patients with severe sepsis or septic shock. Keywords: early goal-directed therapy, screening, sepsis

Stiell, Ian G

iharris@ohri.ca

RADIATION doses to emergency department patients undergoing computed tomography

James C. Worrall, Sadia Jama, Ian G. Stiell; Department of Emergency Medicine, University of Ottawa, Ottawa, ON

Introduction: Many physicians are concerned about exposing patients to radiation from diagnostic CT scanning, but estimates of radiation doses vary. This study's objective was to determine the doses from CT scanning for common indications in a Canadian emergency department (ED) using modern multidetector CT scanners. Methods: We conducted a health records review of consecutive adult patients seen at a busy tertiary care ED over a 2-month period and who had undergone CT scanning ordered by emergency physicians (EPs). Cases were identified by searching a PACS database. Data collected included patient age and sex, study indication, scanner model, body area, and reported dose-length product (DLP). Effective dose was calculated by multiplying the DLP by a conversion factor specific to the anatomic area of the scan, as described in the radiation dosimetry literature. Data were collected on a standardized data form, entered into and an electronic database, and then analyzed with descriptive statistics and 95\% CIs. Results: During the study period, EPs saw 19,880 patients, and 2,584 (13\%) had CT scans ordered. Study patients had a mean age of 59 years and $55.5 \%$ were female, and in $4.5 \%$ of these encounters, the patients had multiple scans. Of 2,693 scans with complete dose information, the most common studies were simple head in 1,440 (53.5\%), complex head in 47 (1.7\%), cervical spine in $83(3.1 \%)$, CT pulmonary angiogram in $188(7.0 \%)$, abdomen (nontraumatic abdominal pain) in 522 (19.4\%), and abdomen (renal colic) in 195 (7.2\%). Mean doses for common indications were simple head, $2.6 \mathrm{mSv}$; complex head, $6.1 \mathrm{mSv}$; cervical spine, $2.8 \mathrm{mSv}$; CT pulmonary angiogram, $9.4 \mathrm{mSv}$; abdomen (nontraumatic abdominal pain), $16.4 \mathrm{mSv}$; abdomen (renal colic), $11.5 \mathrm{mSv}$. Conclusions: Many ED patients undergo CT scanning with substantial radiation exposure. ED physicians should be aware of typical radiation doses for the studies they order and how the dose varies by protocol/indication. Keywords: CT scanning, health records review, radiation 
McLeod, Shelley L

shelley.mcleod@lhsc.on.ca

A 5-YEAR retrospective review of sepsis in the emergency department

Arbeau RP, McLeod SL, Doran S, Sedran RJ; Division of Emergency Medicine, Faculty of Medicine, The University of Western Ontario, London, ON

Introduction: Resuscitation guided by an early goal-directed therapy (EGDT) protocol has been shown to improve mortality in severe sepsis and septic shock. The "golden hour" of EGDT represents a critical time period when early recognition and management of sepsis have the ability to significantly impact patient outcome. The objective of this study was to determine the adherence to EGDT principles at our institution. Methods: A retrospective medical record review was conducted for all adult ( $\geq 18$ years) patients admitted from the emergency department (ED) of an academic tertiary care centre (annual census 75,000 ) with a primary diagnosis of sepsis from 2005 to 2009. Data were extracted using a standardized data collection tool by trained research personnel. Results: A total of 348 patients were identified; 28 were excluded as direct admissions, 7 charts were unavailable, leaving 313 included in the review. The mean (SD) age was 64.8 (18.8) years, and $53.7 \%$ were male. Most were CTAS $2(51.1 \%)$ or 3 $(39.9 \%)$. Complete vital signs were recorded in $67.1 \%$ of patients in 2009 compared to $44.8 \%$ in $2005(\Delta 22.3 \%$; $95 \%$ CI $1.2 \%, 41.5 \%)$. Over the 5-year study period, antibiotics were given to $317(91.1 \%)$ patients (range 84.9 to $97.1 \%$ ). Median (IQR) time to antibiotics from physician assessment decreased from $112(67,180)$ minutes in 2005 to $90(41,155)$ in 2009 . Survival to hospital discharge improved from $69 \%$ in 2005 to $84.3 \%$ in $2009(\Delta-15.3 \% ; 95 \%$ CI $-34.7 \%, 1.9 \%)$. Conclusions: Over the 5-year study period, significant improvements in the early recognition and management of sepsis were made, specifically in the time from physician assessment to delivery of antibiotics and survival to hospital discharge. However, additional improvements are necessary as $32.9 \%$ of patients did not have complete triage vital signs recorded in 2009 and time to antibiotics exceeded the golden hour that EGDT strives for. Keywords: early goal-directed therapy, screening, sepsis

Scheuermeyer, Frank

frankscheuermeyer@yahoo.ca

EMERGENCY department management and 1-year outcomes of patients with atrial fibrillation

Scheuermeyer F, Christenson J, Innes G, Harris D, Hunte G, Stenstrom R, Poureslami I, Heslop C, Pourvali R, Grafstein E; St. Paul's Hospital and the University of British Columbia, Vancouver, BC

Objectives: Atrial fibrillation (AF) is the most common arrhythmia seen in the emergency department (ED). We describe the management and 1year outcomes of ED patients with AF. Methods: This was a retrospective cohort study utilizing the ED administrative database of two urban hospitals to identify consecutive patients with a primary discharge diagnosis of ECG-proven atrial fibrillation from April 1, 2006, to January 31, 2009. Each patient's unique provincial health number was linked to the provincial vital statistics registry, provincial stroke registry, and regional ED database to identify deaths, strokes, and ED revisits within 1 year. Patient comorbidities, rhythms, and treatments were obtained by manual chart review. Patients were stratified into five groups based on ED presentation and management: electrical cardioversion, chemical cardioversion, spontaneous cardioversion, rate control, and no ED arrhythmia-specific treatment. Primary outcome was the combined 1-year rate of stroke or death; secondary outcomes included the proportion of eligible patients with conversion to normal sinus rhythm and the proportion of patients discharged home, stratified by treatment group. Results: A total of 753 consecutive patients with a primary ED diagnosis of atrial fibrillation were collected, with 25 deaths and 6 strokes $(4.1 \%, 95 \%$ CI $2.7-5.5 \%)$ in the following year. All deaths and strokes occurred in patients who were ineligible for rhythm control (31 of 300, 10.3\%). Patients undergoing electrical cardioversion achieved normal sinus rhythm $91 \%$ of the time, with $93 \%$ discharged home. Patients undergoing chemical rhythm control converted $41 \%$ of the time, with $93 \%$ discharged. Discharge rates of patients with spontaneous cardioversion, rate control, and no ED treatment were $96 \%, 73 \%$, and $85 \%$, respectively. Conclusions: In this two-centre cohort of ED patients with primary AF, the majority of patients were discharged home, but patients ineligible for rhythm control had a $>10 \%$ rate of stroke or death at 1 year. Keywords: atrial fibrillation, cardioversion, cohort study

\section{Syed, Shahbaz A}

asnsyed@ucalgary.ca

COMPUTER Physician Order Entry decreases time to medication delivery for high-acuity patients in the emergency department Shahbaz Syed, Eddy Lang, Dongmei Wang, Debbie Goulard, Thomas Rich, Grant Innes; Faculty of Medicine, University of Calgary, Calgary, $\mathrm{AB}$

Introduction: Computerized Physician Order Entry (CPOE) systems are designed to increase safety and improve quality of care, although impact on efficiency in the ED is less certain. This study examined the impact of CPOE on process times for medication delivery and laboratory utilization in the early, late, and control phases of a regional EDCPOE implementation. Methods: Setting: Three tertiary care hospitals serving a population in excess of 1 million inhabitants who initiated the same CPOE system during the same 3-week time window. Patients were stratified into three groupings: control, early CPOE, and late CPOE ( $n=100$ per group/hospital site). Eligible patients consisted of a stratified (40\% CTAS 2 and $60 \%$ CTAS 3 ) random sample of all patients seen 30 days preceding CPOE implementation (control), 30 days immediately after CPOE implementation (early CPOE), and 5-6 months after CPOE implementation (late CPOE). Primary outcomes were time taken (TT) from physician assignment (MD-sign) up to MDorder completion. The Student $t$-test was employed for statistical analysis. Results: In comparison with control, TT first MD-ordered medication decreased in both the early and late CPOE groups (102.6 minutes control, 62.8 early and 65.7 late, $p<.001$ ). TT first MDordered lab result increased in both the early and late CPOE groups compared to control $(76.4,85.3$, and 73.8 minutes, respectively, $p<$ $.001)$. TT first $\mathrm{x}$-ray also significantly increased in both the early and late CPOE groups $(80.4,84.8$ minutes, respectively, compared to 68.1 , $p<.001)$. There was no statistical difference found between TT discharge and consult request. Conclusions: Regional implementation of CPOE afforded important efficiencies in time to medication delivery for high-acuity ED patients. Increased times observed for laboratory and radiology results may reflect system issues outside the emergency department and as a result of potential confounding may not be a reflection of CPOE impact. Keywords: CPOE, emergency overcrowding, time to treatment

McLeod, Shelley L

shelley.mcleod@lhsc.on.ca

THE USE of anticoagulation prophylaxis in patients presenting to the emergency department with atrial fibrillation at high risk of stroke

Bongard AF, Richardson CA, McLeod SL; Division of Emergency Medicine, Faculty of Medicine, The University of Western Ontario, London, ON

Introduction: The emergency department (ED) provides the potential to identify untreated patients with atrial fibrillation (AF) at risk of ischemic stroke using the CHADS2 score. The objective of this study 
was to assess how well emergency physicians follow the current Canadian guidelines by identifying patients who are at high risk of stroke and initiating anticoagulation therapy in the ED. Methods: A retrospective medical record review was conducted for a random sample of adult ( $\geq 18$ years) patients with atrial fibrillation from the EDs of two academic tertiary care centres (combined annual census 140,000) between April 1, 2009, and March 31, 2010. Data were recorded by trained research personnel using a standardized data extraction tool. Results: Eighty-two patients with AF were included in this review. The mean (SD) age of patients was 69.8 (14.4) years, and 48 (58.5\%) were female. Thirty-nine (47.6\%) had a new diagnosis of AF in the ED Twenty-one $(25.6 \%)$ were already anticoagulated with warfarin, 21 $(25.6 \%)$ were already on aspirin, and $3(3.6 \%)$ patients were on both warfarin and aspirin. Of the $31(37.8 \%)$ patients with a CHADS2 score $\geq 2$ who were not previously on warfarin, 19 of $31(61.3 \%)$ were correctly identified and either started on warfarin $(n=2)$ in the ED or had follow-up arranged within 1 week $(n=17)$ to begin anticoagulation. Of the 37 patients who were not on warfarin or aspirin at the time of their ED visit, 7 (18.9\%) were started on aspirin in the ED. Conclusions: Our study demonstrates that emergency physicians are correctly identifying patients with $\mathrm{AF}$ at high risk of stroke and either starting them on warfarin in the ED or referring them within an appropriate time period to another physician for evaluation of anticoagulation. Keywords: anticoagulation, atrial fibrillation, CHADS2

\section{Colacone, Antoinette}

antoinette.colacone@mcgill.ca

STEPS toward alleviating boarding of psychiatric patients in the emergency department: evaluation of bed occupancy appropriateness on psychiatric wards

Marc Afilalo, MD, Jean-François Boivin, MD, ScD, Emmanuelle Jourdenais, MD, Stephen Rosenthal, MD, MSc., Xiaoqing Xue, MSc, Nathalie Soucy, PhD; Emergency Department, Jewish General Hospital, McGill University, Montreal, QC

Introduction: Bed accessibility for emergency department (ED) psychiatric patients is compromised when inpatients inappropriately occupy beds for extended durations. The study aims to identify characteristics and needs of psychiatric patients with prolonged hospital stay and explore alternative resources optimally meeting their needs. Methods: Psychiatric patients hospitalized for $30( \pm 3)$ days were enrolled in two teaching hospitals from March 2009 to April 2010. Data on sociodemographic, medical, psychiatric, and patient needs were collected via chart review and inquiries to medical/nursing staff. Appropriateness of hospitalization on day 30 was assessed using the Readiness for Discharge Questionnaire (RDQ) and expert consultations. Reasons responsible for inappropriate stay and adequate resources enabling discharge were identified. Results: A total of 261 psychiatric patients were enrolled. Mean age was $45 \pm 18$ years, and $97 \%$ were admitted from the ED. Prior to hospitalization, $56 \%$ were cognitively impaired, $46 \%$ had a legal representative, $36 \%$ had family support, $22 \%$ lived in a psychiatric group facility, and $10 \%$ were homeless During their stay, $67 \%$ had undergone social services assessment, $52 \%$ had occupational therapy, and $52 \%$ had a discharge plan initiated. Assessment with RDQ revealed that $22 \%(n=57)$ of hospitalizations at day 30 were deemed inappropriate due to discharge planning delays $(30 \%)$, legal issues $(25 \%)$, lack of alternative resources $(23 \%)$, counselling/education (7\%), and psychosocial issues (4\%) and required further investigation (4\%). Alternative psychiatric health care resources evaluated to better meet these patients' needs included LTC $(28 \%)$, outpatient ambulatory care (19\%), psychiatric subacute care $(19 \%)$, and home care (4\%). Conclusions: Inappropriate hospitalizations of psychiatric patients were mainly due to delays in discharge planning, ongoing legal issues, and lack of alternative resources. LTC, outpatient ambulatory care, and psychiatric subacute care were identified as more adequate resources for this population. Keywords: bed occupancy, psychiatry, readiness for discharge

Harris, Devin R

devinh@shaw.ca

SAFETY and efficiency of rapid assessment of transient ischemic attack (TIA) patients via an emergency department (ED) diagnosis and treatment unit (DTU)

Harris DR, Cheng L, Johnston DCC, Grafstein E, Stenstrom R, Scheuermeyer F; Department of Emergency Medicine, St. Paul's Hospital; Department of Emergency Medicine, University of British Columbia, Vancouver, BC

Introduction: The high risk of stroke after transient ischemic attack (TIA) can be reduced by $80 \%$ if patients are assessed and treated within 48 hours, but only $21.3 \%$ of patients in our province are seen by a neurologist within this time. For patients presenting to an emergency department (ED) with a TIA, an ED-based short-stay diagnostic and treatment unit (DTU) may provide an alternative to inpatient admission or outpatient investigations. Methods: Using literature-based best practices, the Departments of Emergency Medicine, Neurology, and Radiology collaborated to develop an expedited pathway to provide DTUbased investigations and secondary prevention for patients with TIA. To obtain outcomes, patients were probabilistically linked to the provincial stroke registry via a unique identifier. The primary outcome was stroke at 1 month; secondary outcomes were operational benchmarks including time to $\mathrm{MD}$, proportion of patients receiving advanced imaging (CT angiography and carotid ultrasound) and neurology consultation under 12 hours, proportion of patients admitted, and ED length of stay. Results: From Nov. 2009 to Aug. 2010, 22 patients were admitted to the DTU with a TIA; $59 \%$ were male; average age 67 years. No patient had a stroke within 1 month $(0 \%, 95 \%$ CI $0-2.3 \%)$. Median time to MD assessment was 10 minutes (IQR 6-56); $100 \%$ of patients underwent advanced imaging and neurology consultation $<12$ hours. ED LOS was 572 minutes (IQR 315-800). Four of $22(18.1 \%)$ were admitted. Conclusions: A DTU may provide a safe and efficient location for managing TIAs. Keywords: clinical decision unit, stroke, TIA

McLeod, Shelley L

shelley.mcleod@lhsc.on.ca

DO call volume and training level result in lower rates of paramedic error?

Paul Robinson, Matthew Davis, Adam Dukelow, Mike Lewell, Severo Rodriguez; Southwest Ontario Regional Base Hospital Program, London, ON

Introduction: The Southwest Ontario Regional Base Hospital Program provides medical direction, education, and quality assurance to 11 emergency medical services (EMS) systems with volumes ranging from 300 to 83,000 calls per year. The objective of this study was to compare call volume and level of paramedic training with the frequency of prehospital medical directive errors. Methods: All EMS calls over a 2-year period (April 2008-March 2010) were reviewed through a computerbased filtering process to identify any errors. Calls that involved a medically delegated act or were considered high risk (no service calls, field pronouncements) were manually audited by trained individuals. Errors were classified as critical (action or lack of action likely to produce mortality or increased morbidity, or performance of a controlled act that a paramedic is not authorized to perform), major (action or inaction that may cause morbidity, but not life or limb-threatening), or minor (action or inaction that likely did not affect patient morbidity). Errors that occurred in high-call-volume (HCV) services $(>25,000$ calls/year) were compared with those occurring in low-call-volume (LCV) services $(<$ 25,000 calls/year). Services were classified as those employing primary care paramedics (PCPs) and those employing both advanced care para- 
medics (ACPs) and PCPs. The error rates between the two types of services were compared. Results: A total of 35,559 calls were audited, and 1,419 errors were identified. Twenty-three (1.6\%) critical, 241 (17\%) major, and $1,155(81.4 \%)$ minor errors were made. The HCV services had an error rate of $2.1 \%$; the LCV services had an error rate of $6.5 \%(p<.01)$. Services that had a PCP level of care had a significantly higher rate of errors compared with those with a combined $\mathrm{ACP} / \mathrm{PCP}$ level $(4.6 \%$ v. $3.8 \% ; p<.01)$. Conclusion: Low-callvolume services have a significantly higher rate of medical directive errors. Services employing only PCPs have a significantly higher rate than those that employ both ACPs and PCPs. Keywords: call volume, EMS, medical directive errors

\section{Lang, Eddy S}

eddy.lang@albertahealthservices.ca

WHY did you go to the emergency department? Findings from the Health Quality Council of Alberta Urban and Regional Emergency Department Patient Experience Report

Lang E, Cooke T, Aguanno A, Cowell J, Rowe B, Macrae A, Zaver F; Department of Emergency Medicine, Foothills Medical Centre, University of Calgary, Calgary, $\mathrm{AB}$

Introduction: Understanding why patients seek ED care is relevant to policy development. This study describes patient motivations for seeking ED care in Alberta. Methods: Cross-sectional survey of a random patient sample from 12 urban/regional EDs in winter 2007 and 2009. A previously validated questionnaire, based on the British Healthcare Commission Survey, was distributed according to a modified Dillman protocol. Exclusion criteria: age $0-15$ years, left prior to being seen/treated, died during the ED visit, no contact information, presented with a "privacy"-sensitive case. Aggregate responses are reported unless statistically significant differences were identified. Sample weights were applied adjusting for interfacility sample proportion differences. Results: A total of 21,639 surveys were distributed. Response rate was $46 \%$. Patients sought ED care on the advice of a health care professional (35\%), after consulting with a friend/family member (34\%) and/or based on own assessment of need (34\%). The ED was perceived as "the best place for my problem" (46\% in 2007 and $48 \%$ in $2009 ; p=.03)$, "the only choice available at the time" (43\%), and/or "the most convenient place to go" (12\%). Most patients consulted the ED for a new illness $(32 \%)$ or new injury $(27 \%)$. The remainder of presentations were associated with pre-existing conditions: worsened chronic condition (23\%), complication of recent medical care $(13 \%)$, routine care $(2 \%)$, follow-up care $(2 \%)$, or other $(2 \%)$. Eighty-nine percent of patients had a "personal family doctor/specialist whom they saw for most of their health care needs," and 96\% of those patients had visited this physician within the past 12 months. Conclusions: Most patients consult with others prior to presenting for ED care. The decision to visit an ED is based on perceived need rather than convenience. The majority of presentations are related to new conditions, exacerbation of chronic conditions, or complications of prior care. Keywords: ED utilization, emergency overcrowding, survey

\section{Scheuermeyer, Frank}

frankscheuermeyer@yahoo.ca

\section{MANAGEMENT of young patients presenting to the emergency} department in atrial fibrillation

Heslop C, Scheuermeyer F; St. Paul's Hospital and the University of British Columbia, Vancouver, BC

Introduction: Young patients $(<50$ years of age $)$ presenting to an emergency department (ED) with atrial fibrillation have minimally investigated. We describe the management and 1-year outcomes of such patients. Methods: This retrospective cohort study of two urban EDs used an administrative database to identify consecutive patients with a primary discharge diagnosis of ECG-proven AF across a period of 30 months. Patient comorbidities, rhythms, and treatments were obtained by structured manual chart review. Patients were stratified based on attributed causes of AF: idiopathic, related to drug or alcohol misuse, or attributable to cardiac and systemic diseases. To ascertain long-term outcomes, patients were probabilistically linked to provincial vital statistics and stroke registries and the regional ED database. Primary outcome was the 1-year rate of stroke and death; the secondary outcome was the number of return visits to a regional ED in 1 year. Results: Ninety-five patients with 165 visits were reviewed; 59 had idiopathic AF, 13 with AF related to substance misuse, and 23 had cardiac or medical causes for their AF. Only two deaths were observed - both among patients in the latter category - and no strokes were reported in any group $(2.1 \%, 95 \%$ CI $0.0-5.0 \%)$. Return visit rates were highest for patients with idiopathic AF and lowest for patients with substance-related causes. Conclusions: Regardless of management strategy, young patients with idiopathic or substancerelated AF had excellent outcomes, while patients with medical comorbidities had the highest rate of mortality. Keywords: adverse outcomes, atrial fibrillation, cohort study

\section{Brison, Robert}

brisonr@kgh.kari.net

SPLINT, don't cast, your torus fractures

Robin Grant, Dr. Robert J. Brison

Queen's University School of Medicine, Kingston, ON

Introduction: Distal radius torus (buckle) fractures are common pediatric emergency department (ED) presentations. Conventional management involves circumferential forearm casts. Recent studies suggest that removable splints provide equal or better outcomes. Objectives of our study were to (1) assess for adverse outcomes with torus fractures and whether this varied by immobilization technique, (2) assess patterns of practice related to immobilization of these fractures in one academic centre, and (3) assess for variation in resource utilization by immobilization technique. Methods: A 5-year (2005-2009) retrospective medical record review of torus fractures in children $\leq 10$ years of age was conducted in the two academic EDs in Kingston, Ontario. Forearm fractures were identified using injury surveillance data from two ED-based sources. From these sources, ED and radiology records were used to identify torus fractures. Patients were followed using medical records for 6 months to assess for malunion, malalignment, or recurrent fracture; choice of initial and subsequent immobilization technique; and numbers of and reasons for return visits to ED or fracture clinic. Results: We identified 154 torus fractures. There were no adverse outcomes at 6 months postinjury, irrespective of immobilization technique. ED management involved circumferential casting for $115(74.7 \%)$, splinting for $36(23.4 \%)$, and no immobilization for 2 . The median number of follow-up visits for children casted and splinted was two; however, $58.6 \%$ of return visits for children splinted were for replacement of their splint with a cast. Eleven casted and five splinted had return visits for device repair. Conclusions: Children with torus fractures do not have adverse outcomes. There is a greater requirement for ED and clinic-based follow-up for patients who are casted versus those splinted. Our study supports literature suggesting a splint-only strategy for torus fractures of the distal radius. Keywords: health records review, splint-only strategy, torus fractures

McLeod, Shelley L

shelley.mcleod@1hsc.on.ca

PREDICTORS of mortality in the septic emergency department patient: a 5-year review

Arbeau RP, McLeod SL, Doran S, Sedran RJ; Division of Emergency Medicine, Faculty of Medicine, The University of Western Ontario, London, $\mathrm{ON}$ 
Introduction: Hypotension and elevated lactate in the setting of an infection are used to diagnose the septic patient in the emergency department (ED). However, various physiologic parameters, demographic characteristics, and clinical laboratory findings may help identify those ED patients with severe sepsis who are at increased risk of mortality. The objective of this study was to identify predictors independently associated with mortality in ED patients with sepsis. Methods: A retrospective medical record review was conducted for all adult ( $\geq 18$ years) patients admitted from the ED of an academic tertiary care centre (annual census 75,000) with a primary diagnosis of sepsis from 2005 to 2009. Data were extracted using a standardized data collection tool by trained research personnel. Gender, age, triage score, triage vitals (heart rate, respiratory rate, blood pressure, and oxygen saturation), antibiotic use, resuscitative fluid use, lactate level, white blood cell count, corticosteroid use, vasopressor use, need for intubation, and mortality were recorded. Backwards stepwise multivariable logistic regression models determined predictor variables independently associated with mortality. Results: A total of 348 patients were identified; 28 were excluded as direct admissions and 7 charts were unavailable, leaving 313 included in the review. The mean (SD) age was 64.8 (18.8) years, and $53.7 \%$ were male. Sixty of $313(19.2 \%)$ patients did not survive to hospital discharge. In the multivariable model, SBP $\leq 90$ $\mathrm{mm} \mathrm{Hg}$ (OR 4.5; 95\% CI 2.8, 6.1), RR $\geq 20$ (OR 4.0; 95\% CI 2.7, 5.3), lactate $\geq 4.0$ (OR 3.4; 95\% CI 2.6, 4.2), and age $\geq 65$ (OR 1.9; 95\% CI $1.2,2.6)$ were identified as significant predictors of mortality. Conclusion: Predictors of mortality in septic ED patients were physical or physiologic parameters, which are easily obtainable to alert the clinician to the patient who requires more immediate and aggressive medical attention. Interestingly, medical interventions did not demonstrate significant prognostic value. Keywords: health records review, mortality prediction, sepsis

McLeod, Shelley L

shelley.mcleod@lhsc.on.ca

ANALGESIA in the emergency department management of suspected renal colic

Yan JW, McLeod SL, Edmonds ML, Theakston KD, Sedran RJ; Division of Emergency Medicine, Faculty of Medicine, The University of Western Ontario, London, ON

Introduction: Pain from renal colic can be debilitating and often requires repeated administration of multiple analgesics to obtain adequate pain control in the emergency department (ED). Current practice patterns for pain control in the ED vary widely and may involve narcotics, nonsteroidal antiinflammatories (NSAIDs), or both. Furthermore, it is unclear as to the type of analgesia physicians are prescribing for pain postdischarge. The objective of this study was to determine the use of analgesia in patients with suspected renal colic in the ED and the prescribing patterns of physicians for outpatient management of recurrent renal colic pain. Methods: This was a prospective observational cohort study involving all adult ( $\geq 18$ years) patients who presented to the EDs of a tertiary care centre (combined census 150,000 ) with suspected renal colic over a 3-month period (Oct-Dec 2010). Trained study personnel reviewed the charts to determine if analgesics were administered, the type of analgesic given, time from triage to first administration of analgesia, and if the patient was prescribed analgesics on ED discharge. Results: Of 127 patients enrolled, 92 (72.4\%) received analgesia in the ED. Of those receiving analgesia, $34(37.0 \%)$ received NSAIDs alone, $8(8.7 \%)$ received narcotics alone, and 48 $(52.2 \%)$ received both NSAIDs and narcotics. Two $(2.2 \%)$ patients received Tylenol alone. The median (IQR) time from triage to analgesia was $74.5(48.5,125.5)$ minutes. Seventy-four $(58.3 \%)$ patients were given prescription analgesics on discharge. Two of these patients $(2.7 \%)$ received NSAIDs alone, $6(8.1 \%)$ received NSAIDs and narcotics, and $66(89.2 \%)$ received narcotics alone. Conclusions: Analge- sia use in the ED for suspected renal colic remains variable, although most patients receive NSAIDS with or without narcotics. The majority of patients who receive prescriptions for outpatient management of pain are given narcotics. The prolonged time to analgesia suggests that improvements can be made in the treatment of acute renal colic pain. Keywords: analgesia, health records review, renal colic

\section{Campbell, Samuel G}

emsgc@cdha.nshealth.ca

THE UTILITY of end-tidal capnometry during procedural sedation and analgesia in the emergency department: does it make a difference?

Campbell SG, Magee KD, Zed PJ, Froese P, Etsell G, LaPierre A, MacKinley RP, Butler MB; Dalhousie University, Halifax, NS

Introduction: Medications utilized during PSA in the emergency department (ED) have the potential for respiratory and hemodynamic adverse events. Traditionally, respiratory monitoring during PSA uses pulse oximetry; however, end-tidal capnometry $\left(\mathrm{ETCO}_{2}\right)$ has been advocated to improve patient safety during PSA. We sought to evaluate the impact of $\mathrm{ETCO}_{2}$ monitoring compared to standard pulse oximetry in patients undergoing ED paramedic-administered PSA. Methods: This was a prospective, randomized, unblinded trial on consenting patients undergoing ED PSA at the Halifax Infirmary. Patients were randomized to monitoring with or without $\mathrm{ETCO}_{2}$ in addition to the current standard of care. Primary end points included respiratory adverse events, with secondary end points of level of sedation, hypotension, other PSA-related adverse events, and patient satisfaction. Results: A total of 986 patients were randomized, 501 to usual care and 485 to additional $\mathrm{ETCO}_{2}$, of whom $48 \%$ were female with a mean (SD) age of 46 (18) years. Orthopedic manipulations (71\%), cardioversion (12\%), and abscess incision and drainage $(12 \%)$ were the most common procedures, and propofol/fentanyl was the sedative/analgesic combination used for most patients. There was no difference between the two groups in patients experiencing desaturation $<90 \%$; however, patients in the $\mathrm{ETCO}_{2}$ group were more likely to require airway repositioning $(12.9 \%$ v. $9.3 \%, p=.003)$. There was no difference in the need for airway intervention. Time taken for the procedure, vomiting, degree of "overshoot," and patient satisfaction were not different between groups. Hypotension (SBP $<100 \mathrm{~mm} \mathrm{Hg}$ or $<85 \mathrm{~mm}$ $\mathrm{Hg}$ if baseline $<100 \mathrm{~mm} \mathrm{Hg}$ ) was observed in $6(3.3 \%)$ patients in the $\mathrm{ETCO}_{2}$ and $7(1.4 \%)(p=.048)$. Conclusions: The addition of $\mathrm{ETCO}_{2}$ does not appear to change clinically significant outcomes. It is, however, associated with an increase in both the use of airway repositioning maneuvers and hypotension. We do not believe that $\mathrm{ETCO}_{2}$ should be recommended as standard for the monitoring of patients undergoing PSA. Keywords: end-tidal capnography, procedural sedation and analgesia, randomized controlled trial

McLeod, Shelley L

shelley.mcleod@1hsc.on.ca

DO radiation exposure risks influence diagnostic imaging choices for investigating pulmonary embolism in an adult emergency department?

Ahn JS, Edmonds ML, McLeod SL, Dreyer JF; Division of Emergency Medicine, Faculty of Medicine, The University of Western Ontario, London, ON

Introduction: Current literature suggests that radiation from diagnostic imaging increases the lifetime risk of cancer in patients, especially for younger patients and females. However, there are no guidelines that limit the amount of radiation exposure to patients, nor is informed consent required for diagnostic imaging. The investigation of pulmonary embolism (PE) is of particular interest because both the ventilation-perfusion (VQ) scan and CT pulmonary angiography 
(CTPA) can effectively diagnose or exclude PE. However, the dose of ionizing radiation is at least five times greater with CTPA. The objective of this study was to determine which investigations emergency physicians would order for specified patient populations, especially those in high-risk groups. Methods: An online survey was sent to all 42 adult emergency physicians working at the London Health Sciences Centre using Survey Monkey to establish imaging choices for investigating PE and determine physicians' baseline knowledge of the radiation risks associated with these tests. Results: Thirty-one (74\%) physicians completed the survey. The majority of physicians (76-84\%) chose VQ as the preferred modality for investigating PE in patients $<45$ years old. The number of CTPAs ordered increased for patients $>60$ years old and those with comorbidities such as COPD. Only a minority of respondents knew the correct radiation dose of a VQ scan (37.5\%) and CTPA (32\%). Conclusions: This study revealed that despite limited knowledge of radiation exposures, the majority of physicians surveyed preferentially chose the study with the lower dose of ionizing radiation for younger patients. Survey results will be compared to a retrospective chart review. Keywords: CT imaging, pulmonary embolism, radiation

\section{Varner, Catherine E}

catherine.varner@gmail.com

PAIN protocol decreases time to analgesia in adult patients with renal colic

Catherine Varner, Stella Yiu, Tamara Arenovitch, Steven Friedman, Megan Reynolds, Caesar Lim, Paul Ellis, David Carr; Department of Emergency Medicine, University Health Network, Toronto, ON

Introduction: Time to analgesia is a recognized quality indicator for assessing emergency department (ED) care. Triage-driven protocols in patients presenting to the ED with symptoms of renal colic (RC) have demonstrated improved time to analgesia and patient satisfaction but have not measured the impact on ED length of stay (ED LOS). We sought to assess impact of an analgesia protocol for suspected RC on ED LOS and time to analgesia. Methods: This study was conducted in the two ED sites of an urban teaching hospital over a 12-month period. A retrospective chart review of consecutive patients with an ED diagnosis of renal colic explored use of the RC protocol, patient demographics, triage vital signs, time to analgesia, and ED LOS. Exclusion criteria included patients with unstable vital signs at presentation, age $<18$ years, and repeat visits in the study period. Results: The protocol was initiated in $80(14 \%)$ of 574 patients meeting inclusion criteria and was more likely to be used in patients reporting a prior history of RC (OR 3.64; 95\% CI 1.63-8.16; $p=.0002$ ) and higher pain scores (OR 1.52; $95 \%$ CI $1.15-2.02 ; p<.0001)$. Protocol use was associated with reduced length of time between registration and first analgesic $(82.0$ minutes v. 44.0 minutes, $p<.0001$ ) but was not associated with reduced length of stay (304.5 minutes v. 306.0 minutes, $p=.5260$ ). Conclusions: A protocol to expedite pain management in renal colic patients was associated with decreased time to analgesic but not decreased ED LOS. However, the protocol was only used in a minority of patients, suggesting that potential barriers to wider use of the protocol should be studied. Keywords: analgesia, pain protocol, renal colic

Yeung, Marianne Y.T.

myeung@ottawahospital.on.ca

EMERGENCY department management of nontraumatic pneumothorax using a small-bore "pigtail" chest catheter

Marianne Yeung, W. Joseph Kozar, Sudhir Sundaresan, A. Adam Cwinn, Ian G. Stiell; Department of Emergency Medicine, University of Ottawa, Ottawa, ON

Introduction: We developed an outpatient protocol whereby low-risk primary spontaneous or iatrogenic pneumothorax patients are treated by ED physicians using a small-bore pigtail catheter and then discharged for outpatient follow-up. Our goal was to evaluate the effectiveness and safety of this protocol. Methods: We conducted a prospective cohort study at two large academic EDs and included a convenience sample of adults with nontraumatic pneumothorax. Excluded were patients with age $<17$ or $>40$, abnormal vital signs, $>$ 25 -pack-year smoking history, INR $>1.1$, pleural fluid, tension pneumothorax, or coincident second illness. Thirty-four ED staff and 28 resident physicians were trained using instructional video, simulation task trainers, and printed and online documents. The protocol has the ED physician insert a pigtail catheter and connect it first to an underwater seal and later to a one-way valve. The stable patient with a fully reexpanded lung is discharged home for next-day assessment at the thoracic surgery clinic, with catheter removal several days later. We prospectively assessed ED physician comfort with the protocol, admissions, and complications. Results: Forty-seven eligible patients were mean age 25.7 , male $76.6 \%$, first time $44.7 \%$, spontaneous $59.6 \%$, large pneumothorax $63.8 \%$, admitted $36.2 \%$. Thirty-one chest catheters were inserted by 30 physicians. ED physicians indicated comfort with the protocol in $95.7 \%$ of cases and easy catheter insertion in $95.0 \%$ of cases. Of pigtail cases, thoracics was consulted in $32.2 \%$, usually due to residual pneumothorax; $77.4 \%$ were discharged from the ED to be managed as outpatients; $12.9 \%$ were admitted later. $93.6 \%$ of patients had good outcomes; there were no deaths, 1 reexpansion pulmonary edema, 1 catheter that required reinsertion, and 2 unscheduled visits for minor issues. Conclusion: ED physicians effectively and safely implemented a pigtail catheter insertion protocol for pneumothorax patients, and this approach has been adopted permanently at the participating EDs. Keywords: cohort study, pigtail catheter, pneumothorax

\section{Friedman, Steven M}

aclsdoc@hotmail.com

INCIDENTAL findings of elevated random plasma glucose in the ED as a prompt for outpatient diabetes screening

Brenda Baswick, BA, MD, Andrea Scott, BSc, MSc, MB, BCh, Janaki

Vallipuram, BSc (Hon), Steven Marc Friedman, MD, MPH

University Health Network, University of Toronto, Toronto, ON

Introduction: Approximately 3 to $5 \%$ of Canadians are thought to have undiagnosed type 2 diabetes, and many do not have routine primary care. Random plasma glucose (RPG) has been shown to be correlated with glycosylated hemoglobin and proposed as a screening tool. The aim of the study is to determine if incidental findings of elevated RPG in emergency department (ED) patients without known diabetes/prediabetes is a useful screen for impaired glucose metabolism. Methods: A retrospective cohort study was conducted in an urban teaching hospital using chart review of all ED patients over a 1month period. Patients with RPG $>7.0 \mathrm{mmol} / \mathrm{L}$ were recruited for screening and follow-up interviews and encouraged to attend their family physician (FP) for confirmatory testing. Exclusion criteria included age $<18$, known diabetes, admission to hospital, and inability to consent. Results: Approximately one-third (33.1\%, $n=1,149$ of $3,470)$ of ED patients had RPG drawn. RPG $>7.0 \mathrm{mmol} / \mathrm{L}$ was detected in $24.2 \%(n=278)$. After excluding $41.0 \%(n=114$ of 278$)$ with diabetes and $10.4 \%(n=29)$ for criteria including age, admission status, and inability to consent, 143 met inclusion criteria. Unreachable or deceased patients numbered $14.0 \%(n=39)$, and $1.1 \%(n=3)$ declined participation. Telephone interviews were completed for $61.5 \%$ ( $n=88$ of 143$)$ meeting inclusion criteria, and they were advised to seek confirmatory testing; $72.7 \%(n=64$ of 88$)$ followed up with their FP; $11.4 \%(n=10)$ of patients had abnormal fasting plasma glucose and were prescribed lifestyle and diet modifications; and $1.1 \%(n=1)$ were started on an oral hypoglycemic agent. Conclusion: The incidental finding of an elevated RPG in ED patients may be a useful screening tool to prompt for further investigation for 
undiagnosed prediabetes and diabetes. Keywords: prediabetes, random plasma glucose, screening

Sibley, Aaron K

asibley@ualberta.ca

THE EFFECT of an inner-city emergency medicine rotation on medical students' and first-year residents' attitudes toward the homeless

Sibley AK, Dong KA, Rowe BH; University of Alberta, Edmonton, $\mathrm{AB}$

Introduction: Homeless persons are frequent users of the emergency department (ED) and health care in general. Medical teaching about homelessness is limited and dispersed throughout the curriculum; changing medical school demographics may mean fewer students have been exposed to this population. We sought to determine if an innercity emergency medicine (EM) rotation changes the attitudes of medical students and first-year residents toward the homeless population. Methods: This prospective cohort study included first-year residents as well as third- and fourth-year medical students completing a rotation in EM at the Royal Alexandra Hospital in Edmonton, Alberta. Participants were asked to complete an electronic questionnaire both pre- and postrotation. Data collected included demographics plus scores on The Health Professionals' Attitudes Towards the Homeless Inventory (HPATHI). The HPATHI is a 19-item questionnaire designed for health care providers and validated elsewhere. Results: Sixty-six learners (medical student 49 [71\%], resident 19 [29\%]) completed either the prerotation $(n=18)$, postrotation $(n=8)$, or both $(n=40)$ questionnaires (response rate 67\%). Most participants were male (42 [64\%]), single (47 [71\%]), and $\leq 30$ years of age (59 [89\%]). The majority of participants were of a Caucasian or East/South Asian background (61 92\%]) and grew up in an urban setting (51 [77\%]). Fortysix $(96 \%)$ participants saw at least one homeless person during their rotation. A Wilcoxon test was performed to compare HPATHI scores pre- and postrotation. There was no significant difference between pre and post aggregate scores $(z=-0.78, p=.44)$ or any of its three subscales (Personal Advocacy, Social Advocacy, and Cynicism). Conclusions: First-year residents and medical students are frequently exposed to homeless patients during an inner-city EM rotation. Attitudes toward the homeless did not significantly change following the rotation. Educational objectives should be set to improve attitudes of learners toward the homeless throughout their rotation. Keywords: homelessness, medical education, survey

Rowe, Brian $\mathrm{H}$

brian.rowe@ualberta.ca

DIFFERENCES in outcome for adult patients presenting with hypoglycemia: a Canadian multicentre study

Rowe BH, Singh M, Vila-Roel C, Edmonds M, Lang E, Sivilotti M, Scheuermeyer F, Worster A, Riley J, Afilalo M, Stiell I, Campbell $\mathrm{S}$, for the CAEP Research Consortium; Department of Emergency Medicine, University of Alberta, Edmonton, AB, and CAEP RC, Ottawa, ON

Background: Adverse events from hypoglycemia result in presentations to the emergency department (ED), which can be complex and difficult to treat. Objectives: This study was designed to examine outcomes for patients with type 1 and type 2 diabetes mellitus (DM) presenting to EDs with hypoglycemia. Methods: A retrospective chart review was conducted at 10 adult EDs across Canada. ICD-10 codes were used to identify applicable cases at all sites, and randomized samples were obtained. A structured chart review was completed on patients with a documented blood sugar of $<3.9$ and DM by trained research nurses using standard chart review methodology. Results are presented as proportions and medians with interquartile range (IQR).
The main outcome of interest was treatments in the ED and outcomes. Results: A total of 1,025 patients $>17$ years old were included in the study; 387 (38\%) were type 1 and $638(62 \%)$ were type $2 \mathrm{DM}$. Males predominated in type 2 ; type $2 \mathrm{DM}$ were older ( 72 v. $53 \%$; $p=.001)$. Most patients arrived by ambulance, and triage scores revealed severe presentations in $39 \%$ of cases. There were no differences in hypoglycemic presentations associated with injuries $(9.6 \%$ v. $9.2 \%)$. Overall, patients with type $2 \mathrm{DM}$ were admitted to the hospital more frequently than type 1 ( $31 \%$ v. $10 \% ; p=.012)$; few patients with DM left against medical advice ( $3.6 \%$ v. $1.9 \% ; p=.08)$. No hospitalizations in any Canadian site were to an endocrinology service. Discharged instructions were documented more often in type 1 than type $2 \mathrm{DM}$ hypoglycemia cases $(62.5 \%$ v. $50 \% ; p<.001)$. Conclusion: Patients presenting with hypoglycemic episodes due to type $2 \mathrm{DM}$ are often older, have more comorbidities, and require hospitalization more often. Admission to generalist physicians in Canada differs from other locales, and discharge instructions vary considerably. These issues should be considered in continuing medical education activities. Keywords: diabetes, health records review, hypoglycemia

Han, Vanessa

vanessa.han@utoronto.ca

RISK factors for youth homicide

Vanessa Han, Dan Cass, Jim Edwards, Carolyn Snider

University of Toronto, Toronto, ON

Introduction: Homicide is the fourth leading cause of death among Canadian youth aged 15 to 24 . As an increasing number of youth present to emergency departments (ED) with violent, intentional injury, there is an absence of guidelines and protocol regarding safe discharge and secondary prevention. This is perhaps due to a lack of screening tools to identify those who are at highest risk for repeat injury and death. The aim of this study was to determine the prevalence of demographic, social, and behavioural characteristics among youth victims of homicide. Methods: Data were extracted from cases of youth homicide investigated by the Office of the Chief Coroner of Ontario in the years 2006 and 2007. Age, sex, cause of death, environment of death, personal background, and social and behavioural factors, such as alcohol and drug use, were collected and analyzed. Results: A total of 106 cases were reviewed. Gunshot wound was the most common cause of death $(44.3 \%$; 95\% CI 35.3-53.8\%). The homicides most often occurred in an urban, outdoor setting (44.3\% CI 35.3-53.8\%), and $65.1 \%$ of victims had positive toxicology for recreational drugs (CI $55.6-73.5 \%$ ) and $44.3 \%$ for alcohol (CI $35.3-53.8 \%$ ); $28.3 \%$ of the study population had a documented criminal record (CI 20.6-37.5\%); $89.6 \%$ of victims were male (CI 82.4-94.1\%); $14.3 \%$ were of First Nations ethnicity (CI 7.1-26.7\%); and 37.9\% were born outside Canada (CI 26.6-50.8\%), notably elevated compared to 2006 census data on the population of Ontario aged 15 to 24. Conclusions: Victims of youth homicide are disproportionately of the male gender, of First Nations ethnicity, and born outside Canada. The majority exhibit evidence of drug or alcohol use, and a significant proportion has engaged in prior criminal behaviour. These identified characteristics may be potential risk factors for youth homicide. Future studies will seek to quantify the predictive value of these risk factors and potential for incorporation into ED screening tools for risk of repeat violent injury and death. Keywords: epidemiology, homicide, screening

Patocka, Catherine

catherine.patocka@mail.mcgill.ca

EVALUATION of an emergency department triage screening tool for severe sepsis

Catherine Patocka, MD, Joel Turner, MD, FRCP, Xiaoqing Xue, Eli Segal, MD, FRCP; McGill Emergency Medicine Residency Program, Royal Victoria Hospital, Montreal, QC 
Introduction: Early goal-directed therapy has been shown to decrease mortality; however, identification of septic patients can be challenging. To improve detection of septic patients presenting to the emergency department (ED), we implemented a triage screening tool. Our study sought to determine the effect of this tool on time to antibiotic. Methods: This pre-post study was conducted in the ED of a tertiary teaching hospital in Montreal. Patients in both cohorts were identified by review of admission diagnosis and diagnosis on ED death certificate for a 1year period before and after introduction of the triage tool. Patients who met the criteria for severe sepsis or septic shock were identified and their charts reviewed to determine the length of time between triage and antibiotic administration. Multiple linear regression analysis was conducted to evaluate the effect of the triage tool while controlling for the effect of level of triage acuity based on the Canadian Triage and Acuity Scale (CTAS). Results are presented using time delays in minutes with its $95 \%$ confidence interval $(95 \% \mathrm{CI})$. Results: We identified 185 patients with severe sepsis or septic shock in the pretriage tool implementation group and 170 patients in the post-triage tool implementation group. The mean time to antibiotics (+SD) in the pre and post cohorts was $283(+213)$ minutes and $207(+150)$ minutes, respectively. Statistical analysis revealed that time to antibiotics decreased 69 minutes (95\% CI 34-106, $p=.0003$ ) overall post-triage tool implementation. For patients with CTAS levels $1-2$, the time to antibiotics decreased 101 minutes $(95 \%$ CI $63-139, p<.0001)$ versus patients with CTAS 3-5. Conclusions: The use of a sepsis triage screening tool significantly decreased the mean time to antibiotics in patients presenting to the ED who met criteria for severe sepsis or septic shock. Keywords: screening, sepsis, time to antibiotics

\section{Ackery, Alun}

\section{alun.ackery@utoronto.ca}

HOCKEY violence and injury in Canada: the experience of referees Ackery A; Tator CH; Snider C; Division of Emergency Medicine, Department of Medicine, University of Toronto, Toronto, ON

Introduction: Canadian emergency physicians commonly see concussion and other injuries from hockey. To date, there is very little understanding of the role of violence in hockey. Referees are the enforcers of on-ice rules and are a major factor in player conduct and safety. They are also subject to both verbal and physical abuse from players, coaches, and fans. We surveyed hockey referees in Canada from various leagues and from all levels of play. The purpose of this study was to answer the following question: What are the perceptions and roles of referees about violence and injury in hockey games? Methods: Using an online survey program (Survey Monkey) and Dillman methodology, we contacted referees across Canada. We gathered demographic information and posed questions (using predominantly Likert scales) on aggression and experience in hockey games. All information was collected anonymously. Results: The majority of referees $(N=632)$ indicated that violence is a serious concern to both players and referees at all levels of hockey. More than $90 \%$ of referees responded that they were the recipients of aggression and anger $(92.1 \%, 95 \%$ CI $90.0-94.2), 55 \%$ had been involved in hockey games where aggressive behaviour resulted in the referee losing control of the game $(55.2 \%, 95 \%$ CI $51.2-59.2)$, and $71 \%$ said that this increased aggression leads to injury $(71.0 \%, 95 \%$ CI $67.4-74.5)$. Referees' opinions are that coaches best manage on-ice safety (63\%). More experienced referees, compared to less experienced referees, perceive that concussion rates are increasing due to aggressive play $\left(\chi^{2}=51.81, p\right.$ $<.001)$. Conclusions: Hockey is an aggressive sport. Referees are important for hockey safety and need to be appropriately supported. Our paper studies referees and their experience with violence and hockey safety. Increased aggression can lead to injury. Referees believe that rules need to be enforced more diligently and that increased education is needed to guide parents, coaches, and players to make hockey safer. Keywords: head trauma, hockey, online survey
Rowe, Brian $\mathrm{H}$

brian.rowe@ualberta.ca

EXPLORING common reasons for consultation delays in tertiary care emergency departments

Brick C, Kokotilo A, Loewen B, Lowes J, Lovstrom L, Couperthwaite S, Dong K, Lang E, Villa-Roel C, Rowe BH; Department of Emergency Medicine and School of Public Health, University of Alberta, Edmonton, AB; Division of Emergency Medicine, University of Calgary, Calgary, $\mathrm{AB}$

Introduction: Consultations for specialty services are an important aspect of patient flow in emergency departments (ED). Delays in consultation contribute to ED overcrowding; this project was designed to examine the delays associated with consultations in greater detail. Methods: Prospective cohort study of convenience shifts from volunteering physicians at university (U) and universityaffiliated (U-A) high-volume EDs. Following informed consent, physicians completed standardized consult forms on all patients in whom a consultation was requested. Administrative data were used to record triage level and times of triage, placement, MD assessment, and consult request. Physicians commented on consult delays and completed a "reason for delay" based on their knowledge of the clinical environment at the time of consultation. Decision times (consult to bed request for admitted and ED discharge) were extracted independently by two reviewers; length of stay (LOS) results are presented. Continuous data were compared using Mann-Whitney tests, and proportions were compared using chi-square statistics. Results: Overall, 841 patient consults were documented; $38 \%$ were seen by a learner first in both sites. The median number of consults/patient was 1 (IQR 1, 1); however, 93 (11\%) involved more than one consultation. Although general internists were most commonly consulted, via residents/interns, the U-A proportion was higher $(22 \% \mathrm{v} .13 .6 \%$; $p=$ $.03)$. Overall, $52 \%$ of ED physicians consulted for admission and $61 \%$ were eventually admitted. Perceived consultation delays were recorded in $20.4 \%$ of cases. The most common reasons for delay were busy service $(29.6 \%)$, problems reaching the resident $(10.5 \%)$, and delayed consultant arrival $(9.3 \%)$. Conclusions: Perceived delays occur in over $20 \%$ of ED consultations resulting from overextended consult services. Efforts to reduce consultation decision-making time ED will require balancing consultant volumes with physician staffing. Keywords: emergency crowding, specialty consultations, throughput delays

\section{Bryan, Jennifer}

jennifer.bryan@utoronto.ca

A SYSTEMATIC review of serum lactate and sepsis outcome

Bryan JM, Freeman K, and Ellis P; Division of Emergency Medicine, University of Toronto, Toronto, ON

Introduction: Serum lactate has been used as a tool for prognostication in sepsis; however, the utility of serum lactate measurement is controversial. Further, it is unclear whether there is a critical serum lactate value. The objectives of this study are to (1) determine whether lactate level is associated with sepsis outcome and (2) determine whether there is a lactate cutoff level above which poor outcome in sepsis is more likely. Methods: This is a systematic review. PubMed and all Cochrane Library databases were searched for relevant articles. Included studies were limited to those reporting lactate level and mortality in patients with sepsis. Studies were identified by using MeSH terms, select keywords, and reference mining of existing reviews. Only studies published in English that involved adult patients were included. There were no publication date limits. Observational studies, randomized controlled trials, and systematic reviews were all eligible for inclusion. Editorials, clinical overviews, commentaries, practice guidelines, and case reports were excluded. Abstracts (and complete articles 
where indicated) were then assessed to ensure that studies met inclusion/exclusion criteria. The QADAS tool was used to assess study quality. Results: Eighty-four articles were identified, of which, on abstract review, 20 studies were found to meet inclusion/exclusion criteria. Due to the variability in nature of these studies, criteria were not met for pooled analyses/meta-analysis; analyses will therefore focus on summary data. Conclusions: This review will summarize the current state of our understanding of whether lactate is a useful measure in sepsis as well as whether there is a lactate level that could be considered a cutoff in predicting which patients are at most risk of poor outcome. Additional study in this area is clearly indicated, as is additional assessment of whether demographic variables (such as age and sex) act to mediate/moderate any relationship between serum lactate and outcome. Keywords: sepsis, serum lactate, systematic review

\section{Leaver, Chad A}

chad.leaver@ices.on.ca

DETERMINANTS of implementation success in a system-wide intervention to improve hospital performance and reduce emergency department waiting times

Schull MJ, Leaver CA, Vermeulen M, Salkeld E, Amit Nigam, Anne Sales, Debra Carew, Brian Rowe, Brian Golden, Astrid Guttmann, Robert Bell, Marc Afilalo, Therese Stukel, Merrick Zwarenstein; Institute for Clinical Evaluative Sciences, Toronto, ON

Introduction: Ontario's Ministry of Health is undertaking an emergency department process improvement program (ED PIP) aimed at reducing ED length of stay (ED LOS), improving in-patient flow, and enhancing quality improvement skills at 90 acute care hospitals. Methods: We administered an exit survey at the end of the 7-month ED PIP intervention to leadership teams from 21 hospitals that had completed the program by April 2010. The survey examined change process factors (goal setting, skill development, feedback, management control) and perceived implementation success (positive impact on patient flow, ED LOS, and behaviour change). Descriptive and linear regression analyses were used to evaluate the effect of change processes on perceived implementation success. Hospital volume and other contextual factors were included in the model. We used GEE methods to account for clustering of outcomes within EDs. Results: A total of 221 staff from leadership teams completed the survey: senior admin (31.6\%); ED physicians (4.1\%), ED nurses $(22.5 \%)$, general internal medicine (GIM) physicians $(0.4 \%)$, GIM nurses (12.4\%), allied health (7.8\%), and corporate/admin staff (21\%). Perceived implementation success varied across sites and professional groups. Factors associated with perceived implementation success for both waves were management control (the presence of effective leadership support) ( $\beta$ 0.18, 95\% CI 0.072, 0.29) and a context factor: extent to which process improvement initiatives had been implemented $(\beta 0.18$, $95 \%$ CI $0.084,0.27$ ). An organizational culture that is open to trying new things, having data feedback mechanisms, and the degree to which changes were believed to impact the organization broadly were also significant. Conclusions: Improving hospital performance is complex and requires engaged and supportive senior leadership. Results suggest that piloting and then fully implementing successful improvement initiatives may be more important to the perceived success of the program than other factors, such as goal setting and skill development. Keywords: emergency overcrowding, implementation, system-wide intervention

Chan, Teresa M.

teresa.chan@medportal.ca

UNDERSTANDING communication between emergency and consulting physicians: what are the differences between clinical specialties during the referral-consultation process?

Teresa Chan, BEd, MD; Donika Orlich, BSc; Kulamakan Kulasegaram, HBSc; Jonathan Sherbino, MD, MEd; Division of Emergency Medicine, McMaster University, Hamilton, ON
Introduction: Consultations and referrals are a very common occurrence in the emergency department. Our objective was to identify differences between emergency medicine (EM), internal medicine (IM), and general surgery (GS) physicians during the referral-consultation process. Methods: From March to September 2010, 61 physicians (31 residents and 30 attendings; 21 EM, 20 GS, 20 IM) were interviewed by a single interviewer. Two investigators independently reviewed $100 \%$ of the transcripts using grounded theory to generate a code of categories until saturation was reached. Coding disagreements were resolved by consensus, yielding a single inventory of themes, subthemes, and qualifiers. All of the transcripts were coded using the common code. Thirty percent of the transcripts were coded by a second investigator to determine interrater agreement. Results: A total of 245 themes and qualifiers were identified. Interrater agreement was $77 \%$. EM and IM physicians valued summarizing investigations and management more than GS (EM and IM 100\% v. GS 70\%). Descriptions of history and physical exam were more often endorsed by GS and IM (both $100 \%$ ) than EM $(81 \%)$. The reason for referral was deemed highly important by EM (95\%), but both IM and GS thought it less important (GS 55\%, IM 60\%). There were overall differences between the most highly endorsed elements $(>60 \%$ endorsement) in each group. GS and IM groups had very different top endorsement lists ( $p=$ .002 ). EM physicians had an endorsement list that did not vary from the other groups significantly (EM v. IM $p=.46$; EM v. GS $p=.08$ ). Conclusions: To our knowledge, this is the first study to identify the differences in the referral-consultation process between emergency medicine, internal medicine, and general surgery physicians. The EM group's top endorsed items were not significantly different from that of either IM or GS (in isolation). These findings may inform the development of referral-consultation model for junior learners. Keywords: communication, qualitative research, specialty consultations

\section{Scheuermeyer, Frank}

frankscheuermeyer@yahoo.ca

ELDERLY patients with primary atrial fibrillation: emergency department management and 1-year outcomes

Scheuermeyer F, Grafstein E, Innes G, Harris D, Stenstrom R, Hunte G, Heslop C, Pourvali R, Christenson J; St. Paul's Hospital and the University of British Columbia, Vancouver, BC

Objectives: Elderly emergency department (ED) patients with atrial fibrillation (AF) have been minimally investigated. We describe the management and 1-year outcomes of ED patients $>75$ years with AF. Methods: This was a retrospective cohort study utilizing the ED administrative database of two urban hospitals to identify consecutive patients $>75$ years old with a primary discharge diagnosis of ECGproven atrial fibrillation from April 1, 2006, to January 31, 2009. Each patient's unique provincial health number was linked to the provincial vital statistics registry, provincial stroke registry, and regional ED database to identify deaths, strokes, and ED revisits within 1 year. Patient comorbidities, rhythms, and treatments were obtained by manual chart review. Patients were stratified into two main groups based on ED presentation and management: rhythm eligible (electrical cardioversion, chemical cardioversion, spontaneous cardioversion) and rhythm ineligible (rate control, no arrhythmia-specific treatment). Primary outcome was the combined 1-year rate of stroke or death; secondary outcomes included the proportion of patients discharged home, stratified by treatment group. Results: A total of 156 consecutive patients $>75$ years old with a primary ED diagnosis of atrial fibrillation were collected, with 52 (33.3\%) eligible for rhythm control. There were 17 deaths and 2 strokes $(12.2 \%, 95 \%$ CI $7.1-17.3 \%)$ in the following year. All deaths and strokes occurred in patients who were ineligible for rhythm control (19 of 104, 18.3\%). The proportions of patients discharged home were $87 \%$ and $72 \%$, respectively, for rhythm-eligible and -ineligible groups. Conclusions: In this two-centre 
cohort of elderly ED patients with primary AF, the majority of patients were discharged home. Patients ineligible for rhythm control had a substantial rate of stroke or death. Keywords: atrial fibrillation, cohort study, elderly patients

\section{Scheuermeyer, Frank}

frankscheuermeyer@yahoo.ca

ONE-YEAR outcomes of patients with atrial fibrillation who are initially managed with oral or intravenous antiarrhythmic agents Pourvali R, Heslop C, Grafstein E, Harris D, Hunte G, Stenstrom R, Christenson J, Innes G, Scheuermeyer F; St. Paul's Hospital and the University of British Columbia, Vancouver, BC

Objectives: Emergency department (ED) patients with atrial fibrillation (AF) who are managed with oral or intravenous antiarrhythmics have not had their long-term outcomes investigated. Methods: This was a retrospective cohort study utilizing the ED administrative database of two urban hospitals to identify consecutive patients with a primary discharge diagnosis of ECG-proven atrial fibrillation from April 1, 2006, to March 31, 2009. Patients receiving oral or intravenous antiarrhythmics as the initial method of rhythm control were identified. Each patient's unique provincial health number was linked to the provincial vital statistics registry, provincial stroke registry, and regional ED database to identify deaths, strokes, and ED revisits within 1 year. Patient comorbidities were obtained by manual chart review. Primary outcome was the combined 1-year rate of stroke or death; secondary outcomes were the proportion of patients converting to sinus rhythm with antiarrhythmic therapy and the proportion discharged home. Results: A total of 112 consecutive patients with a primary ED diagnosis of atrial fibrillation and initial treatment with antiarrhythmic agents were collected, with no deaths of strokes $(0.0 \%, 95 \%$ CI 0.0 $2.6 \%)$ in the following year. Conversion rate was $43 \%$ and discharge rate was $90 \%$. Conclusions: In this two-centre cohort of ED patients with AF who were managed with oral or intravenous rhythm control, no patients died or had a stroke at 1 year. A minority converted to sinus rhythm, but most were discharged home. Keywords: administrative database, antiarrhythmic therapy, atrial fibrillation

\section{Colacone, Antoinette}

eddy.lang@albertahealthservices.ca

STEPS toward alleviating boarding in the emergency department: evaluation of hospital bed occupancy appropriateness

Marc Afilalo, MD, Jean-François Boivin, MD, ScD, Emmanuelle Jourdenais, MD, Stephen Rosenthal, MD, MSc, Nathalie Soucy, PhD, Xiaoqing Xue, MSc; Jewish General Hospital, Emergency Department, McGill University, Montreal, QC

Introduction: Timely access to hospital beds is crucial for emergency departments (ED) to avoid boarding. Bed accessibility is compromised when long-stay patients inappropriately occupy acute care beds. This study aims to identify characteristics and needs of long-stay patients and explore alternative health care resources best satisfying their needs. Methods: Patients hospitalized for $30( \pm 3)$ days were enrolled in two teaching hospitals from March 2009 to April 2010. Obstetric, psychiatric, palliative, and long-term care (LTC) patients were excluded. Data on sociodemographic, medical, and patient needs were collected through chart review and inquires to medical/nursing staff. Appropriateness of hospitalization on day 30 was assessed using the Appropriateness Evaluation Protocol (AEP). Results: A total of 941 patients were recruited with $84 \%$ admitted from the ED. Mean age was $73 \pm 15$ years, $56 \%$ were admitted to medicine, $31 \%$ to surgery, and $13 \%$ to geriatrics. At day 30, the most frequent underlying medical problems were cancer (19\%), infection (19\%), gastrointestinal $(17 \%)$, and neurologic $(17 \%)$ diseases. Based on AEP, $22 \%(n=211)$ of hospitalizations at day 30 were deemed inappropriate due to discharge planning delays $(41 \%)$, lack of alternative resources $(31 \%)$, need for more tests (19\%), psychosocial issues (14\%), lack of outpatient treatment facilities $(13 \%)$, awaiting placement $(14 \%)$ in LTC, palliative care, or rehabilitation institutions. Alternative resources evaluated to better meet patients' needs included LTC $(48 \%)$, rehabilitation $(17 \%)$, palliative $(11 \%)$, and home care $(8 \%)$. Subsequent evaluation using more stringent criteria revealed that more patients could be discharged to alternative facilities such as LTC (eg, stable patients who only needed injection). Conclusions: Over $20 \%$ of long-stay patients were deemed inappropriate, the majority due to discharge planning delays and lack of alternative resources. LTC, rehabilitation, and palliative care were identified as optimally facilitating discharge, thereby increasing bed accessibility for ED. Keywords: appropriateness evaluation protocol, bed utilization, long-term care

Reynolds, Megan

megan.reynolds@utoronto.ca

PHYSICIAN and nurse perceptions in using analgesia protocols: exploring barriers to care

Megan Reynolds, Stella Yiu, Maxwell Smith, Steven Marc Friedman, Tamara Arenovitch, Caesar Lim, Paul Ellis, and David Carr; University Health Network, Toronto, ON

Introduction: Patients presenting with pain to the emergency department (ED) often receive delayed or inadequate analgesia. Use of our institution's triage-driven pain protocol was associated with improved time to analgesia in renal colic patients, but uptake of the protocol was low. We sought to identify barriers to its implementation. Methods: A paper-based survey of full-time staff physicians (MDs) and nurses (RNs) at an urban teaching hospital was conducted using Dillman methodology. Questions interrogated attitudes toward ED analgesia and a triage-driven pain protocol, exploring organizational, social, and professional barriers to its use. Responses were in the form of a 5-point Likert scale, multiple-choice, and free text. Results: The response rate was $53.2 \%$ (84 of 158 ), with $89.5 \%$ (34 of 38) of MDs and $41.7 \%$ (50 of 120) of RNs responding. Both groups felt strongly that employing a pain protocol is efficient in ED analgesia and protocols should be standard of care for common conditions. Neither group felt that a pain protocol would increase workload, facilitate drug-seeking behaviour, or lead to misdiagnosis. RNs were more likely than MDs to feel that a protocol should start only after physician assessment $(p=.0360)$. MDs were less likely than RNs to feel that protocols are an unnecessary change from their prior practice $(p=.0390)$. MDs were more likely than RNs to feel that a reminder $(p=.0106)$ or automatic protocol generation within the chart $(p=.0471)$ would increase use. Neither group felt that chart audits of protocol implementation would increase its use. Conclusions: Clinicians endorsed the use of pain protocols in the ED. We identified differences in opinion toward protocol use and activation among clinician groups. RNs were significantly more likely than MDs to feel that protocols should not start until physician assessment. Addressing clinician-specific barriers might increase the use of pain protocols and expedite analgesia delivery in the ED. Keywords: knowledge translation, pain protocols, quality improvement

McLeod, Shelley L

shelley.mcleod@lhsc.on.ca

AMBULATORY visits to a Canadian emergency department (AVCED)

Woolfrey K, Meiwald A, McLeod SL; Division of Emergency Medicine, Faculty of Medicine, The University of Western Ontario, London, ON

Introduction: The use of emergency departments (EDs) by patients with nonurgent medical concerns is a well-known issue in the Canadian health care system. However, the reasons for choosing the ED as 
a patient's source of care in Canada are not well established. The primary objective of this study was to determine the reasons why patients choose the ED as their health care facility for nonurgent medical care. Methods: A prospective study was conducted over a 2month period in the ED of an academic tertiary care centre (annual census 65,000). All adult patients ( $\geq 18$ years) assigned a Canadian Triage and Acuity Scale (CTAS) score of 3, 4, or 5 were approached by trained research assistants to complete a 19 -item paper-based questionnaire. Patients were excluded if they arrived by ambulance, were cognitively impaired, or had a presenting complaint that was psychiatric in nature. Results: A total of 1,178 patients completed the questionnaire. Mean (SD) age was 40 (17.3) years, and 53.7\% were female; $477(40.5 \%)$ patients were assigned a CTAS score of 3 , while 666 (56.5) were assigned a CTAS score of 4 . Although $85.2 \%$ stated they had a family doctor, $59.7 \%$ felt a family doctor could not deal with the concern, while only $14.6 \%$ felt they could. The remainder were unsure; $44.8 \%$ stated they could be seen by their family doctor within 3 days. Less than $30 \%$ identified a family doctor, walk-inclinic, or urgent care centre as an alternative for care. A total of 513 $(43.5 \%)$ of respondents felt their concern was very urgent or lifethreatening. The majority $(54.1 \%)$ of patients had some college or university education. With regard to perceived seriousness of complaint, no differences were found between respondents with postsecondary education compared to those with high school education only. Conclusion: Although the majority of patients in this study were well educated and had a family doctor and wait times were minimal, the perceived seriousness of a low triage complaint played a major role in the decision to choose the ED as the source of care. Keywords: lowacuity patients, severity perception, survey

McLeod, Shelley L

shelley.mcleod@lhsc.on.ca

\section{ANALYSIS of paramedic error on ambulance call reports}

Stephanie Romano, Matthew Davis, Adam Dukelow, Mike Lewell, Severo Rodriguez; Southwest Ontario Regional Base Hospital Program, London, ON

Introduction: In our emergency medical services (EMS) system, delegation of medical acts for paramedic practice is facilitated by base hospital quality assurance (QA) activities. Retrospective ambulance call evaluations (ACEs) are completed for all protocol-driven prehospital medical directives that are audited as having an error present. Errors considered "critical" or "major" result in remediation, deactivation, or eventual decertification. The objectives of this study were to identify the proportion of errors eventually attributable to documentation and to determine if services utilizing electronic ambulance call records (eACRs) had different documentation error rates compared with services with paper ACRs. Methods: A retrospective study between January 1 and June 28, 2010, was undertaken. The QA process starts with electronic filtering that identifies potential variations from protocols. Auditors used ACEs to determine the potential severity of the error (none, minor, major, or critical). Major and critical errors resulted in further investigation (written response or interview). Professional standards specialists analyzed the ACE, ACR, and responses to determine whether errors were related to documentation or actual medical care. Results: A total of 4,557 ACRs were audited; $342(7.5 \%)$ ACRs were determined to have an error, and $167(49 \%)$ were eventually categorized as documentation errors. Of the 167 charts with documentation errors, $89(53 \%)$ were initially thought to be critical or major errors. After the completed audit response, 86 documentation errors $(96.6 \%)$ were reduced to minor or none. There was no difference $(p=.23)$ in the documentation errors between the eACRs $(89 ; 53 \%)$ and the paper ACRs $(78 ; 47 \%)$. Conclusion: A large proportion of errors were eventually deemed related to documentation and were downgraded in severity. There was no difference in documentation errors rates between electronic and paper ACRs. Keywords: audit, EMS, patient safety

Varner, Catherine E

catherine.varner@gmail.com

\section{A SURVEY of practice patterns of one CCFP-EM program}

Catherine Varner, Howard Ovens, Eric Letovsky, Bjug Borgundvaag; Department of Emergency Medicine, Mount Sinai Hospital, Toronto, $\mathrm{ON}$

Introduction: The third-year College of Family Physicians of Canada Emergency Medicine program (CCFP-EM) was designed for family medicine graduates to develop special competence in emergency medicine (EM). The disposition and practice settings of graduates of the program are largely unknown. The primary objective of this study was to determine the practice settings of graduates of the University of Toronto CCFP-EM program. A secondary objective was to determine the amount and type of additional clinical and nonclinical activities in which the CCFP-EM graduates participate. Methods: Using the Dillman method, the program's 146 CCFP-EM graduates from 1982 to 2009 were surveyed electronically. Respondents were directed to a Web-based survey regarding details of their clinical and academic work since graduation. Additional demographic data were also collected. Results: Of 146 graduates surveyed, 88 responded (response rate of $66 \%$ ). All of the respondents indicated that they had practiced EM at some point after completing the University of Toronto CCFPEM program, and at survey completion, $44.3 \%$ were practicing EM full-time. Of all respondents, $79.8 \%$ remained practicing some EM. Of the EM practicing cohort, $93.8 \%$ worked in urban/suburban hospitals as opposed to rural settings. Those practicing EM expressed high levels of job satisfaction, with $83.3 \%$ reporting 8 or higher on a 10 -point satisfaction scale. The majority of U of T CCFP-EM graduates had participated in a leadership activity in EM (57\%) on local, provincial, or national levels. Conclusions: This survey reports that the majority of $U$ of T CCFP-EM graduates continue to practice EM, and the majority of graduates practice in urban and suburban environments. The low attrition rate of CCFP-EM graduates should be regarded as a success of the CCFP-EM program, and the geographic distribution of all physicians, including EM providers, warrants further study to help plan future physician resources in Canada. Keywords: CCFP-EM, practice patterns, survey

\section{Prendergast, Patrick}

patrick.prendergast@gmail.com

ACUTE appendicitis: optimal outer appendiceal diameter cutoff in a pediatric population

Patrick Prendergast, Dr Tim Lynch, Dr Scott McKillop, Dr. Naveen Poonai, Dr Rodrick Lim; The University of Western Ontario, Schulich School of Medicine and Dentistry, London Health Sciences Centre, Department of Pediatric Emergency Medicine, London, ON

Introduction: In adult populations, an outer appendiceal diameter $(\mathrm{OAD})>6 \mathrm{~mm}$ on ultrasound is generally believed to suggest acute appendicitis. One study of 70 children concluded that an OAD $>6$ $\mathrm{mm}$ has the same accuracy in children as in adults. Another small study in Korea proposed an optimal maximal outer diameter of 5.87 $\mathrm{mm}$ for pediatric patients. Thus, a paucity of evidence-based support for the $6 \mathrm{~mm}$ rule in a pediatric population exists. This study will attempt to determine the optimal cutoff for appendectomy using only the OAD in a pediatric population. Methods: A retrospective review was performed for all urgent abdominal and pelvic ultrasounds performed between July 1, 2008, and June 30, 2010, at the Pediatric Emergency Department of the London Health Sciences Centre. The diagnostic accuracy of various mm cutoff points was assessed; as well, an optimal cutoff point was obtained via ROC curve analysis. 
Results: The OAD was measured in 422 of $688(61.3 \%)$ children. Repeat examinations were excluded, leaving a study sample of 398 children. This sample was divided into a surgical $(n=174)$ and a nonsurgical group $(n=224)$. The sensitivities (SE) and specificities (SP) for various cutoffs were (SE/SP): $(99.3 / 25.1) \geq 4.5 \mathrm{~mm},(98.6 / 33.7) \geq$ $5 \mathrm{~mm},(98.6 / 47.1) \geq 5.5 \mathrm{~mm},(97.9 / 63.1) \geq 6 \mathrm{~mm},(93.7 / 73.3) \geq 6.5$ $\mathrm{mm},(91.6 / 83.9) \geq 7 \mathrm{~mm}$. Weighting sensitivity and specificity equally at $1: 1$, an optimal cutoff of $7.0 \mathrm{~mm}$ was obtained. Discussion: This study confirms the applicability and importance of using the OAD in the pediatric population. Exchanging decreasing sensitivity with increasing specificity and vice versa, no "ideal" cutoff exists. ROC curve analysis, which balances SE and SP, suggests that $7 \mathrm{~mm}$ is most appropriate. Adopting a $7 \mathrm{~mm}$ cutoff rather than $6 \mathrm{~mm}$ cutoff in clinical practice would lead to greater numbers of missed cases of acute appendicitis but would reduce the number of unnecessary surgeries. Perhaps a more useful framework would be to designate an $\mathrm{OAD}<6$ $\mathrm{mm}$ as negative, an OAD 6-7 $\mathrm{mm}$ as equivocal, and an OAD $>7 \mathrm{~mm}$ as positive. Keywords: outer appendiceal diameter, pediatric appendicitis, ultrasound

Stang, Antonia AS

antonia_stang@hotmail.com

QUALITY indicators for high-acuity pediatric conditions requiring emergency care

Antonia S. Stang, MD, MBA, MSc, Astrid Guttmann, MD, MSc, Sharon Straus, MD, MSc, David Johnson, MD; Department of Pediatrics, University of Calgary, Calgary, AB

Introduction: Identifying gaps in care and improving outcomes for high-acuity pediatric conditions require the development of relevant, evidence-based performance measures. The objective of this project was to use a systematic process involving multiple stakeholders to develop evidence-based quality of care indicators (QI) for high-acuity pediatric conditions relevant to any emergency department (ED) setting where children are seen. Methods: Data on the main diagnosis for high-acuity (resuscitation and emergent at triage) pediatric patients (age 0-19 years) seen in all EDs in Ontario and Alberta (together representing $50 \%$ of Canada's population) were collected to construct an initial list of potential conditions. A prioritized list of conditions was selected by a 32-member advisory panel based on three criteria: importance (morbidity or mortality), impact (potential to address gap between current and best practice), and validity (adequacy of scientific evidence linking performance of care to patient outcome). A systematic review of the literature was conducted for the selected conditions to identify existing quality indicators and practice guidelines, high-quality systematic reviews, and randomized controlled trials (RCTs) that could be used to inform the creation of quality indicators. Modified Delphi methods consisting of two rounds of anonymous questionnaires and a face-to-face meeting of an expert panel were used for final indicator selection. Results: The conditions selected by the advisory panel for indicator development were diabetic ketoacidosis, status asthmaticus, anaphylaxis, status epilepticus, severe head injury, and sepsis. Results from the final indicator selection will be presented. Conclusions: This rigorous process adds to the existing body of evidence-based quality of care indicators relevant for the ED management of pediatric patients. Future work will test the reliability and feasibility of data collection on these QI across the spectrum of ED settings that provide care for children. Keywords: high acuity, pediatrics, quality indicators

Bond, Chris

cmbond@ualberta.ca

THE EMERGENCY to Home Project: impact of an emergency department care coordinator on home care referrals and hospitailization among seniors
Bond, CM, Choo, Q. Davenport, J. Taylor, A. Lee, V. Lang, E; University of Calgary, Calgary, $\mathrm{AB}$

Introduction: Seniors make up between 14 and $21 \%$ of all emergency visits but are disproportionately larger users of ED care and inpatient resources. Emergency department care coordinators (EDCCs) target atrisk seniors and connect them to appropriate community services. The goal of this study was to determine whether implementing new EDCCs and expanding the role of already existing EDCCs would increase the number of seniors' home care referrals and decrease admission rates. Methods: This is a pre-post study performed in four EDs in three Alberta cities. All seniors aged 65 and over who visited the ED during 2010 study dates corresponding to EDCC presence were enrolled. At two of the sites, increased support for existing EDCCs was implemented in the form of clerical support workers and transition coordinators. At the other two sites, new EDCC positions were created. The primary outcomes were the number of home care referrals and seniors' admission rates; this was compared to baseline data from 2008/2009. Chi-square and Student $t$-test were employed to compare pre and post outcomes. Results: In 2008/2009, there were 100,429 seniors' ED visits at the four sites. During the study, 625 of 4,010 (15.6\%) seniors assessed at the Misericordia Community Hospital (MCH) were discharged with home care referrals compared with a previous baseline rate of $0.5 \%$. At the Red Deer Regional Hospital, there was a similar increase in homecare referrals $(22.7 \%$ v. $6.4 \%)$. Admission rates did not change from the pre to post study period. Conclusions: The implementation and expanded role of EDCCs have increased the number of seniors being discharged with home care referrals; however, they have not resulted in decreased seniors' hospital admissions. Keywords: geriatrics, home care referrals, nurse practitioners

Howlett, Michael K mhowlett@tru.eastlink.ca

RELIABILITY of Canadian Emergency Department Triage Acuity Scale (CTAS) triage: a paramedic-nurse triage comparison

Michael K. Howlett, MD; Department of Emergency Medicine, Dalhousie University, and Department of Emergency Medicine, Horizon Health Network, Saint John Zone, Saint John, NB

Introduction: We evaluated interrater reliability of paramedic-performed Canadian Triage and Acuity Scale (CTAS) triage compared with nurse triage. Method: An emergency nurse reviewer (EN) retrospectively evaluated nurse (N) CTAS scores on 1 month's ED charts blinded to physician data. The ED gave CTAS training to paramedics (primary care paramedic level) (P). After 6 months, interrater reliability (using quadratic weighted kappa) was performed on 1 month's $\mathrm{P}$ charts. Results: There were 3,091 (N), 2,787 (P) patients: CTAS $(\mathrm{N}): 1$, $0.23 \% ; 2,4.4 \% ; 3,39 \%, 4,49 \%$, and $5,5.5 \%$. CTAS (P): $1: 9.3 \% ; 2$ : $6.6 \% ; 3: 47 \% ; 4: 42 \% ; 5: 4 \%$. There were insufficient data in 1,559 $(50 \%)$ v. $521(19 \%)$. Inconsistent triage was $16 \%$ v. 31\%. Weighted kappa was 0.604 , or good agreement $(\mathrm{N})$, and $0.63(\mathrm{P})$ (absolute kappa $0.49,0.53$ ). Clinical indicators (insufficient data, pain scale, chest pain, traumatic injury, respiratory illness, and headache) captured $62 \%$ and $74 \%$ of cases (pain scale score: 858 [30.9\%] versus 1,895 [72\%]). Discussion: Clinical indicators captured over $70 \%$ of visits. P training produced a "good" level of interrater agreement. Ps performed favourably, capturing triage, clinical indicators, and pain scales, but were inconsistent with EN twice as often. Discrepancy between good data availability and kappa $(\mathrm{P})$ versus a worrisome doubled percentage in absolute triage inconsistency suggests a need for better measures of interrater reliability beyond kappa or that moderate kappa values are not "good enough," without comparing outcomes. The study was retrospective and did not consider accuracy from an outcomes perspective. Conclusion: Paramedics and nurses performed CTAS triage with similar interrater reliability. The inability of kappa to differentiate between differing levels of 
triage inconsistency requires further study and more emphasis on outcomes. Keywords: CTAS, interrater reliability, paramedics

Carter, Kyle W

kcarter2011@meds.uwo.ca

PHYSICIAN perceptions of barriers to patient care in southwestern Ontario rural emergency departments

K. Carter, HBMSc, K. Cassidy, HBSc, and M. Bhimani, CCFP (EM); The University of Western Ontario, London, ON

Introduction: We sought to determine the perceptions of physicians staffing rural emergency departments (EDs) in southwestern Ontario with respect to factors affecting patient care in the domains of physical resources, available support, and education. Methods: A confidential 30 -item survey was distributed to ED chiefs at 16 rural EDs throughout southwestern Ontario during the month of July 2010. Using a 5-point Likert scale, physicians were asked to rate their perception of various factors that affect patient care in their ED. Demographic and practice characteristics were also collected to characterize the participating centres and physicians. Results: Responses were received from 13 $(81.3 \%)$ of the surveyed EDs. The majority of our respondents working in rural emergency departments were CCFP physicians with ATLS and ACLS training. Crowding from inpatient boarding (mean Likert score of 2.31) and inadequate physician staffing/coverage in EDs (mean 2.67) were identified as having the greatest negative impact on patient care in rural southwestern Ontario EDs. Access to information sharing within hospital (mean 4.35), access to emergent laboratory studies (mean 4.29), and access to medications in the ED (mean 4.23) were identified as having the greatest positive impact on patient care within the rural EDs sampled. Conclusions: Our survey shows that physicians practicing emergency medicine in southwestern Ontario perceive that crowding and adequate staffing of the EDs by physicians are the greatest barriers to providing patient care. Furthermore, the survey identified factors in rural emergency departments, which are perceived to have a positive impact on patient care, such as access to information sharing, emergent laboratory studies, and access to medications within the ED. Keywords: emergency overcrowding, rural EM, survey

Thiruganasambandamoorthy, Venkatesh

pondyvenki@yahoo.com

FREQUENCY of visits and outcomes in the emergency department nontraumatic low back pain patients

Ekatrina Turko, Venkatesh Thiruganasambandamoorthy, Dominique Ansell, George A. Wells, Ian G. Stiell; Department of Emergeny Medicine, University of Ottawa, Ottawa, ON

Introduction: The extent of ED visits due to nontraumatic low back pain (LBP) and the proportion of patients with serious underlying pathology are not known. The aim of this study was to evaluate the frequency, management, and outcomes of ED nontraumatic LBP patients. Methods: In this health records review, we screened consecutive patient visits to two tertiary care EDs over a 3-month period. We included adults with a primary complaint of nontraumatic LBP (below the costal margins and above the buttocks). We excluded patients who were not from the local area and those with pain consistent with previously diagnosed urinary calculi. We extracted patient characteristics, ED management, disposition and outcomes (compression fractures, osteomyelitis, spinal/paraspinal abscess, malignancy, cauda equina syndrome, or paraspinal pathology such as abdominal aortic aneurysm). A 30-day follow-up was done at all local adult hospitals. We conducted descriptive analyses with $95 \%$ CIs. Results: Of the 698 visits screened, 330 patients were included with these characteristics: mean age 49.2 years (range 17-93), 50.9\% females, previous cancer $6 \%$, on anticoagulants $3.9 \%$, immunosuppression $2.4 \%$, osteoporosis $1.8 \%$, and arrival by ambulance $25.5 \%$. Of these, $43.9 \%$ were investi- gated: blood test $27.3 \%$ and imaging $33.9 \%$ (x-ray $17.2 \%$, ultrasound $2.7 \%$, CT $7.6 \%$, MRI 3.9\%), and 3\% (95\% CI 1.7-5.5\%) were admitted. Most common final ED diagnoses were mechanical LBP $49.1 \%$, sciatica $12.1 \%$, and LBP NYD $8.8 \%$. Outcomes occurred in $10 \%$ of patients (fractures $3 \%$, osteomyelitis $0.9 \%$, spinal abscess $0.3 \%$, malignancy $1.2 \%$, cauda equina syndrome $0.3 \%$, disk prolapsed requiring surgery $3.0 \%$, and paraspinal infections $1.2 \%$ ), with $3.3 \%$ detected during the ED visit and $6.7 \%$ after ED discharge. Conclusions: This is the first study to evaluate ED nontraumatic LBP. A significant proportion of patients have serious underlying etiology detected after ED discharge. Development of a clinical decision rule to guide investigation of ED LBP patients will be very useful. Keywords: clinical decision rule, health records review, low back pain

Vaillancourt, Christian

cvaillancourt@ohri.ca

REVIEW of in-hospital cardiac arrest care processes and evaluation of time delays amenable to automated external defibrillator use by allied health professionals

Waleed Chehadi, Christian Vaillancourt, Michael Gatta, Rina Marcantonio, Redouane Bouali; Department of Medicine, University of Ottawa, Ottawa, ON

Introduction: Most in-hospital defibrillators are equipped with an automated external defibrillator (AED) mode, yet nurses and respiratory therapists (RT) trained in their use are seldom authorized to use them. We sought to determine our ability to collect reliable in-hospital cardiac arrest data and evaluate time delays amenable to AED use by nurses and RTs. Methods: We reviewed in-hospital cardiac arrest health records at two campuses of a teaching hospital (130,000 ED visits/year). A health record analyst determined the total number of inpatient deaths (including newborn, excluding stillbirth) over the study period. RTs are always involved during resuscitation efforts, so we used their patient care records to identify cardiac arrest cases where resuscitation was attempted. Two investigators reviewed the records using a standardized Utstein tool for reporting on in-hospital cardiac arrest and had previously trained together until reaching agreement on all data element definitions. We reviewed 4 months in 2009 using paper records and 3 months in 2009-2010 using new electronic records. Results: There were 746 in-hospital deaths over the study period, most of which had a do not resuscitate order. Among the 152 patients requiring urgent $\mathrm{RT}$ intervention, 30 were cardiac arrest patients for whom resuscitation was attempted. They were median age $70,67 \%$ male, $43 \%$ and $40 \%$ from medicine ward and ICU, $70 \%$ witnessed, $57 \%$ monitored, $40 \%$ respiratory, $32 \%$ PEA, $28 \%$ asystole, $20 \% \mathrm{VF} / \mathrm{VT}, 80 \%$ required CPR, $63 \%$ had ROSC, $53 \%$ lasted between 20 minutes and 24 hours, $7 \%$ received therapeutic hypothermia and later died, $13 \%$ survived to hospital discharge. Median time interval between arrest and first shock was 9 minutes. Conclusions: Unlike prehospital services, only rare hospitals maintain a cardiac arrest registry. We identified a long delay before shock administration and demonstrated our ability to collect reliable in-hospital cardiac arrest data and monitor the implementation of an AED program that could decrease the time to first shock. Keywords: allied health professionals, automated external defibrillators, in-hospital cardiac arrest

Del Bel, Nicole

nicole.delbel@medportal.ca

NEEDS assessment for continuing education for emergency medicine physicians

N. Del Bel, D. Lin, and J Sollazzo; McMaster University, Hamilton, $\mathrm{ON}$

Introduction: Currently, continuing medical education (CME) is a significant component of maintaining skills, knowledge, and certifica- 
tion for working in the emergency department. The objective of this study is to ascertain whether physicians who practice emergency medicine would be interested in, and receptive to, a problem-based smallgroup module format for CME. We aim to assess the current perceived needs of emergency physicians regarding both the methods and the topics that they would wish to see in future CME programs. Methods: A formal perceived needs assessment was undertaken. A survey was distributed to local emergency physicians via e-mail. This was a voluntary survey in which consent was implied on completion of the survey. A small monetary incentive draw among respondents was supplied free from industry sponsorship. Results: There were 144 initiated responses to the survey, 5 of which were incomplete. A third of responses were from residents, a third from those practicing less than 10 years, and a third from those practicing greater than 10 years. Most respondents were from urban areas, with only $16 \%$ practicing in rural environments. The current three most common methods of obtaining CME include conferences, certification courses, and journal clubs. Most felt their preferred method of learning was through evidencebased cases and discussion with colleagues. The perceived largest barrier to CME was time. Currently, less than $50 \%$ partake in small-group CME, and of those, $88 \%$ find it useful. Sixty-five percent of respondents were interested in small-group modules for CME. Most physicians feel that "in-person study groups" would be their preferred method of group CME. Conclusions: Continuing education is important not only for maintaining certification but also disseminating changes to practice based on current evidence. Our needs assessment showed an interest in evidence-based clinical cases and group learning. Keywords: continuing medical education, needs assessment, survey

Lubin, Jonathan M

jonathanmichaellubin@gmail.com

PATTERNS of procedural sedation and analgesia practice in a community teaching hospital

Jonathan Lubin, Sherry Stackhouse, Dr. Gary Andolfatto, and Dr. Elaine Victoria Willman

UBC Undergraduate Medical Program; research performed at Lion's Gate Hospital, North Vancouver, BC

Introduction: Procedural sedation and anesthesia (PSA) is the standard of care for patients undergoing painful procedures in the emergency department (ED). Traditionally, this has been most commonly accomplished using fentanyl-midazolam combination. There has been increasing interest in newer PSA agents, including propofol and ketamine-propofol combinations. We evaluated the patterns of use of pharmacologic agents for PSA at our community teaching hospital over a 5.5-year period. Methods: Prospective data were collected on all PSA events occurring in the ED of a 250-bed community teaching hospital and level 3 trauma centre between July 2005 and November 2010. Descriptive statistical analysis was performed on patient demographics, ASA class, comorbidities, procedure performed, and PSA regimen chosen. Results: Over the study period, prospective data were collected on 2,021 PSA events. Procedures performed included fracture reduction (42\%), cardioversion (24\%), joint dislocations (13\%), incision and drainage (9\%), chest tube insertion $(4 \%)$, and lacerations (4\%). PSA regimens chosen included ketamine-propofol (63\%), propofol alone (29\%), and ketamine alone (3\%). Fentanyl-midazolam combinations were chosen in $0.01 \%$ of PSAs. Patients receiving ketaminepropofol most commonly underwent orthopedic procedures $(69 \%)$ and had a mean age of $42 \pm 24.9$ years, with $45 \%$ being female. Patients receiving propofol alone most commonly underwent cardioversion $(59 \%)$, with a mean age of $56 \pm 20.6$ years $(40 \%$ female). Patients receiving ketamine alone had a mean age of 9 years (36\% female), with 55\% undergoing laceration repair. ASA scores were similar among the propofol and ketamine-propofol groups. Conclusions: At our community teaching hospital, the nontraditional agents of propofol and ketamine, used alone and in combination, are the most frequently used drugs for ED PSA. Patients undergoing orthopedic procedures were most likely to receive ketamine-propofol combination, while cardioversion was most commonly performed with propofol alone. Keywords: fentanyl-midazolam, ketamine-propofol, procedural sedation and analgesia

\section{Saunders, Brooke}

brooke.saunders@queensu.ca

INCIDENCE of and characteristics associated with intentional self-harm within the trauma population

Brooke Saunders, Amanda McFarlan, Avery Nathens, Carolyn Snider; Queen's University, Kingston, ON

Introduction: The primary objectives of this study were to determine the incidence of and characteristics associated with intentional selfinflicted injuries within an urban Canadian trauma centre. Methods: This was a retrospective cohort study utilizing St. Michael's Hospital Trauma Registry Database, which tracks demographic data and clinical outcomes for all patients admitted to the SMH Trauma Service. Data abstraction was conducted on all patients admitted with intentional selfinflicted injuries for the fiscal years 2005-2010. Cases were identified using International Classification of Disease (ICD-9) External Cause of Injury codes. Results: During the fiscal years 2005-2010, 4,163 trauma cases were entered into the database, of which $177(4.2 \%)$ were due to intentional self-injury. The majority of cases were male $(65.0 \%, n=$ 117 ), with an average age of $39.7 \pm 2.6$ years and an average ISS of $17.8 \pm 2.2$. The leading method of injury was stabbing (48\%), followed by jumping (40\%) and gunshots (4\%). Women accounted for a higher proportion of the stabbings and jumpers and a lower proportion of gunshots compared to men. Women also had a significantly lower ISS $(14.2 \pm 3.6)$ than men $(19.6 \pm 2.8)$, as well as a lower average age (35.0 \pm 3.2 years $)$ than men $(42.0 \pm 3.5$ years $)$. Over half of the patients had a documented psychiatric medical history; however, only $81 \%$ of cases received psychiatric consultations while in hospital. Four individuals were admitted to the trauma service with self-inflicted injuries more than once, two of whom died as a result of their second self-inflicted injury. The average total length of stay was $18.9 \pm 3.6$ days, with an average of $5.9 \pm 1.4$ days spent in the ICU. In-hospital deaths accounted for $15 \%$ of cases. Conclusion: The 5-year incidence of intentional selfharm within the SMH trauma centre was $4.2 \%$. There were significant differences between men and women with respect to ISS scores and age. Keywords: health records review, intentional self-harm, trauma

\section{Atkinson, Paul}

Paul.Atkinson@HorizonNB.ca

\section{HEAD injury management around the clock}

Paul Atkinson, Erin Sproul, Sue Benjamin, Alysha Mehta; Saint John Regional Hospital, Saint John, NB

Introduction: We aimed to determine whether the time of day, the availability of radiology staff, and the severity of the injury affected the time to CT scan of patients with head injury presenting to the emergency department at a level 1 trauma centre. Methods: The study was a retrospective database and chart review, with preliminary data collected on 171 patients presenting with head injury to the emergency department over a 13-month period. Our primary outcome was time from emergency physician assessment to CT scan. Data were analyzed according to severity of head injury, time of presentation, and availability of on-site radiology support using nonparametric tests (GraphPad Prism). Results: Time to CT did not vary significantly with severity of head injury; median times to CT were $69.5,65$, and 50 minutes for patients presenting with mild (GCS 13-15), moderate (GCS 9-12), or severe (GCS (3-8) head injuries, respectively ( $p=$ .0674). There was a statistically significant delay to CT scan when the 
CT technologist was not present in the hospital with a median time of 73.5 minutes compared to 66 minutes when present $(p=.0391)$. There was also a significantly longer time to CT scan overnight (median 77 minutes) when compared to day and evening presentations (median 66 minutes; $p=.0328$ ). Conclusion: Absence of a CT technologist and requesting a scan during the overnight hours were associated with a significantly increased time in obtaining a CT head scan for head injury. There did not appear to be a significant difference between different severity of head injury. Further investigation is indicated to determine if the main effect is due to the time of day of the scan request or the absence of an on-site CT technologist. Keywords: head injury, neuroimaging, time to $\mathrm{CT}$

\section{Rowe, Brian $\mathrm{H}$}

brian.rowe@ualberta.ca

SEVERE sepsis and septic shock in Canadian emergency departments: have optimal guidelines translated into optimal diagnosis and monitoring?

Gaudet J,* Djogovic D, Singh M, Villa-Roel C, Rowe BH; *University of Calgary, Calgary, $\mathrm{AB}$, and University of Alberta, Edmonton, $\mathrm{AB}$

Introduction: Proven therapies and evidence-based guidelines exist for the diagnosis and treatment of severe sepsis and septic shock. The objective of this study was to characterize the influence these advances have had on the diagnostic strategies utilized by Canadian emergency department (ED) physicians. Methods: A national electronic survey was distributed to CAEP physicians using a modified Dillman technique. Physicians were asked to complete an anonymous questionnaire about their diagnostic approaches and management of patients with suspected severe sepsis and septic shock. Statistics are descriptive and a priori academic and nonacademic subgroups are compared using chisquare tests. Results: Of 965 physicians surveyed, $290(30 \%)$ responded; $168(76.4 \%)$ were male, and the highest proportion of respondents $(43 \%)$ were aged $35-44$. Most (49\%) reported completing 11-15 clinical shifts per month, and almost all (>95\%) worked in hospitals with surgical and intensive care unit capabilities. Physicians reported measurement of lactate levels more often in the setting of hypotension $(<90 \mathrm{~mm} \mathrm{Hg})$ than when systolic blood pressures exceeded $90 \mathrm{~mm} \mathrm{Hg}(80 \%$ v. 50\%; $p<.001)$; no differences existed between academic v. nonacademic centres. Most respondents $(90 \%)$ reported measurement of random cortisol levels $<25 \%$ of the time. Upper limb central venous lines (CVLs) were more commonly placed than femoral CVLs $>75 \%$ of the time $(26 \%$ v. $6 \% ; p<.001)$. Physicians in academic and nonacademic centres performed similarly with regard to placement of upper limb CVLs; however, academic centres utilized ultrasound more frequently to guide CVL insertion $(49 \% \mathrm{v}$. $33 \% ; p=.063$ ). Conclusions: Canadian ED physicians report that they measure lactate levels routinely in hypotensive patients with suspected severe sepsis and septic shock. A practice gap exists in several other aspects of sepsis care, particularly regarding upper limb CVL placement. Further research to understand the barriers impeding evidencebased ED sepsis care is needed. Keywords: central venous access, guideline compliance, sepsis

\section{McLeod, Shelley L}

\section{shelley.mcleod@lhsc.on.ca}

A COMPARISON of CTAS scoring between paramedics and triage nurses: a retrospective review

Thivian Vandeyar, Andrea Sereda, Mike Lewell, Adam Dukelow, Shelley McLeod, Matthew Davis, Severo Rodriguez; Division of Emergency Medicine, Faculty of Medicine, The University of Western Ontario, London, ON

Introduction: The Canadian Triage and Acuity Scale (CTAS) is an objective tool that enables emergency departments (EDs) to prioritize patient care by triaging patients according to the severity of their presenting signs and symptoms. The CTAS system is also used by emergency medical services (EMS) and EDs to communicate patient acuity and allot the most appropriate resources accordingly. For this system to succeed, there needs to be reliability of the CTAS scoring between EMS and the ED. The objective of this study was to assess the interrater reliability of CTAS scores provided by EMS with those of the triage nursing staff (RNs) and ED physicians. Methods: A retrospective medical record review was conducted for patients $\geq 18$ years brought to the ED via EMS over a 6-month period. The CTAS scores were obtained from the ambulance call record (ACR) and the nursing triage record. Interrater reliability between the scores was calculated. The patient's chief complaint and vital signs were also obtained from the ACR and nursing triage record. These data were used to generate retrospective ED physician CTAS scores. The ED physician scores were compared with those established by EMS and RNs to assess interrater reliability. Results: A total of 101 charts were reviewed. The EMS CTAS scores and RN CTAS scores were in agreement in $73.0 \%$ of the charts reviewed $(\kappa=0.56,95 \%$ confidence interval $[\mathrm{CI}] 0.42$ 0.70). The EMS CTAS score compared with the ED physician CTAS score showed $69 \%$ agreement $(\kappa=0.52,95 \%$ CI $0.38-0.66)$. The RN CTAS score compared with the ED physician CTAS score showed $84.2 \%$ agreement $(\kappa=0.75,95 \%$ CI $0.64-0.86)$. Conclusions: CTAS scoring by EMS showed only moderate agreement with scoring by $\mathrm{RNs}$ or the retrospective ED physician scoring. The RN scoring had substantial agreement with the ED physician scoring. Optimal use of the CTAS system requires greater consistency between EMS and staff of the ED when assigning scores. Continued paramedic CTAS training is required to acquire this consistency. Keywords: CTAS, interrater reliability, paramedics

Penciner, Rick

rick.penciner@utoronto.ca

FACULTY and professional development needs of emergency medicine faculty

Rick Penciner, Shirley Lee; Division of Emergency Medicine, Department of Family and Community Medicine, University of Toronto, Toronto, ON

Introduction: Faculty development is emerging as an important trend and driver in emergency medicine (EM) education. However, little is known of the individual needs of EM faculty. The purpose of this study is to determine the faculty and professional development needs of the faculty members in the Division of Emergency Medicine in the Department of Family Community Medicine at the University of Toronto. Methods: An online needs assessment was conducted using a modified Dillman Tailored Design Method. All physicians in the Division of Emergency Medicine in the Department of Family Community Medicine at the University of Toronto $(n=133)$ were invited by e-mail to complete a Web-based 62-item questionnaire. Three e-mail contacts over a 3 -week period were used. The results were analyzed using descriptive statistics. Results: Forty-eight (48) physicians responded to the survey (response rate of $36 \%$ ). The respondents were from six different emergency departments, with $60 \%$ from community teaching hospitals and $40 \%$ from academic health sciences centres. Seventy-one percent were full-time faculty, with $60 \%$ at the rank of lecturer. While $48 \%$ reported that they had attended a faculty development program in the previous 5 years, only $15 \%$ attended a session at the university's Centre for Faculty Development. Forty-one percent reported that they would "very likely" or "probably" attend an EM faculty development session within the next year. The most commonly reported barriers to attending faculty development programs were timing of session $(79 \%)$ and individual time demands $(72 \%)$. Respondents identified needs in the areas of teaching, technology, administrative and career development. The 
most important identified needs were "teaching in a busy ED" $(75 \%)$ and "giving effective feedback" (69\%). Conclusions: EM faculty at the University of Toronto perceive a need for formal faculty and professional development programs. The perceived needs are diverse, as are the barriers to participation. Keywords: faculty development, needs assessment, survey

\section{Touchburn, Andre}

andre.touchburn@gnb.ca

CONTINUOUS quality improvement of cardiopulmonary resuscitation (CPR) in an ambulance service: an evidence-based approach Dr. Andre Touchburn

Centre de formation medicale du Nouveau-Brunswick

Introduction: CPR quality is an important determinant of cardiac arrest outcome. We hypothesize that provision of real-time and retrospective feedback on quality metrics leads to improvement in performance of CPR among approximately 1,000 paramedics. Methods: Real-time performance feedback is provided by the QCPR sensor of the Philips MRX defibrillator. Patient care records and defibrillator data for all out-of-hospital cardiac arrests are compiled and analyzed with regard to CPR quality by continuous quality improvement (CQI) personnel. All paramedics who perform resuscitation receive individual feedback on their performance, including a review of defibrillator waveforms. Global performance targets are addressed with newsletters and regular training sessions, which promote understanding of the physiologic basis of resuscitation protocols. Results: From January 2008 to July 2010, 1,045 nonpediatric, nontraumatic cardiac arrests were identified of a total of 1,173 (incidence 62 of 106 years); median age $66(54,77)$ years, $68 \%$ male. The number of complete records increased from 203 of $390(52 \%)$ in 2008 to 305 of $426(72 \%)$ in 2009 and 180 of $229(79 \%)$ in $2010, p<.01$ (chi-square). In the records available for analysis of CPR quality (764 of 1,045 [73\%]), percentage of time with chest compressions was $70 \pm 12 \%$ with an increase of $5 \%$ per year $(95 \%$ CI 4,6$), p<.01, r^{2}=.071$ (linear regression with case date as independent variable). Average compression depth has been unchanged throughout the registration; $39 \pm 6.7 \mathrm{~mm}$. Among 822 cases of presumed cardiac origin, $66(8 \%)$ patients had prehospital ROSC, and this was similar throughout the registration period. Conclusions: Our comprehensive CQI process has improved accuracy and validity of data collection and enabled detection of weaknesses, implementation of remedial education based on evidence of performance, and monitoring of the effects with regard to CPR quality. We have detected temporal improvement, but further action is needed. Keywords: CPR, CQI, performance feedback

McLeod, Shelley L

shelley.mcleod@lhsc.on.ca

THE UTILITY of emergency department ordered diagnostic tests for acute aortic dissection: a 5-year review

Mahn J, McLeod SL, Theakston KD; Division of Emergency Medicine, Faculty of Medicine, The University of Western Ontario, London, $\mathrm{ON}$

Introduction: Acute aortic dissection is a rare life-threatening condition that often presents atypically mimicking other more common diagnoses. Due to the diagnostic challenges of aortic dissection, the objectives of this study were to evaluate the utility of various radiographic and laboratory investigations. Methods: A retrospective medical record review was conducted of all adult ( $\geq 18$ years) patients with a discharge diagnosis of aortic dissection who presented to a southwestern Ontario emergency department (ED) between 2005 and 2010. Data were extracted by trained research personnel using a standardized data collection tool. Results: Fifty charts were included in this 5-year review. The mean (SD) age was 65.5 (13.4) years, and 54\% were male.
Thirty-nine (78\%) patients were triaged as CTAS 1 or 2 . Three $(6 \%)$ patients had an ED visit in the previous 7 days with a potentially associated symptom (chest pain, abdominal pain, and hypotension). Thirtynine $(78 \%)$ patients had a radiologist-interpreted chest $\mathrm{x}$-ray, of which $28(72 \%)$ were done portably. Eight of $39(20.5 \%)$ were reported as normal, and $20(51 \%)$ showed nonspecific abnormalities. Interestingly, only 11 of $39(28 \%)$ reported a wide or prominent mediastinum. Thirteen of $50(26 \%)$ patients had a D-dimer ordered, and $11(85 \%)$ were elevated. Of the $42(84 \%)$ patients who had a troponin done, $28(67 \%)$ were normal. Conclusions: These results demonstrate the poor diagnostic utility of plain film chest $\mathrm{x}$-ray. While the D-dimer is often elevated, its accuracy is insufficient to be a reliable test for aortic dissection. Emergency physicians should not rely on traditional imaging and laboratory investigations when considering the diagnosis of acute aortic dissection. Keywords: aortic dissection, chest radiography, troponin

\section{Scheuermeyer, Frank}

frankscheuermeyer@yahoo.ca

DECLINE in the incidence and severity of acute coronary syndrome among emergency department patients with potential ischemic chest pain from 2000 to 2006: a prospective analysis

Scheuermeyer F, Wong H, Yu E, Innes G, Grafstein E, Boychuk B, Christenson J; St. Paul's Hospital and the University of British Columbia, Vancouver, BC

Background: Patients presenting to an emergency department (ED) with potential ischemic chest pain have a reported 12 to $25 \%$ acute coronary syndrome (ACS) rate. Although the population rate of ACS has decreased over the past decade, this trend has not been reported among ED patients with potential ischemic chest pain. Methods: Cohorts of ED chest pain patients were prospectively enrolled at a university hospital in both 2000 and 2006. Patients were followed for 30 days to determine the primary outcome of ACS. Both cohorts were stratified into ACS subgroups (STEMI, NSTEMI, and unstable angina) and compared on ECG changes (proportion of patients with predefined ischemic features) and cardiac biomarkers (median initial biomarker elevation). Results: Groups were similar in age, gender, and vital signs. ACS prevalence was $21.4 \%$ (199 of 931) in 2000 and $11.4 \%$ (127 of 1,116$)$ in 2006 (difference $10.0 \%, 95 \%$ CI $6.8-$ $13.3 \%$ ), with all three subtypes decreasing significantly. In the STEMI population, median initial biomarker elevation decreased significantly from $4.35 \mu \mathrm{g} / \mathrm{L}$ to $0.05 \mu \mathrm{g} / \mathrm{L}$. In the NSTEMI group, biomarkers remained constant, as did the proportion of patients with ischemic ECG features (67.7 v. 63.3\%). In UA patients, ischemic ECG changes declined from 49.1 to $25.0 \%$. The proportion of UA patients with normal ECGs and negative biomarkers increased from 50.9 to $72.7 \%$. Conclusions: From 2000 to 2006, the incidence of ED patients with ACS decreased by nearly 50\%. The 2006 ACS patients had fewer ischemic ECG findings, lower biomarker elevations, and a higher proportion of patients with no ECG changes and negative biomarkers. This may make ED diagnosis of ACS more challenging. Keywords: acute coronary syndrome, secular trends, spectrum of illness

Piggott, Zoë S.

zoepiggott@gmail.com

APPLYING lean principles to improve early cardiac care in the emergency department

Dr. Zoë Piggott, Mr. Trevor Strome, Dr. Alecs Chochinov; Emergency Medicine Program, University of Manitoba, Winnipeg, MB

Introduction: To achieve our goal of excellent emergency cardiac care, our institution embarked on a Lean process improvement initiative. We sought to examine and quantify the outcome of this project in 
our emergency department. Methods: Front-line emergency department staff participated in several Rapid Improvement Events, using Lean principles and techniques such as waste elimination, supply chain streamlining, and standard work, to increase the value of the early care we provide to suspected ACS (acute coronary syndrome) patients. A historical chart review was also conducted. To quantify our success, proportions of care milestones (first ECG [electrocardiogram], ECG interpretation, MD [physician] assessment, and ASA [acetylsalicylic acid] administration) meeting target times were chosen as outcome metrics in this before-and-after study. Results: Proportion of 12-lead ECGs completed $\leq 10$ minutes after patient triage increased by $37.35 \%$ $(p<.0001)$. Physician assessments initiated in $\leq 60$ minutes increased by $12.14 \%(p=.0251)$. Times to ECG, MD assessment, and ASA administration also continued to improve significantly over time ( $p$ values $<.0001)$. Post-Lean, median time from ECG performance to physician interpretation was 3 minutes. All of these improvements were achieved using only existing staff and resources. Conclusions: Application of Lean principles can significantly improve attainment of early diagnostic and therapeutic milestones of emergency cardiac care. Keywords: lean processes, quality of care, rapid improvement events

\section{Atkinson, Paul}

Paul.Atkinson@HorizonNB.ca

SERVICE evaluation of emergency ultrasound in a UK emergency department

Paul Atkinson, Richard Kendall, Ivan Wong, Thilina Jayatilleke; Saint John Regional Hospital, Saint John, NB; University of Cambridge, Cambridge, United Kingdom

Introduction: We aimed to describe the number and variety of ultrasound scans performed in the emergency department (ED) of a UK teaching hospital and to assess the accuracy of the scans performed. Methods: We analyzed a database of all examinations performed by emergency physicians in the ED of a tertiary-level UK teaching hospital during an 18-month period. Follow-up were obtained through the hospital information system. Results: A total of 532 scans were performed by 57 emergency physicians over a 77-week period. The majority were level 1 scans: 169 (32\%) focused assessments by sonography in trauma (FAST), $155(29 \%)$ abdominal aortic aneurysm (AAA) screening scans, $27(6 \%)$ basic chest ultrasound scans for pleural and pericardial fluid, $16(3 \%)$ vascular access, and $11(2 \%)$ in a code scenario. Level 2 scans included musculoskeletal (40), including foreign body detection/removal (15 of 40) and ultrasound-assisted fracture reductions (14 of 40), shock-ACES (22), pneumothorax (20), hepatobiliary (18), urologic (16), DVT and other vascular indications (8), procedures such as nerve blocks (4), ultrasound in early pregnancy (3), and ophthalmic scans (3). Follow-up radiologic data were available for 279 scans. Of these, nine level 1 and five level 2 scans were discordant with criterion investigations; eight false positives and six false negatives. Sensitivity for both AAA screening scans and FAST was $96 \%$, while specificity was $100 \%$ and $93 \%$, respectively. Ultrasound-guided vascular access was $100 \%$ successful. Noteworthy level 2 applications were gallbladder, renal, and pneumothorax scans, which were all performed with $100 \%$ sensitivity and specificity. Conclusion: Use of ultrasound by emergency physicians was commonplace, with ultrasound being used on a daily basis. The vast majority of scans were for level 1 applications, and follow-up revealed that they were sensitive and specific for their indications. Level 2 applications were relatively infrequent but were similarly sensitive and specific. Keywords: health records review, sensitivity/specificity, ultrasound

Ballantine, Mike

mballantine2011@meds.uwo.ca

COMPUTED tomography head imaging in a rural emergency department for patients with traumatic head injury
Mike Ballantine, Parham Davoudpour, Munsif Bhimani, W. Ken Milne; Schulich School of Medicine and Dentistry, The University of Western Ontario, London, ON

Introduction: Head injury is a common occurrence, accounting for more than 8 million visits annually to North American emergency departments (ED). Our study sought to examine the rate of computed tomography $(\mathrm{CT})$ head imaging performed in patients presenting to a rural ED. Methods: A retrospective chart review was performed to determine the frequency of $\mathrm{CT}$ head in patients presenting to this rural ED with head injury. Additionally, total length of stay (LOS) in the department was investigated. Comparison was made to CT head rates seen in other studies. Results: A total of 102 cases of patients with traumatic head injury were reviewed. CT head was performed in $11 \%$ of patients. Positive CT head was seen in $2.9 \%$ of patients. Rates of CT head were significantly lower than in comparison studies. LOS of less than 6 hours was seen in $84 \%$ of patients. Conclusions: In this rural $\mathrm{ED}$, significantly fewer CT head are being ordered for patients with head injury compared to quoted averages. The vast majority of patients have a total LOS of less than 6 hours when presenting with head injury. Additional research can attempt to replicate these results in other rural EDs. Keywords: head trauma, neuroimaging, rural EM

Ellis, Paul

paul.ellis@uhn.on.ca

OPERATIONALIZING evidence-based quality of care indicators for Canadian emergency departments

Ellis PD and Ingram SA; University Health Network, Toronto, ON

Introduction: In March 2010, the ICES Investigative Report published Development of a Consensus on Evidence-Based Quality of Care Indicators for Canadian Emergency Departments. A set of 48 indicators were identified across nine clinical and operational categories. To date, there have been no published reports on standardize procedures to capture this data. We have done so for the four indicators in the Sepsis/Infection category. Methods: Patients presenting to the University Health Network's Emergency Department from April 1, 2009, to March 31, 2010, with a diagnosis of bacterial meningitis, severe sepsis, and septic shock were identified using ICD-10 codes. A chart review was then performed to capture the time to antibiotic administration for bacterial meningitis. Also captured was the percentage of patients with severe sepsis and septic shock receiving broad-spectrum antibiotics under 4 hours, surviving to discharge, and who were monitored for lactate clearance. Results: During the study time period, 16 patients with bacterial meningitis were identified with a median arrival to antibiotic time of 3.9 hours (1.4-12.5 hours). Twelve patients were identified with severe sepsis or septic shock. All patients received broad-spectrum antibiotics, and 75\% got them under 4 hours of arrival to the ER. Sixty-seven percent of the severe sepsis and septic shock patients survived to discharge, and the same percentage were monitored for lactate clearance. Conclusions: The University Health Network is a quaternary care teaching hospital with two emergency departments and about 80,000 ED visits per year. This was our first attempt at standardizing the collection of these data so that we can monitor ourselves annually and compare our data with similar hospitals across Canada. Keywords: quality of care, sepsis, time to treatment

McLeod, Shelley L

shelley.mcleod@1hsc.on.ca

EMERGENCY department disposition of sport-related acute mild traumatic brain injury

Ferguson A, McLeod SL, Millard W

Division of Emergency Medicine, Faculty of Medicine, The University of Western Ontario, London, ON 
Introduction: Acute mild traumatic brain injury (concussion/ATBI) is a common emergency department (ED) presentation with evidence-based recommendations regarding its management and disposition, including a graduated return to activity and close follow-up. The objective of this study was to determine if patients presenting to a tertiary care ED with a sports-related concussion were being managed according to these current practice guidelines. Methods: A retrospective medical record review was conducted at an academic tertiary care centre (annual census 65,000). Consecutive patients presenting to the ED over a 1-year period with a diagnosis of sports-related concussion had their patient records reviewed by trained research personnel, and data regarding the ED management and disposition were collected using a standardized data abstraction tool. Patients were excluded if they were younger than 10 years old or greater than 30 years old, were intoxicated, were involved in motor vehicle collision, or were not diagnosed with concussion. Results: Of the 62 charts reviewed, $40(64.5 \%)$ of the charts reviewed met the criteria for study inclusion. In $33(82 \%)$ cases, the patient was not asked about previous head injuries. Neuropsychological testing was done in only one $(2.5 \%)$ case. Discharge instructions were solely based on the head injury patient handout in $8(20 \%)$ cases. In $24(60 \%)$ cases, no follow-up was planned for the patient, and in $22(55 \%)$ cases reviewed, there was neither followup nor correct return to play guidelines documented. Conclusion: While practice guidelines exist to help guide the clinical decisions and ED disposition of concussed patients, it appears that these guidelines are not consistently followed. Improvements in the management of these patients should be implemented in order to improve patient care. Keywords: guideline compliance, health records review, traumatic brain injury

Lang, Eddy S

eddy.lang@albertahealthservices.ca

NATIONAL recommendations for prehospital transport of trauma patients developed using the GRADE methodology for evidence-based guidelines: air versus ground

Lang E, Brown K, Weik T, Spaite D, Falck-Ytter Y, Todd K, Dayan P, Gausch-Hill M, Oliver Z, Wright J, Sasson C, Sahni R, Hirshon J, Gotschall C, Dawson D, Howse D, Alcorta R, Hunt R; University of Calgary, Calgary, AB

Introduction: Decisions about helicopter transport (HEMS) of trauma patients has been driven by political, regulatory, and financial pressures and often not well informed by research assessing risks, benefits, and costs. We developed a practice guideline that provides directives for the optimal mode of transport using a well-established evidencebased guideline (EBG) framework.Methods: A multidisciplinary panel was recruited consisting of experts in trauma, EBG development, and EMS outcomes research. The panel used the Grading of Recommendations Assessment, Development, and Evaluation (GRADE) methodology to guide question formulation, evidence retrieval, and appraisal/synthesis and formulate recommendations. Panel members received GRADE training and conducted comprehensive literature reviews supported by health information specialists. Evidence profiles were developed around specific clinical questions, and graded recommendations were drafted. All graded recommendations (strong or weak), with descriptors of evidence quality (high, moderate, low, very low), were incorporated into an algorithm. GRADE permits the formulation of strong recommendations in the setting of weak evidence when a clear distinction between harms and benefits exists. A meeting was convened to review/endorse all materials and achieve consensus on recommendations. Results: Four strong and one weak recommendation emerged from the process, all supported only by low- or very lowquality evidence. Guidance was developed for mode of transport as a function of time savings between HEMS and ground (GEMS), use of online medical control, and considerations for local adaptation. The CDC National Triage Guideline was incorporated into the protocol. Conclusion: We successfully created a guideline and protocol for assigning mode of transport for trauma victims, developed through a validated EBG development process. Future research should define approaches for implementation of the guideline as well as the impact of the protocol on metrics of safety, outcomes, and cost. Keywords: clinical practice guideline, GRADE, helicopter EMS

Harris, Devin R

devinh@shaw.ca

\section{THREE-YEAR experience of an emergency department observa-} tion unit

Cheng L, Harris DR, Grafstein E, Innes G, Procter KA, Stenstrom R, Scheuermeyer F; Department of Emergency Medicine, St. Paul's Hospital; Department of Emergency Medicine, University of British Columbia, Vancouver, BC

Introduction: The St. Paul's Hospital Diagnosis and Treatment Unit (DTU) was modeled after similar observation units in the United States. This unit was intended to provide aggressive diagnosis and treatment of preidentified patient populations (eg, undifferentiated chest pain, asthma, etc.) while providing 24-hour discharge capability. In contrast to traditional "short-stay" admissions, through real-time monitoring and rapid, targeted access to hospital-based diagnostic testing and treatment, the intention was to shorten length of stay (LOS) without compromising quality of care. Methods: The DTU is a fourbed unit, opened in the beginning of 2006. Physicians enter orders and make DTU admission requests using a Computerized Physician Order Entry (CPOE) program. Demographic data, length of stay, medication administration, and diagnostic testing data are collected through the CPOE program. Results: Since April 1, 2007, to March 2010, 8,123 patients have been managed through the DTU. The five most common diagnoses admitted to the DTU were chest pain NYD (22.5\%); abdominal pain NYD (4.7\%); alcohol intoxication (3.7\%); pneumonia $(3.1 \%)$; and diarrhea/gastroenteritis $(2.9 \%)$. Average DTU volume per 24 hours was 6.3 patients in the four-bed unit. Average total ED LOS for DTU patients was 12.8 hours, with an average of 8.4 hours in the DTU. Admission rates for DTU patients were $11.0 \%$ in $07 / 08,10.9 \%$ in $08 / 09$, and $15.4 \%$ in $09 / 10$. Admission rates for short-stay (1 to 3 days) medical inpatients at St. Paul's Hospital declined during this time period, in comparison to other hospitals in the region $(p=.015 ; F$ statistic $=4.35[d f=2]$; repeated measures ANOVA). Conclusions: From this descriptive study, a DTU offers a safe and effective alternative to short-stay admissions. A more detailed analysis is necessary to determine avoided admissions and cost avoidance. Keywords: administrative database, emergency crowding, observations units

chan, chun man aedqeh@gmail.com

A PICTORIAL essay of emergent ultrasound of gastrointestinal tract in acute abdomen

Chan Chun Man, Chu Ka Man; Queen Elizabeth Hospital, Hong Kong

Introduction: Ultrasound is a noninvasive, nonionizing, first-line investigation for patients with acute abdomen. However, there is significant artifact arising from gas in the lumen of the gastrointestinal tract, which may lead the investigators to ignore the hollow viscera when performing abdominal ultrasound. Therefore, knowledge of sonographic findings of common emergent gastrointestinal tract pathologies is crucial to make the diagnosis accurately so that the patient's treatment will not be delayed. This presentation is to illustrate the sonographic imaging features of common emergent gastrointestinal tract pathologies. Methods: We review all urgent abdominal ultrasound examinations done between October 2008 and October 2010 in our centre. The disease spectrum and their salient imaging features will be discussed. Results: There are a number of cases of emergent gastrointestinal tract pathologies identified, including pediatric and adult 
groups. The pediatric group includes intussusception, acute appendicitis, mesenteric adenitis, inguinal hernia with intestinal obstruction, small bowel volvulus, small bowel hematoma secondary to HenochSchönlein purpura, hypertrophic pyloric stenosis, and intestinal duplication cyst. The adult group includes acute appendicitis, colonic diverticulitis, small bowel ischemia, and pneumoperitoneum. Their salient sonographic features and supplementary investigations, including radiograph and computed tomography, will be discussed. Conclusions: With the knowledge of sonographic findings of common emergent gastrointestinal tract pathologies, an accurate radiologic diagnosis can be made as early as possible. Keywords: acute abdomen, health records review, ultrasound

\section{Campbell, Samuel G}

emsgc@cdha.nshealth.ca

THE FACILITATION of care of low-acuity emergency department patients by advanced care paramedics

Campbell SG, MacKinley RP, Janes SE, Froese P, and Etsell G; Queen Elizabeth Health Sciences Centre, Halifax, NS

Introduction: A predicted shortage of both emergency physicians and emergency nurses suggests that alternative ways of safely providing emergency care are needed. Physician substitutes have been used with varying degrees of success. We describe the use of advanced care paramedics as physician extenders in the management of low-acuity emergency patients. Their duties include patient assessment, wound care and repair, casting and splinting, and medication administration. Methods: Eighteen months after initiating the practice, we reviewed the percentage of ED patients who were successfully streamed to this area and their length of stay (LOS) times (ED registration to discharge) relative to other ED patients. Results: Between July 1, 2009, and January 1, $2011,24,312$ patients of a total census of $93,354(26 \%)$ were seen in the area. LOS were 3.22 hours compared to 6.26 hours for patients not streamed to this area. The service was well received by patients and housestaff, and the hours of coverage has been increased. Conclusions: The number of patients whose ED care was facilitated by ACPs is greater than the census of most Nova Scotian emergency departments. This practice is associated with lower LOS than higher-acuity patients without using nursing resources and offers a viable alternative to traditional nursing or physician-centred emergency care. Keywords: advanced care paramedics, facilitation of care, low-acuity patients

\section{Thompson, Graham C}

graham.thompson@albertahealthservices.ca

OUTCOMES of children with suspected appendicitis in whom the appendix cannot be fully visualized by ultrasonography

Marshall Ross, Ellen Morrison, Ping Wei Chen, Walid Abou Reslan, Graham Boag, Robin Eccles, Graham Thompson; Alberta Health Services, University of Calgary, Calgary, $\mathrm{AB}$

Introduction: The diagnosis of appendicitis in children can be difficult, leading to an increased reliance on diagnostic imaging (DI) studies. Due to significant concerns regarding radiation exposure from computed tomography (CT), ultrasonography (U/S) has become an increasingly utilized resource. However, these DI exams often fail to fully visualize the appendix, leading to the inability to exclude appendicitis. The clinical outcome of these patients is unknown. Methods: A retrospective health record review of children aged 3-17 years presenting to a tertiary care pediatric emergency department (PED) with suspected appendicitis in whom the appendix could not be fully visualized. Primary outcome was PED disposition. Secondary outcomes included surgical consultation and intervention, use of further DI studies, and return PED visits. Results: Of $122 \mathrm{U} / \mathrm{S}$ performed for suspected appendicitis, the appendix could not be fully visualized in 71 (58.2\%). Admission to hospital occurred in $31(43.7 \%)$ of these chil- dren; an identical number were discharged home from the PED. The remaining children were asked to return for reassessment within 24 hours. Thirty-five $(49.3 \%)$ children received surgical consultation in the ED. Of the 31 children admitted, $13(41.9 \%)$ went directly to the operating room (OR) from the ED, while 18 (58.1\%) were observed. Ultimately, 23 (32.4\%) children with an incompletely visualized appendix underwent appendectomy. Nineteen $(26.8 \%)$ children received further DI studies (7 U/S, $12 \mathrm{CT})$. Eight $(11.3 \%)$ children discharged from the PED had an unscheduled return visit. Conclusions: Appendicitis cannot be fully excluded by $\mathrm{U} / \mathrm{S}$ in a significant number of children due to incomplete/nonvisualization of the appendix. Many of these children go on to have surgical consultation, admission, and, ultimately, an appendectomy. In lieu of significant delays related to obtaining DI studies, departmental policies and clinical pathways should consider using clinical criteria to determine high-risk children who would benefit from direct surgical consultation. Keywords: appendicitis, health records review, ultrasound

McLeod, Shelley L

shelley.mcleod@lhsc.on.ca

PREHOSPITAL medical directives: how often are they being applied when indicated? A retrospective review

Sereda A, Vandeyar T, McLeod S, Dukelow A, Lewell M, Davis M, Rodriguez S; Division of Emergency Medicine, Faculty of Medicine, The University of Western Ontario, London, ON

Introduction: Prehospital medical directives are used by emergency medical services (EMS) personnel to initiate medical treatment before patients arrive in the emergency department (ED). The objective of this study was to determine the proportion of patients transported by EMS who were eligible for medical directive care and the proportion of eligible patients who received directive-based care. Methods: A retrospective medical record review was conducted for adult ( $\geq 18$ years) patients transported to the ED via EMS over a 6month period. Paramedics' application of a prehospital medical directive was obtained from the ambulance call record (ACR) and classified according to the local 2005 "Primary Care Paramedic Medical Directives." The patient's chief complaint and vital signs were collected from the ACR using a standardized data extraction tool. Investigators used this information to determine whether patients meet the criteria for the application of the appropriate prehospital medical directive. Results: A total of 101 charts were reviewed. Eighteen (18\%) patients received care through a prehospital medical directive. Thirty-three $(33 \%)$ were treated using the cardiac chest pain protocol, 22 (22\%) using the SOB protocol, 39 (39\%) using the IV access protocol, and $5(5 \%)$ using the hypoglycemia protocol. It was determined that 3 patients, in addition to the 18 identified by paramedics, qualified for application of a prehospital medical directive. Paramedics correctly identified 18 of 21 (86\%) patients who qualified for the administration of a prehospital directive. No patients were incorrectly identified as requiring a prehospital medical directive. Conclusions: Paramedics are identifying patients who meet the criteria for prehospital medical directives with a high level of accuracy. Paramedics are not applying prehospital medical directives in patients who do not meet the required criteria for those medical directives. Keywords: health records review, prehospital medical directives, protocol compliance

Atkinson, Paul

Paul.Atkinson@HorizonNB.ca

FREQUENT users of the emergency department in southern New Brunswick: are they less likely to have access to a family physician?

Erin Palmer, Paul Atkinson; Saint John Regional Hospital, Saint John, NB 
Introduction: Emergency department overcrowding had become an important public health issue in Canada. Often lack of access to primary care is cited as a cause for overcrowding, despite the fact that there is little evidence to support this theory. We aimed to determine if having access to a primary care physician affects frequency of emergency department use in three emergency departments in southern New Brunswick. Methods: All 117,406 visits to the Saint John Regional Hospital (SJRH), St. Joseph's Hospital (SJH), and Sussex Health Centre (SHC) emergency departments in the year 2009 were selected using a central computerized database. Patients with listed family physicians were compared to those without listed family physicians in regard to number of visits to the emergency department within that 1-year period. Ordinal data were analyzed using chi-square test and interval data using Student $t$-test (GraphPad). Results: The highest number of visits for any individual patient at each site was 85 . Frequent attenders ( $\geq 4$ visits) accounted for $5.7 \%$, $5.8 \%$, and $15.8 \%$ of all patients, respectively. Such patients were significantly more likely to have a family physician $(p<.0001)$. The proportion of patients with no listed family physician was $63.4 \%$ for SJRH, $62.9 \%$ for SJH, and $10.6 \%$ for SHC. The mean number of visits for each site were 1.66, 1.67, 2.14 (family physician) and 1.47, $1.49,1.73$ (no family physician). The mean number of visits was significantly higher $(p<.0001)$ for patients with a family physician. Conclusion: Although the majority of patients attending urban centres (SJRH and SJH) were without a listed family physician, the frequency of attendance to each emergency department was higher for patients with listed family physicians at all sites. Keywords: administrative database, frequent ED users, primary care access

\section{Atkinson, Paul}

\section{Paul.Atkinson@HorizonNB.ca}

ULTRASOUND-guided pediatric forearm fracture reduction during sedation in the emergency department

Paul Atkinson, Khurram Iftikhar, Asif Naveed, Richard Kendall, Lee van Rensburg, Rajeev Madan; Saint John Regional Hospital, Saint John, NB; University of Cambridge, Cambridge, United Kingdom

Introduction: Displaced forearm fractures in children are a common emergency department (ED) presentation, and many are admitted for manipulation under general anesthetic. Ketamine is increasingly being used for sedation of patients undergoing painful procedures within the emergency department. Ultrasound can be used to detect fractures in children. It is easy to perform and yields good results. We describe the use of ultrasound to guide fracture reduction for manipulation in the emergency department. Methods: We describe a case series of five patients who recently attended with isolated forearm injuries. After appropriate sedation being administered, imaging was performed using a linear ultrasound transducer, placed longitudinally over the fracture site. The cortex of the bone was clearly visible as a bright white line. The fracture line and any displacement were identified. After manipulation, alignment was checked on ultrasound. If the position was deemed unacceptable, further attempts at reduction were made until satisfactory reduction was achieved before application of a cast, or it was determined that further attempts would require general anesthetic. Postreduction radiographs were obtained. Results: In our small case series, we demonstrate that ultrasound can be used to accurately assess manipulation of distal forearm fractures in the ED both as a positive and negative predictor of success. Conclusions: Applying this technique in the ED setting could potentially reduce the number of admissions for numerous children presenting with displaced forearm fractures. A further larger trial and economic analysis is needed to confirm the benefits of using ultrasound to assist in manipulation of forearm fractures. Keywords: case series, pediatric forearm fractures, ultrasound
McLeod, Shelley L

shelley.mcleod@1hsc.on.ca

THE SENSITIVITY of CT scanning in the diagnosis of spontaneous subarachnoid hemorrhage in patients presenting at various times after their headache onset

Loubani M, McLeod SL, Fuss J; Division of Emergency Medicine, Faculty of Medicine, The University of Western Ontario, London, ON

Introduction: Spontaneous subarachnoid hemorrhages (SSAHs) presenting to the emergency department (ED) pose a diagnostic challenge. Since CT scans (CT) of the head are inadequately sensitive to exclude SSAHs completely, physicians entertaining a diagnosis of SSAH often perform a lumbar puncture (LP) after a negative scan. The objective of this study was to evaluate the sensitivity of CT in the diagnosis of SSAH in patients presenting at various times after their headache onset. Methods: A retrospective medical record review was conducted to analyze CT scans performed to assess patients presenting with sudden headache. Patients who were febrile, had sustained trauma, had focal neurologic deficits, or had a known SSAH or where a negative CT was not followed by LP were excluded. Records included in the study were analyzed for vital data and the results of the CT scan and/or LP results. Results: A total of 142 medical records met the inclusion criteria. Of these, 17 had SSAHs found on CT scan alone $(3 \leq 6$ hours from headache onset; 3 between 6 and 12 hours; $3 \geq 24$ hours; and 8 where the time of onset was not documented), while 2 SSAHs were missed on initial CT and diagnosed on subsequent LP, both after $\geq 24$ hours from headache onset ( 1 at almost 48 hours and 1 at 10 days after onset). As such, CT was found to be $89.5 \%$ sensitive in detecting SSAH in all patients presenting with sudden headache and $100 \%$ sensitive in detecting SSAH in those presenting within 24 hours of the onset of their headache. Conclusions: Although CT continues to have inadequate sensitivity for detecting SSAHs in every patient presenting with a sudden-onset headache, this study suggests that patients presenting within 24 hours of their headache onset may potentially have a diagnosis of SSAH safely excluded with CT alone. Keywords: neuroimaging, sensitivity/specificity, subarachnoid hemmorhage

Chu, Jennifer L

jenniferchu15@gmail.com

USE of a social networking website for recruiting Canadian youth for medical research

Jennifer Chu, Carolyn Snider; University of Toronto, Toronto, ON

Introduction: The use of the Internet gained widespread popularity in the 1990s, and the next decade led to an explosion of social networking websites such as MySpace and Facebook. While these websites were initially marketed as a means for social use, the business sector quickly realized the potential of social networking sites. Facebook is one of the most popular websites today and has gained an enormous amount of users since its worldwide launch in 2006. Current reports show that over 11.5 million Canadians are Facebook users, including over $99 \%$ of Canadians aged 18-34 years. Facebook allows users to purchase advertising space, which displays banner advertisements on the website. Advertisers may target members based on age, gender, geographic location and to members of specific Facebook groups. Advertising on social networking sites for medical research is not widely utilized, and very little research has looked into the potential of these websites. Methods: A survey study was designed to evaluate the prevalence of PTSD in Canadian youth who were affected by violence. To find subjects from a wide population, participants were recruited from an advertisement on Facebook that targeted Canadian users aged 15 to 24 years. They were asked if they had been exposed to violence, and if they clicked on the advertisement, they were linked to an online survey. This secondary analysis is a descriptive study on the effectiveness of the Facebook campaign. Data on advertising parameters were 
abstracted from the website. Results: An interim analysis showed that in the first 3 months, the advertisement was displayed 6.74 million times, resulting in 1,161 clicks on the link to the survey $(0.017 \%)$. The overall cost worked out to $\$ 0.42$ per click or $\$ 0.07$ per 1,000 displays. Thirty-seven participants completed the survey. Conclusion: Facebook advertising is an inexpensive, easy-to-use method that allows for distribution to a very wide population. There are many potential uses for social networking in medical research. Keywords: posttraumatic stress disorder, social networking, subject recruitment

Chu, Jennifer L jenniferchu15@gmail.com

PREVALENCE of PTSD in Canadian youth exposed to violence Jennifer Chu, Carolyn Snider, University of Toronto, Toronto, ON

Introduction: An emergency department (ED) visit may be the only contact that young victims of violence have with the health care system. A study based in Toronto showed that almost $90 \%$ of youths injured by violence are discharged directly from the ED, and in many EDs, little mental health follow-up is provided. Victims of violence are at risk of developing posttraumatic stress disorder (PTSD), which can lead to devastating complications, including occupational impairment, substance abuse, depression, physical health impairment, and suicide.
It has also been established as an immediate risk factor for both committing violence and becoming a victim. Most of the literature linking youth violence and mental health is based on studies involving American urban populations. Virtually no literature has looked at the effects of youth violence on mental health in a Canadian setting. To evaluate the need for prevention programs in the $\mathrm{ED}$, it is necessary to determine the prevalence of the problem. Methods: This is an observational study using survey methodology. The survey integrates the PTSD Checklist (civilian version) questionnaire, a validated self-reporting tool used to diagnose PTSD. Canadian youth aged 15-24 who witnessed or experienced violence were recruited using an advertisement on the social networking site Facebook, which linked participants to the online survey. A sample size of 88 was calculated for an estimated prevalence of $35 \%$ (with $10 \%$ precision) using American literature. Results: An interim analysis demonstrated 21 eligible subjects after 2 months. The prevalence of PTSD was $42.9 \%$ (21.7-64.0). Factors that showed trends to an increased risk for PTSD were female gender, ethnic minority, and being a victim of violence $(p=.18, .12, .07)$. Conclusions: The interim analysis demonstrates comparable prevalence to the American literature thus far. Final analysis may show a statistically significant increase in risk for females, minorities, and victims once sample sizes are larger. Keywords: posttraumatic stress disorder, survey, violence 